\title{
Ecological Interface Design for a Water Monitoring Decision Aid
}

by

\author{
Kevin Kan
}

A thesis submitted in conformity with the requirements for the degree of Master in Applied Science

Graduate Department of Mechanical and Industrial Engineering University of Toronto

C Copyright by Kevin Kan (2012) 


\title{
Ecological Interface Design for a Water Monitoring Decision Aid
}

\author{
Kevin Kan \\ Master of Applied Science \\ Department of Mechanical and Industrial Engineering \\ University of Toronto \\ 2012
}

\section{Abstract}

In joint human-automation systems, operators must often supervise the automation and adapt their reliance on it based on judgments of its context-specific reliability. For this to occur, operators should trust the automation appropriately. In the design of a water monitoring decision aid's display, Ecological Interface Design was used to satisfy design guidelines for supporting appropriate trust. Design focused upon a visualization that made the aid's use of the DempsterShafer theory directly perceptible. The display was evaluated using a signal detection theorybased approach that measured reliance on automation. Results indicated that the ecological display yielded less appropriate reliance and poorer performance than a conventional display for a highly reliable decision aid. However, the experimental task prevented participants from adapting to the aid's context-specific reliabilities, reducing the validity of the findings. A subsequent study is proposed to further study the effects of ecological displays on automation reliance. 


\section{Acknowledgements}

I thank my supervisor, Dr. Greg A. Jamieson, for his mentorship in the completion of this thesis. I am especially grateful for his introduction to human factors during my undergraduate education. I owe much of my interest in this field to his teaching and guidance.

I feel fortunate to have been a member of the Cognitive Engineering Laboratory. My colleagues' perspectives on my research were insightful and encouraging. Even beyond research matters, their company and conversations will be warmly remembered.

I thank my committee members, Dr. Justin Hollands and Dr. Paul Milgram, for their time and interest in reviewing my work. Their feedback is very much appreciated.

I also thank A.U.G. Signals for being a project partner and for taking the time to explain their technologies to me. Their detailed explanations and patience with my questions were crucial to this research.

Finally, I am indebted to my family for their continued support and commitment to me. 


\section{Table of Contents}

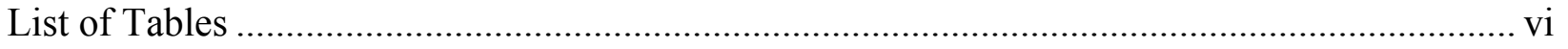

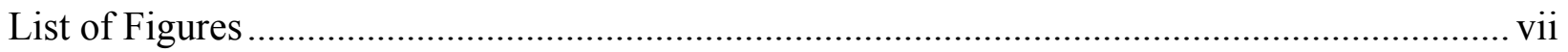

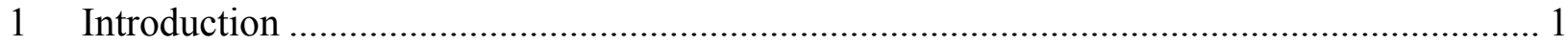

1.1 The Intelligent Drinking Water Monitoring System ........................................... 1

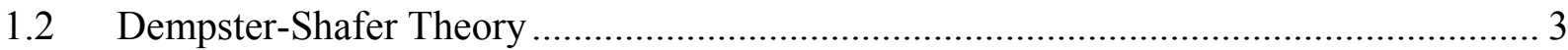

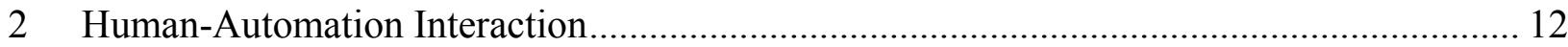

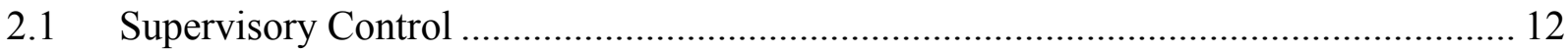

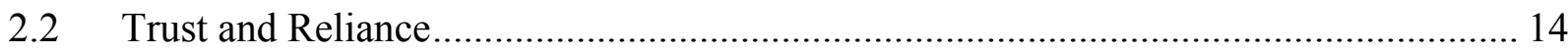

2.3 Implications for Decision Aids ....................................................................... 16

2.4 Modelling Human-Automation Interaction......................................................... 17

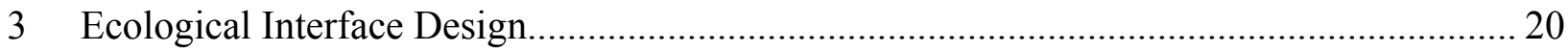

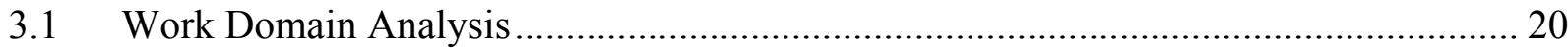

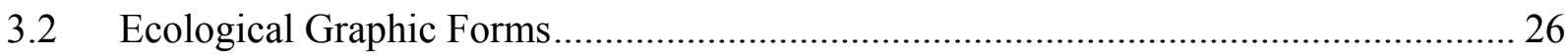

4 Work Domain Analysis for the Intelligent Drinking Water Monitoring System ................ 28

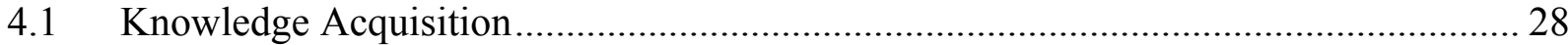

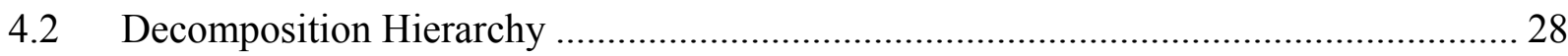

$4.3 \quad$ Abstraction-Decomposition Space .................................................................... 29

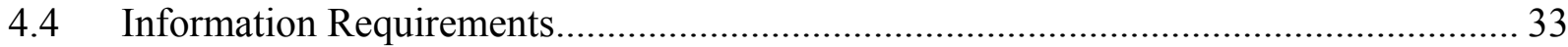

5 Display Design for Dempster's Rule of Combination................................................... 38

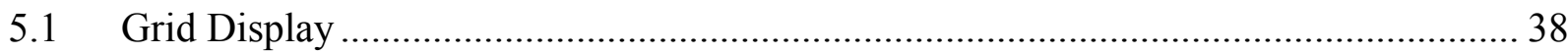

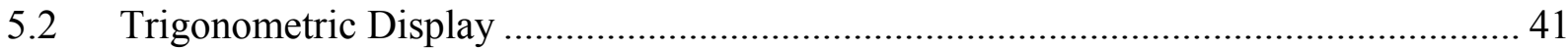

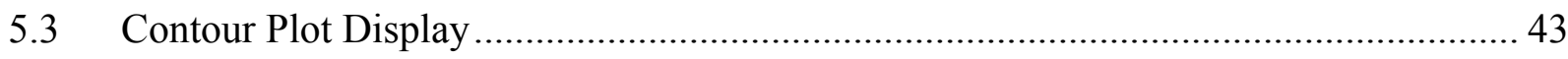

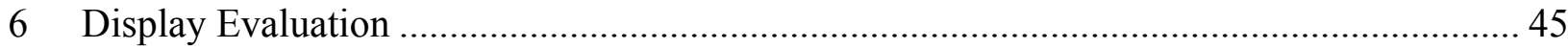

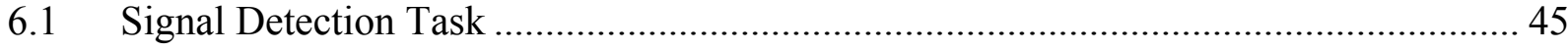

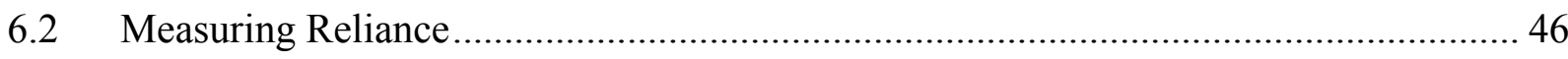

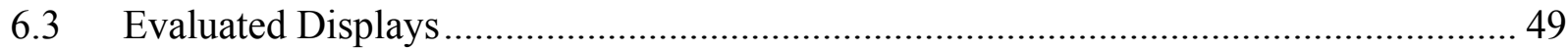

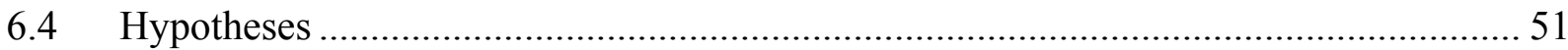

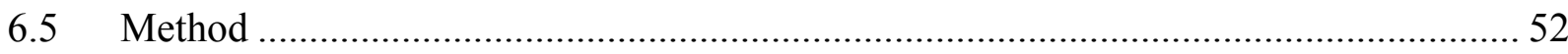

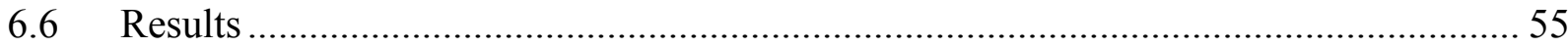

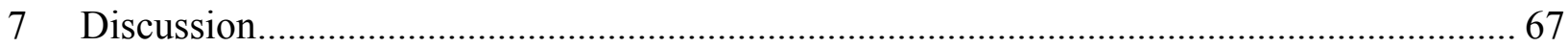




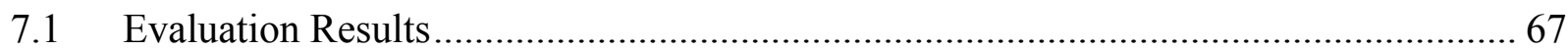

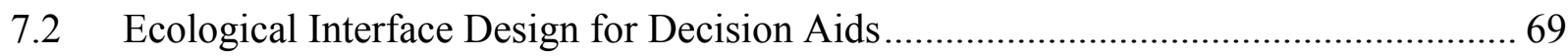

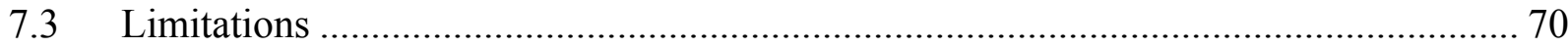

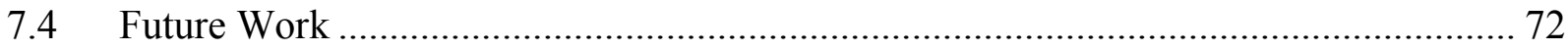

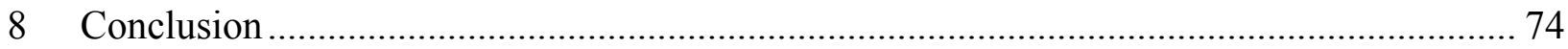

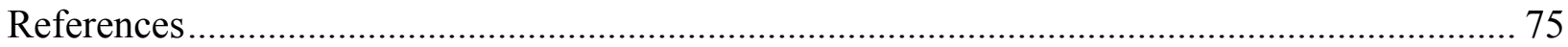

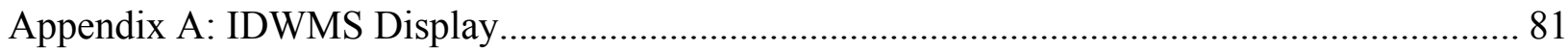

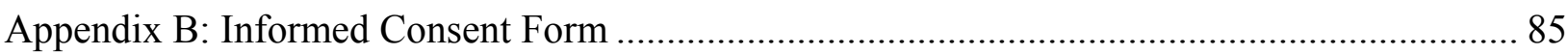

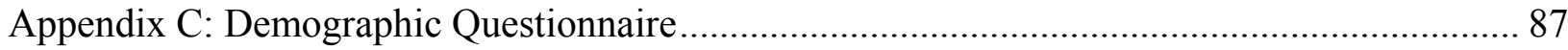

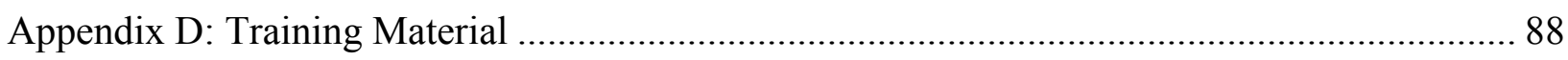

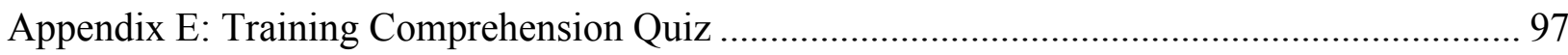

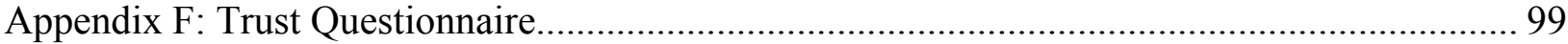




\section{List of Tables}

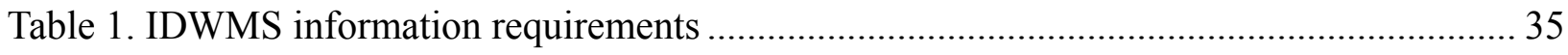

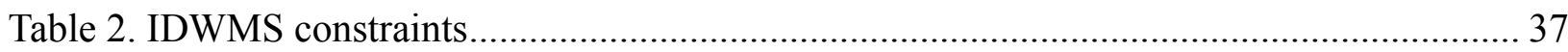




\section{List of Figures}

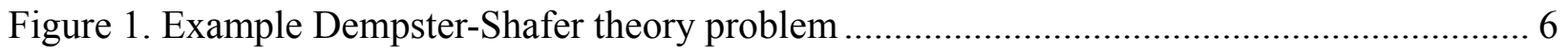

Figure 2. Example Dempster-Shafer theory problem with a high degree of conflict................... 7

Figure 3. IDWMS implementation of Dempster's rule of combination...................................... 8

Figure 4. IDWMS-based example of a misleading Dempster's rule result ............................. 10

Figure 5. Definitions of supervisory control (from Sheridan, 2006) ..................................... 13

Figure 6. Types and levels of automation (from Parasuraman et al., 2000) ............................. 14

Figure 7. Characterization of automated decision aids using types and levels of automation

(adapted from Parasuraman et al., 2000) ................................................................................ 17

Figure 8. An empty abstraction-decomposition space (ADS; Vicente, 1999) .......................... 21

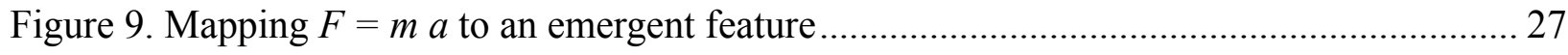

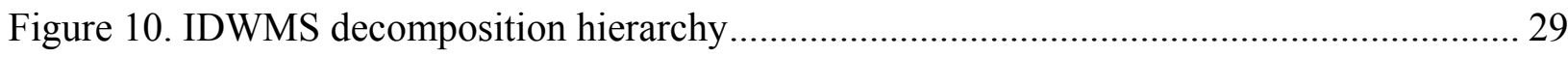

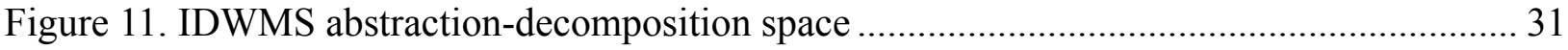

Figure 12. IDWMS abstraction-decomposition space (continued) ....................................... 32

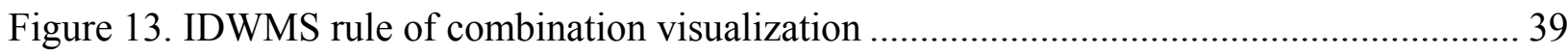

Figure 14. Visualization of Bayes' law (from Burns, 2004) .............................................. 40

Figure 15. Trigonometric mapping for a multiplicative relationship.................................... 41

Figure 16. IDWMS trigonometric display for Dempster's rule of combination ........................ 42

Figure 17. IDWMS contour plot for Dempster's rule ........................................................ 43

Figure 18. Three-dimensional plot of Dempster's rule ..................................................... 44

Figure 19. Experimental task screen with integrated display .............................................. 50

Figure 20. Experimental task screen with separated display ............................................. 51

Figure 21. Experimental task screen in the no-aid (manual) condition .................................... 53

Figure 22. Effects of aid reliability and display on detection sensitivity, $\mathrm{d}^{\prime}$............................. 56

Figure 23. Effects of aid reliability and display on miss rate .............................................. 57

Figure 24. Effects of aid reliability and display on false alarm rate ..................................... 58

Figure 25. Effects of aid reliability and display on response time........................................ 59

Figure 26. Effects of aid feedback and display on $\ln \beta$ differences for the no-aid $-80 \%$ reliability

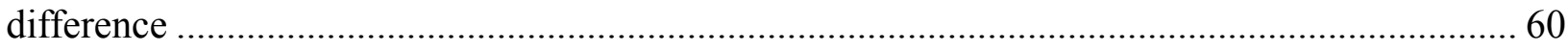

Figure 27. Effects of aid feedback and display on $\ln \beta$ differences for the $80 \%-94 \%$ reliability difference 
Figure 28. One-sample two-tailed $t$-tests comparing observed and effective $\ln \beta$ differences for signal feedback.

Figure 29. One-sample two-tailed $t$-tests comparing observed and effective $\ln \beta$ differences for signal feedback.

Figure 30. Effects of aid feedback and display on $\mathrm{C}$ differences for the no-aid $-80 \%$ reliability difference 63

Figure 31. Effects of aid feedback and display on C differences for the $80 \%-94 \%$ reliability difference 64

Figure 32. Effects of aid result and reliability on error rate for the separated display ..................65 65

Figure 33. Effects of aid result and reliability on error rate for the integrated display.................. 65

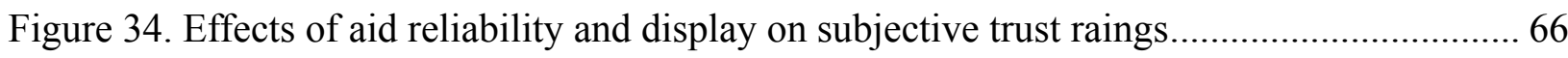

Figure 35. Interactive visualization for Bayes' law (from Tsai et al., 2011) ................................. 69

Figure 36. Conflicting codes in the integrated display used for evaluation................................... 71 


\section{Introduction}

Given society's reliance on water distribution networks, there is growing concern over their vulnerability to both man-made and natural contamination events (Clark, Hakim, \& Ostfield, 2011). Water quality may be compromised by such factors as aging infrastructure, natural disasters, and terrorist attacks. Despite the importance of distribution networks, current methods of contaminant detection are unable to provide timely detection of such events (Clark et al., 2011). Surveillance of these networks is a significant challenge given that they are large, distributed, and interconnected with other infrastructure. Control room operators can rely upon always-available chlorine sensor readings as an indicator of possible intrusions, but this method can neither identify nor quantify contaminants. To do so requires water laboratory testing of manually collected water samples, a process that is typically performed infrequently and requires days to complete. Such methods are inadequate, as contamination events are characterized as being short but intense (Clark et al., 2011). To complement this sampling approach and alleviate its shortcomings, online water monitoring systems are being developed to serve as early warning and detection systems.

\subsection{The Intelligent Drinking Water Monitoring System}

The Intelligent Drinking Water Monitoring System (IDWMS) is a decision aid that helps operators detect water anomalies in drinking water distribution networks. The system uses sensor sites installed through a distribution network to sample water at regular intervals. Each sensor site contains an array of five conventional sensors that measure total chlorine, $\mathrm{pH}$, conductivity, oxidation reduction potential, and turbidity. In addition, each sensor site is equipped with a spectrometer that acquires each sample's ultraviolet-visible absorption spectra. The acquired data is transmitted to a central processing unit for data analysis so that the quality of the sampled water can be inferred.

For each conventional parameter, the aid compares the sensor readings to threshold values configured by control room operators. Based on the readings' distance from these limits, the aid computes its confidence that the sampled water is normal, as well as its confidence that the sampled water is abnormal. As a separate analysis, the aid compares the sampled water's spectrum to a baseline spectrum for normal water to determine another pair of normal-abnormal 
confidence values. To produce a final result, the aid fuses the two pairs of confidence values to produce a final pair of normal-abnormal confidence values. The IDWMS also compares the sample's spectrum to a database of contaminants' reference spectra. For each reference, the system computes its confidence in the contaminant's existence, as well as the most likely concentration.

While the IDWMS promises earlier and more sensitive detection of water anomalies, it is not perfect. It will inevitably be incomplete in its data acquisition and analyses given sensor limitations and the inherent uncertainty of statistical inference. For example, the reliability of sensor readings is subject to environmental conditions: the presence of bubbles will interfere with the measurement of certain parameters, and certain parameters are correlated with temperature. In addition, the spectroscopy process can only identify contaminants that have had their spectra stored in the IDWMS database. Storing spectra is a manual process, as is the seasonal calibration of operating thresholds for the conventional water quality parameters. Furthermore, the inference process employed by the IDWMS can provide misleading results in certain situations (described in section 1.2).

These limitations all create uncertainty about the true health of the water in the distribution network. It is therefore important that operators rely upon the decision aid appropriately when assessing water quality. Results from the IDWMS need to be considered in the context of other data, such as customer feedback and government reports, as well as readings from the distribution network's supervisory control and data acquisition (SCADA) system. Research in human-automation interaction provides an understanding of how operators interact with automation, as well as how they might be supported in appropriately relying upon the IDWMS, such that water quality monitoring performance can be improved.

In many joint human-automation systems, operators can be considered supervisors to the subordinate automation: they must verify that the automation functions as intended and intervene when it does not (Sheridan, 2006). That is, operators must adapt their reliance upon the automation in a context-specific manner. However, it is impractical for operators to fully understand complex automation and when such uncertainty exists, their trust in the automation guides reliance (Lee \& See, 2004). Trust is the operator's attitude that the automation will help achieve his goals when he relies upon it in uncertain situations (Lee \& See, 2004). 
Displays can be used to foster appropriate trust and guidelines have been proposed for their design (Lee \& See, 2004). However, no definitive approach to satisfying these guidelines has been identified. To this end, the current thesis investigates the applicability of Ecological Interface Design (Burns \& Hajdukiewicz, 2004; Vicente \& Rasmussen, 1990; Vicente \& Rasmussen, 1992) to identifying information about automation capability and displaying it in a manner that is directly perceptible to operators. The design goal is to improve joint humanautomation decision-making performance by supporting appropriate trust and reliance on the automation.

\subsection{Dempster-Shafer Theory}

For each of the five conventional water quality parameters, the IDWMS compares sensor readings to stored upper and lower threshold values. Based on this comparison, the "confidence" that sampled water is normal is calculated. Subtracting this normal confidence from $100 \%$ yields the confidence that the water is abnormal. For the spectroscopic analysis, an additional pair of normal-abnormal confidence values is calculated by comparing the sample's spectrum to a previously stored reference spectrum of normal water. To obtain final confidence values, the IDWMS fuses these six pairs of confidence values using the Dempster-Shafer theory and Dempster's rule of combination (Shafer, 1976). A basic introduction to this mathematical theory of evidence is presented to give an understanding of the IDWMS sensor fusion process.

In the Dempster-Shafer theory, a piece of evidence supports propositions. A proposition can be a single event, or it can be a set of events. A piece of evidence, or source of information, has different degrees of belief in different propositions. For example, Doctor 1 may believe in four propositions when diagnosing a patient:

1. The patient has condition $\mathrm{X}$, with a probability of 0.30 .

2. The patient has condition $\mathrm{Y}$, with a probability of 0.10 .

3. The patient has condition $\mathrm{X}$ or condition $\mathrm{Y}$, with a probability of 0.20 .

4. The patient has condition $\mathrm{X}$ or condition $\mathrm{Z}$ with a probability of 0.40 .

The degree of belief in each proposition is called a mass assignment. In the Dempster-Shafer theory notation, the degree of belief in some proposition $A$ is represented by the term $m(A)$. 
Together, these four example mass assignments form the mass assignment function for Doctor 1. A piece of evidence's mass assignment function must sum to 1 .

The Dempster-Shafer theory allows multiple pieces of evidence to be combined. For example, Doctor 2 may have the following mass assignment function:

1. The patient has condition $\mathrm{Y}$, with a probability of 0.50 .

2. The patient has condition $\mathrm{X}$ or condition $\mathrm{Y}$, with a probability of 0.30 .

3. The patient has condition $\mathrm{Z}$, with a probability of 0.20 .

Both doctors' mass assignment functions can be combined to determine the joint belief in a proposition using Dempster's rule of combination:

$$
\begin{gathered}
m_{D 1, D 2}(A)=\frac{1}{1-K} \sum_{B \cap C=A \neq \emptyset} m_{D 1}(B) m_{D 2}(C) \\
\text { where } K=\sum_{B \cap C=\varnothing} m_{D 1}(B) m_{D 2}(C)
\end{gathered}
$$

The $m_{D 1}$ and $m_{D 2}$ terms represent the mass assignments from Doctor 1 and Doctor 2, respectively. The variables $A, B$, and $C$ are propositions. $A$ is the proposition for which the joint belief will be calculated. For this example, proposition $A$ will be that the patient has condition Y. $B$ can represent any of the propositions for which Doctor 1 has a degree of belief. Similarly, $C$ can represent any of the propositions for which Doctor 2 has a degree of belief.

To calculate the joint belief in proposition $A, m_{D 1, D 2}(A)$, the following sum is first calculated:

$$
\sum_{B \cap C=A \neq \emptyset} m_{D 1}(B) m_{D 2}(C)
$$

Because proposition $A$ is that the patient has condition $\mathrm{Y}$, the condition $A \neq \emptyset$ is satisfied. This condition requires that proposition $A$ contains at least one event and is thus not the empty set, $\varnothing$. Next, every pair of Doctor 1's and Doctor 2's propositions is taken. For each pair, the intersection is determined; every pair of propositions must belong to one of three cases. The mass assignments for each pair of propositions belonging to case 1 are multiplied and summed. 
Case 1 . The intersection of propositions $B$ and $C$ is exactly equal to proposition $A$. For example, both Doctor 1 and Doctor 2 believe that the patient has condition Y. Another example is if Doctor 1 believes that the patient has condition Y or condition $\mathrm{X}$, and Doctor 2 believes that the patient has condition $\mathrm{Y}$.

Case 2. The intersection of propositions $B$ and $C$ equals the empty set, $\varnothing$. For example, Doctor 1 believes that the patient has condition X, while Doctor 2 believes that the patient has condition $\mathrm{Y}$.

Case 3. The intersection of both propositions $B$ and $C$ is not exactly equal to proposition $\mathrm{A}$, nor is it the empty set, $\varnothing$. For example, both Doctor 1 and Doctor 2 believe that the patient has condition X. Another example is if both Doctor 1 and Doctor 2 believe that the patient has condition $\mathrm{X}$ or condition $\mathrm{Y}$.

To calculate the second sum, $K$, the mass assignments for each pair of propositions belonging to case 2 are multiplied and summed. To obtain the joint belief in proposition $A$, the sum for case 1 (the degree to which both doctors agree that the patient has condition $\mathrm{X}$ ) is divided by $1-K$ (where $K$ represents the degree to which both doctors disagree). To illustrate this example, Shafer's (1976) visualization for the rule of combination is used (Figure 1). The vertical dimension corresponds to Doctor 1's mass assignment function; the total length is divided according to the mass assignments for each of Doctor 1's propositions. Similarly, the horizontal dimension corresponds to Doctor 2's mass assignment function.

Thus, case 1 is represented by the dark gray areas (areas A, D, and E) in Figure 1. Summing these areas yields the term. Case 3 is represented by the uncoloured areas (areas C, F, G, J and L) in Figure 1. Therefore, the joint belief that the patient has condition $\mathrm{Y}$ is:

$$
m_{D 1, D 2}(\text { condition } Y)=(\mathrm{A}+\mathrm{D}+\mathrm{E}) /[1-(\mathrm{C}+\mathrm{F}+\mathrm{G}+\mathrm{J}+\mathrm{L})]
$$

Visually, the solution is the proportion of dark gray area to the total shaded area. 


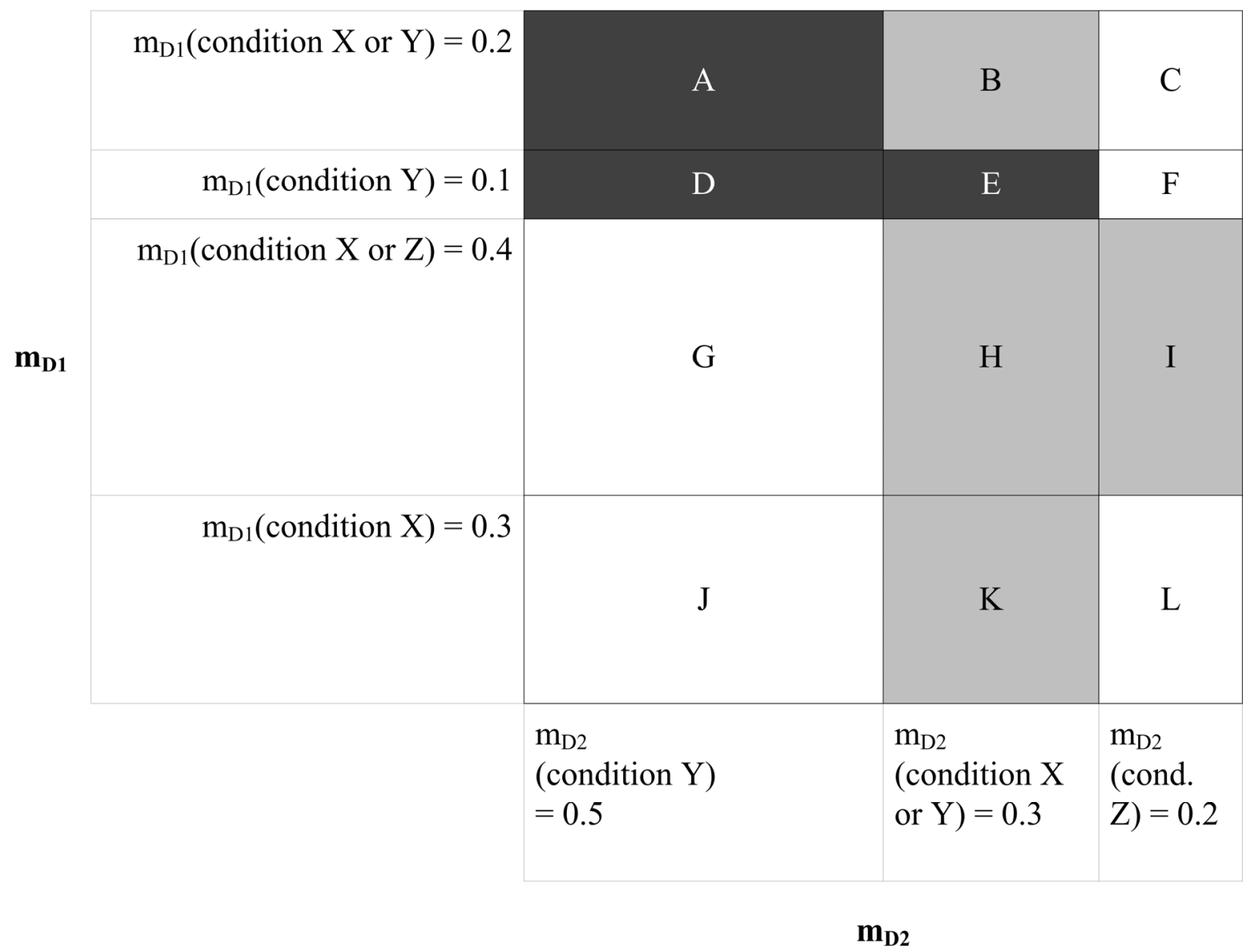

Figure 1. Example Dempster-Shafer theory problem

The term $1-K$ is called the normalization factor, and its use in the denominator of Dempster's rule causes the rule to ignore any conflicting evidence. The normalization factor's significance can be seen in situations with highly conflicting evidence. For example, Doctor 1 and Doctor 2 might completely disagree about whether the patient has condition $\mathrm{X}$ or $\mathrm{Y}$, but might agree that there is a small probability that the patient has condition Z (Figure 2). With such mass assignment functions, Dempster's rule of combination yields an unintuitive result: there is complete belief that the patient has condition $\mathrm{Z}$. 


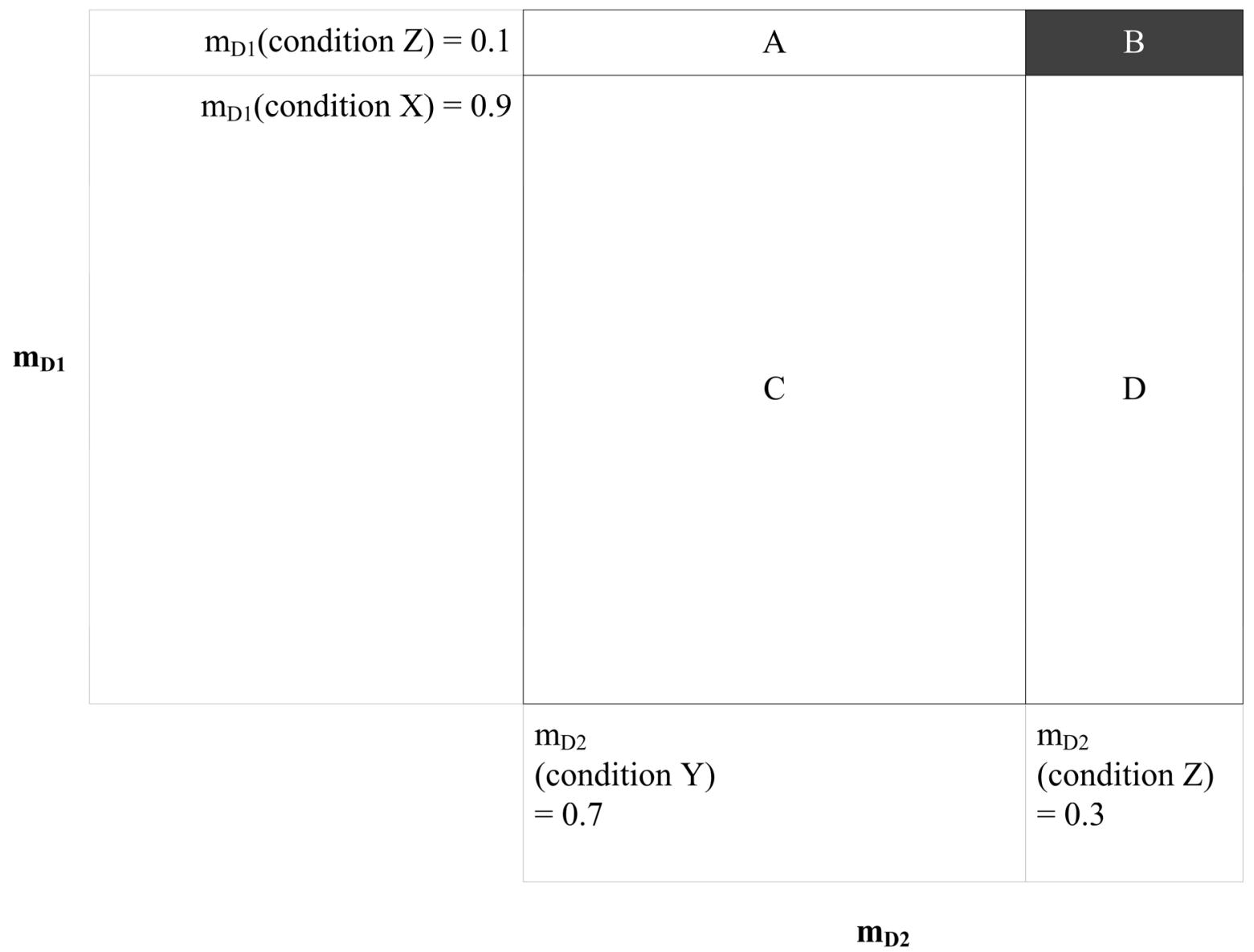

Figure 2. Example Dempster-Shafer theory problem with a high degree of conflict

\subsubsection{IDWMS Implementation of Dempster's Rule}

In the case of the IDWMS, two mutually-exclusive propositions are considered for a given water sample: the sample is normal and the sample is abnormal (Figure 3). As a result, Dempster's rule can be simplified. The two sources of information are the conventional analysis $\left(m_{\text {conv }}\right)$ and the spectroscopic analysis $\left(m_{\text {spec }}\right)$ performed by the aid. Thus, the IDWMS implementation of Dempster's rule is (assuming the proposition of interest is normal water): 


$$
\begin{gathered}
m_{\text {spec,conv }}(\text { Norm })=\frac{1}{1-K} \sum_{B \cap C=\text { Norm } \neq \varnothing} m_{\text {spec }}(B) m_{\text {conv }}(C) \\
\text { where } K=\sum_{B \cap C=\varnothing} m_{\text {spec }}(B) m_{\text {conv }}(C)
\end{gathered}
$$

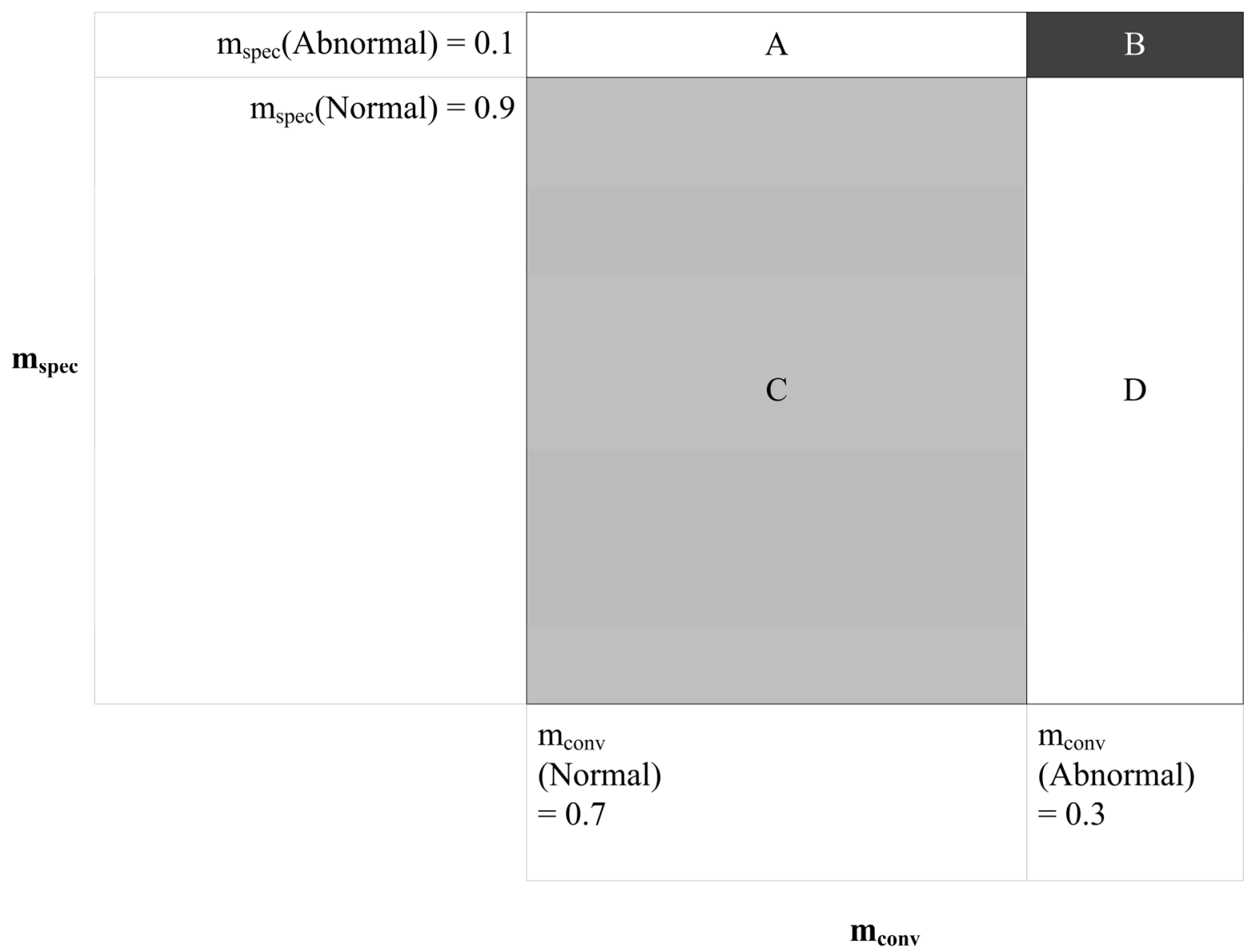

Figure 3. IDWMS implementation of Dempster's rule of combination

For the term $\sum_{B \cap C=N o r m \neq \varnothing} m_{\text {spec }}(B) m_{\text {conv }}(C)$, the only intersection that satisfies $B \cap C=$ Norm $\neq \varnothing$ is the normal water proposition for both analyses. This is represented by area $\mathrm{C}$ in Figure 3. For $K=\sum_{B \cap C=\varnothing} m_{\text {spec }}(B) m_{\text {conv }}(C)$, the only intersections that satisfy $B \cap C=\emptyset$ are represented by area A and area D. Furthermore, the IDWMS only considers the normal and abnormal water propositions, and the two mass assignments must sum to 1 for any source of information. Thus, Dempster's rule can be simplified as follows: 


$$
\begin{aligned}
& m_{\text {spec,conv }}(\text { Norm })=\frac{1}{1-K} \sum_{B \cap C=\text { Norm } \neq \varnothing} m_{\text {spec }}(B) m_{\text {conv }}(C) \\
& =\frac{m_{\text {spec }}(\text { Norm }) m_{\text {conv }}(\text { Norm })}{1-\left[m_{\text {spec }}(\text { Abnorm }) m_{\text {conv }}(\text { Norm })+m_{\text {spec }}(\text { Norm }) m_{\text {conv }}(\text { Abnorm })\right]} \\
& =\frac{m_{\text {spec }}(\text { Norm }) m_{\text {conv }}(\text { Norm })}{m_{\text {spec }}(\text { Norm }) m_{\text {conv }}(\text { Norm })+m_{\text {spec }}(\text { Abnorm }) m_{\text {conv }}(\text { Abnorm })}
\end{aligned}
$$

As an IDWMS-based example of a misleading result produced by Dempster's rule, the spectroscopic analysis is much more sensitive to contaminants than the conventional analysis, especially at low contaminant concentration. Typically, only a subset of the conventional water quality parameters will react to a contaminant, which frequently results in a high (and sometimes complete) conventional belief in normal water. This can produce the mass assignments in Figure 4. The resulting joint mass assignment function will be misleading; it will indicate a complete belief in normal water (area C), since the normalization factor will ignore the disagreement represented by area A. 


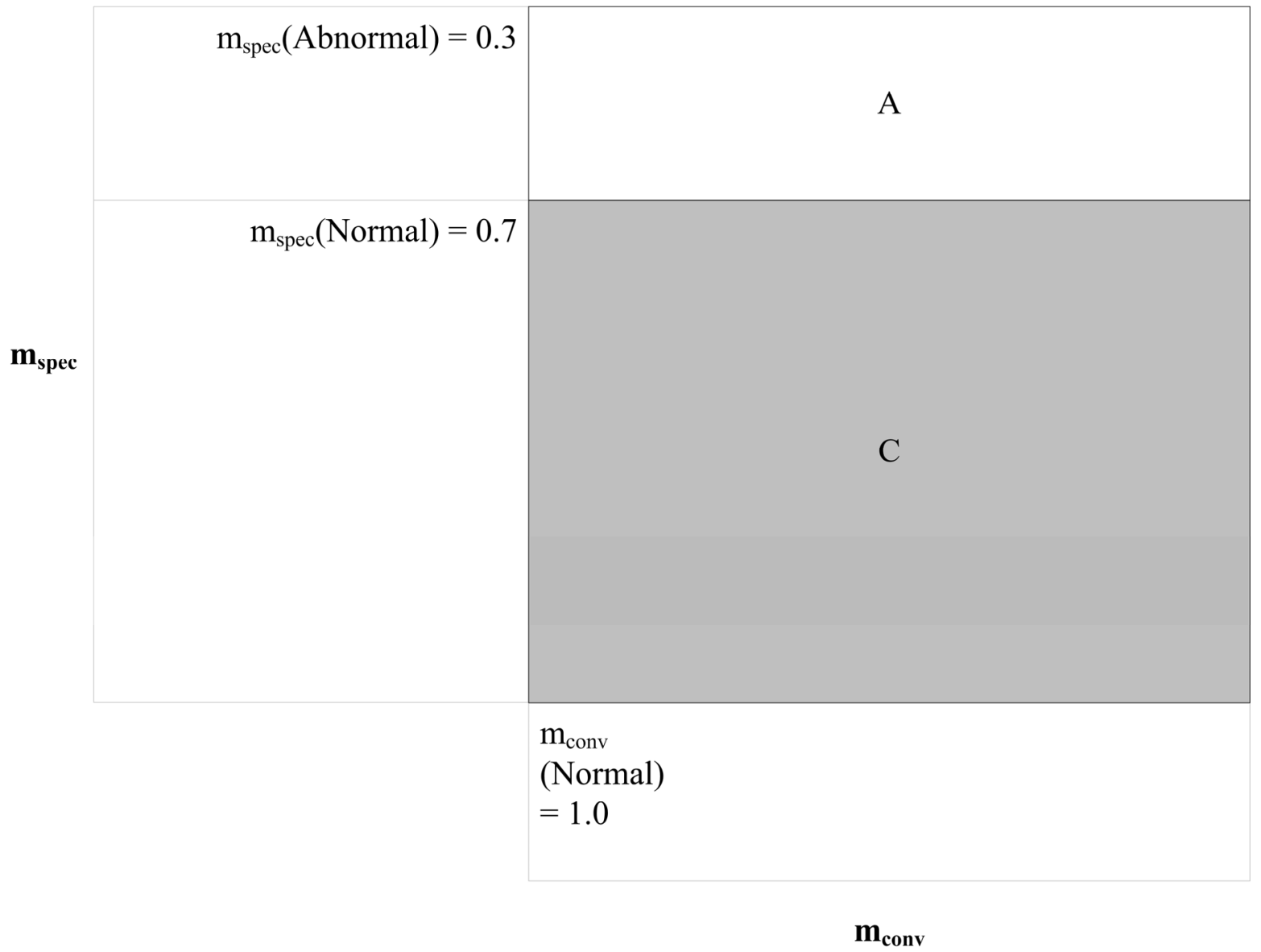

Figure 4. IDWMS-based example of a misleading Dempster's rule result

\subsubsection{Relation to Bayesian Probability Theory}

The Dempster-Shafer theory is a generalization of Bayesian probability theory, and Dempster's rule of combination is a generalization of Bayes' law (Shafer, 1976). The IDWMS's simplified implementation of Dempster's rule parallels Bayes' law:

$$
P(K \mid k)=\frac{P(k \mid K) P(K)}{P(k \mid K) P(K)+[1-P(k \mid K)][1-P(K)]}
$$

The prior probability, $P(K)$, and likelihood probability, $P(k \mid K)$, parallel the two intermediate mass assignment functions. The posterior probability $P(K \mid \mathrm{k})$ parallels the joint mass assignment function. However, the nature of the evidence is different between the two theories. In Bayes' law, a prior probability is updated with new evidence. In Dempster's rule, both pieces of evidence are obtained concurrently and combined. In addition, propositions can represent individual events or sets of events in the Dempster-Shafer theory (Shafer, 1976). This allows 
evidence to be assigned to sets of events without making any claims about the events within the sets. The Dempster-Shafer theory can thus represent uncertainty in a way not possible with traditional probability theory, where evidence can only be assigned to a single event (Sentz, 2002). However, this advantage of the Dempster-Shafer theory is not leveraged by the IDWMS.

The possibility for Dempster's rule to produce misleading results requires operators to supervise a detection system using the rule, and for operators to trust and rely upon its results appropriately. The following section discusses models of human-automation interaction that might inform the design of a display that supports the desired operator behaviour. 


\section{Human-Automation Interaction}

This section reviews the models with which human-automation interaction has been described in the literature, as well as the role of trust and reliance in this interaction. In addition, approaches to analyzing and modelling human-automation systems for display design are reviewed.

\subsection{Supervisory Control}

Supervisory control is a model of human-automation interaction in which a human operator supervises subordinate automation in the control of a system (Sheridan, 2006). Strictly speaking, supervisory control requires the automation to form a closed loop with the system ( $c$ and $d$ in Figure 5), on which the automation can independently act given operator-defined conditions. In a broader sense, the operator retains control of the system and the automation serves as a mediator by translating operator instructions into low-level control actions or by processing system information for operator consumption ( $b$ in Figure 5). In this latter interpretation, the automation and system form an open loop, as system feedback can only affect automation action through the operator. For both interpretations, there is a display portion and an action portion of the control loop, and the automation may work primarily on one portion or both. Display functions include processing system information for operator consumption or analyzing system information to suggest courses of action, while action functions include completing entire tasks or augmenting operator control actions.

The degree to which an automated system works on the display and action portions of the control loop was described by Sheridan and Verplank (1978) as a scale featuring 10 levels of automation. At the lowest level, the automation is not involved in the decision-making and control process, and the system is under fully manual control. At the highest level, the automation is completely autonomous in performing the entire process, from option generation to action selection and implementation. In the levels between, the operator's role in the decision-making process varies from selecting actions to approving or vetoing computer-generated solutions. 

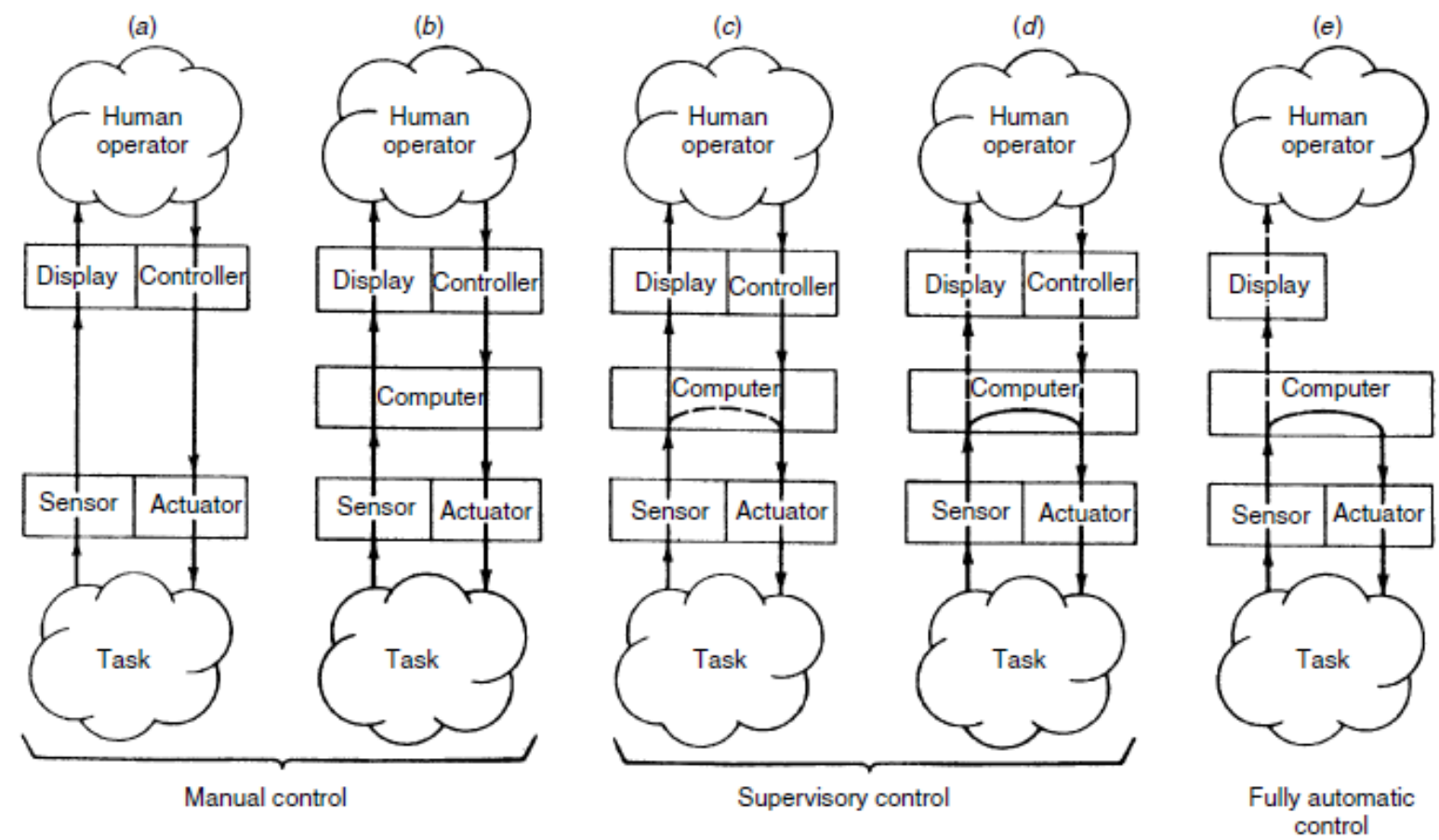

Figure 5. Definitions of supervisory control (from Sheridan, 2006)

Parasuraman, Sheridan, and Wickens (2000) extended these levels of automation by adding a dimension for automation types, which were adopted from the stages of a human information processing model (Wickens \& Hollands, 2000). Four types of information processing functions can be automated: information acquisition, information analysis, decision and action selection, and action implementation. A human-automation system can be characterized by assigning a level of automation to each of the four automation types (Figure 6). 
Information

Acquisition

Automation

Level
Information

Analysis

Automation

Level
Decision

Selection

Automation

Level
Action Implementation

Automation

Level

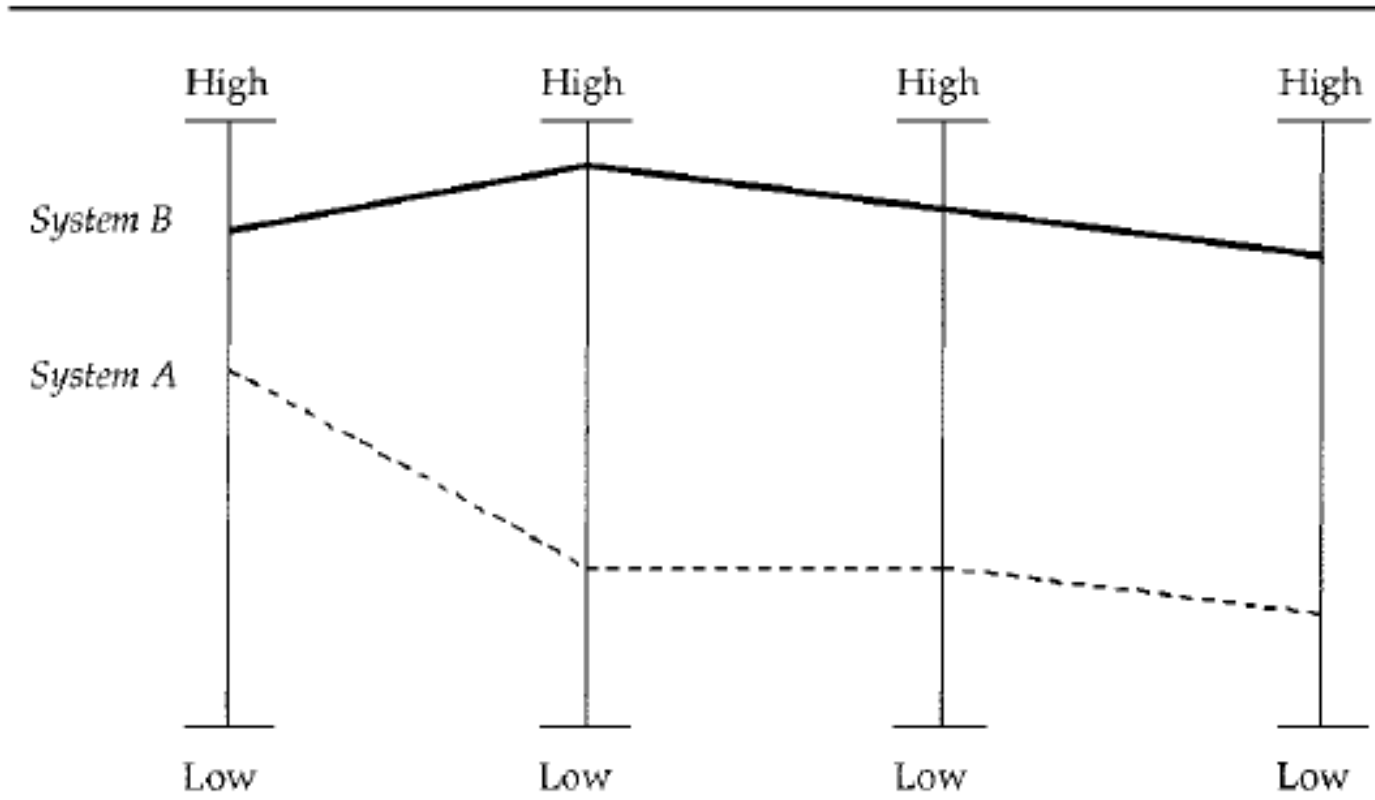

Figure 6. Types and levels of automation (from Parasuraman et al., 2000)

\subsection{Trust and Reliance}

It is impossible for operators to observe and reason about complex systems ${ }^{1}$ in a complete and certain fashion, which necessitates the use of automated aids. However, it is also impossible to have perfectly reliable automation that provides a complete representation of the system. This uncertainty requires operators to supervise and appropriately rely upon the automation in order to improve joint human-automation decision-making and system performance.

Under-reliance occurs when operators reject automation in situations where it is beneficial to engage it (Parasuraman \& Riley, 1997). Consequently, benefits of automation technical capability are not realized and the performance of the joint human-automation system suffers.

\footnotetext{
${ }^{1}$ Vicente (1999) provides a broad list of characteristics that increase the complexity of systems: Complex systems present a large problem space and are hazardous, highly coupled, automated, and subject to disturbances. Complex systems also have social dynamics: distributed workers with heterogeneous perspectives must work together to make the overall system successful. Interactions between workers and these systems are mediated by computers and surrounded by uncertainty.
} 
Joint performance also suffers with over-reliance, which occurs when operators engage automation in situations for which it was not designed (Parasuraman \& Riley, 1997). One possible consequence of over-reliance is automation bias, a decision-making bias that causes operators to focus excessively on an automated system's results and to fail to properly collect, acknowledge, or interpret information from other sources (Mosier \& Skitka, 1996).

Consequently, users may become complacent and fail to notice problems not detected by the aid, or follow automated information that is inappropriate or false. A user's trust in an automated system is one of several factors ${ }^{2}$ that mediate the degree to which he or she will rely upon the system: a higher degree of trust will cause the user to rely more heavily upon the automation (Parasuraman \& Riley, 1997). A means to supporting appropriate reliance is engendering appropriate trust in the automation (Lee \& See, 2004), and this is the approach taken here.

Lee and See (2004) assessed the appropriateness of trust along three dimensions: calibration, resolution, and specificity. Calibration refers to the degree of trust a person has in the automation relative to the automation's capabilities; over-trust occurs when a person's trust exceeds the system's capabilities. Resolution refers to how sensitive a person's trust is to differences in automation capability. Related to resolution is specificity, which refers to the amount of trust associated with a specific function of the automation. Specificity can also refer to changes in trust based on the time and situation when the automation is engaged. Good calibration, high resolution, and high specificity of trust support appropriate reliance on automation.

In order to appropriately trust an automated system, users must understand how capable the automation is at achieving their goals (Lee \& See, 2004). Automation capability can be described along two dimensions. The first varies in abstraction: purpose describes what the automation has been designed to do; process describes the automation's algorithms and operations used to achieve the automation's purpose; and performance refers to demonstrations of the automation's competency in achieving the operator's goals. Furthermore, inferences can be drawn between the three attributes of automation capability. For example, a user can estimate how well a system will perform given an understanding of its processes. If a user's inferences about automation capability are inconsistent with observed capability, then trust may suffer (Lee \& See, 2004). The

\footnotetext{
${ }^{2}$ In addition to trust, Parasuraman and Riley (1997) identified individuals' attitudes towards automation, mental workload, the cognitive overhead associated with engaging the automation, self-confidence, and risk as factors that influence automation usage.
} 
second dimension for describing automation capability varies in detail, ranging from individual components or modes to the entire system. Information at each level of abstraction and detail should be communicated to users to encourage good calibration, high resolution, and high specificity of trust. This information can be provided to users via displays, which is the object of design in this thesis.

\subsection{Implications for Decision Aids}

Given that the context of this thesis is the IDWMS, the current focus is on automated decision aids, which act primarily on the display portion of the supervisory control loop (Sheridan, 2006). Decision aids may acquire and integrate system information for the operator, and/or interpret information to estimate system state and suggest operator actions. In terms of Parasuraman et al.'s (2000) types and levels of automation, this thesis characterizes decision aids as having any level of information acquisition automation, high levels of information analysis automation, low to medium levels of decision selection automation, and no action implementation automation (Figure 7). At high levels of information analysis, decision aids may employ sophisticated but imperfect algorithms that cannot be easily understood by operators, necessitating the communication of automation capability so that operators can adapt their reliance upon the automation in a context-specific manner (Lee, 2006; Lee \& See, 2004). For example, the misleading results produced by Dempster's rule of combination in section 1.2 would require operator adaptation. 


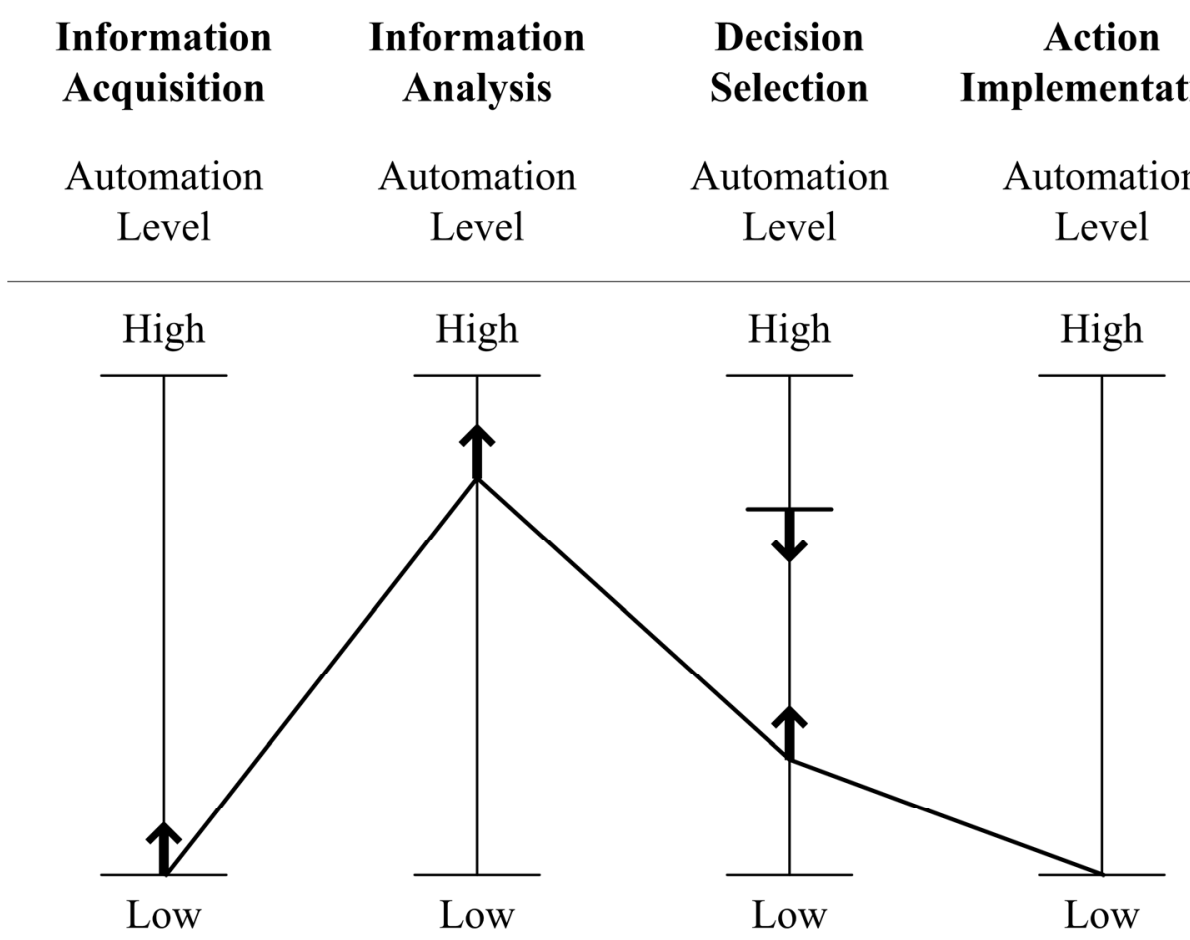

Figure 7. Characterization of automated decision aids using types and levels of automation (adapted from Parasuraman et al., 2000)

\subsection{Modelling Human-Automation Interaction}

Modelling human-automation systems can inform the display content required for supporting effective supervisory control. Two modelling approaches for human-automation systems are reviewed, but are found to have limitations for the current design goal of supporting appropriate trust in automation.

\subsubsection{Finite State Machines}

Degani and Heymann (2002) used finite state machines as a formal approach to describing human-automation interaction. Their approach requires three models to be constructed. First, a machine model is constructed that consists of the machine's legal and illegal states, as well as any possible transitions between the states. Next, a user model of the machine is constructed based on the user's mental model of the automation, as well as the transitions that the user is able to observe and trigger from the interface. Lastly, a composite model is constructed by combining the machine and user models. From the composite model, inconsistencies between the user model and machine model can be identified: the user model may contradict the machine model with respect to which states are valid, or the user model may prevent the user from triggering 
technically possible transitions and accessing legal states in the machine model. The objective of this analysis is to develop a user model and interface that provides users with the necessary information to determine the current and next state of the machine. In addition, the user model must not prevent the user from triggering possible transitions and accessing legal states.

The primary limitation of this approach is that it requires formal descriptions of the automation and user tasks, and creating such descriptions is not trivial (Heymann \& Degani, 2007). In addition, the approach's assumption that automation behaves deterministically may not always appear to hold from the operator's view of a complex work domain (Jamieson \& Vicente, 2005). With regards to the basis of trust in automation (Lee \& See, 2004), the transitions capture very detailed information about the rules that govern system behaviour. However, the approach appears to focus on communicating system states, and it is unclear how these transitions can be communicated to the user to foster appropriate trust.

\subsubsection{Multilevel Flow Models}

Lind (1999) proposed multilevel flow modelling to represent automated systems using three connected levels: the component level, the function level, and the intentional level. The component level of the model describes the physical components of the system, and the interactions between these components are described at the functional level. The intentional level describes the goals and objectives of the system. These three conceptually different views of the same system are connected using a set of eight relations that includes goals, means, and aggregation. Multilevel flow modelling also represents functions by modelling their mass and energy flows using a set of system functions, such as sources, sinks, and balances.

The relations between components, functions, and objectives facilitate planning and fault diagnosis actions (Lind, 1999). From these relations, it can be reasoned why a particular function is performed, what the manner and the means to achieving the function are, and under what circumstances the function should be performed. By connecting the plant designer's intentions, the plant's processes, and the plant's components in a single model, the multilevel flow modelling approach limits the complexity of planning and diagnostic reasoning, and supports the gathering of information requirements. However, creating a multilevel flow model is complex (Lind, 1999) and there is not enough published material to guide its application or to judge its applicability for designing displays that support appropriate trust and reliance on automation. 
The discussed limitations of these analyses hinder their application to the current design goal of designing for appropriate reliance upon a decision aid. In addition, neither approach informs the design of graphic forms that present the identified information in a coherent manner that corresponds to the work domain. Such guidance is required to ensure that operators can extract the information relevant to achieving a goal or task (Bennett, Nagy, \& Flach, 2006). In the next section, Ecological Interface Design is proposed as an approach to designing displays that support effective supervision of decision aids. 


\section{Ecological Interface Design}

Ecological Interface Design (EID) is an approach to interface design that aims to identify the constraints within complex work domains and make them directly perceptible to operators (Burns \& Hajdukiewicz, 2004; Vicente \& Rasmussen, 1990; Vicente \& Rasmussen, 1992). Constraints are relationships that must hold true for a system to be operating as intended. EID emphasizes the study of environmental constraints over operators' cognitive constraints because the former apply to all operators, while the latter can vary by individual. Examples of ecological constraints include the laws of thermodynamics. Given that these constraints must hold true, they can be used to detect and diagnose both routine and unanticipated system faults. This section introduces EID's analytic framework, the abstraction-decomposition space, which informs display content. EID's use of the skills, rules, knowledge (SRK) framework (Rasmussen, 1983) to guide display form is also introduced. This thesis proposes that EID can be used to design a decision aid display that supports appropriate trust by presenting information about the aid's capability in a directly perceptible manner.

\subsection{Work Domain Analysis}

The EID approach begins with a work domain analysis that models a system using a twodimensional space called the abstraction-decomposition space (ADS; Burns \& Hajdukiewicz, 2004; Vicente, 1999). A work domain is defined by Vicente (1999) to be "the system being controlled, independent of any particular Worker, Automation, Event, Task, Goal, or Interface” (p. 10). From this model, analysts can extract the information required to monitor the constraints that govern system behaviour.

\subsubsection{Decomposition Hierarchy}

The decomposition hierarchy is the horizontal dimension of the ADS and decomposes the work domain according to the part-whole relationship (Figure 8; Burns \& Hajdukiewicz, 2004; Vicente, 1999). At the lowest level of decomposition, the system is modelled as a collection of individual components. At higher levels, these components are aggregated into meaningful subsystems, which are further aggregated into the overall system. The labels for the subsystem and component levels are generic because the part-whole relationship affords some flexibility in dividing a system. A decision aid might be decomposed into subsystems responsible for different modes of operation, while another approach might isolate subsystems responsible for different 
stages of information processing. A decomposition approach might be chosen by studying how the system was designed, or by determining if specific purposes can be attributed to particular subsystems.

\begin{tabular}{|l|l|l|l|}
\hline & System & Subsystems & Components \\
\hline Functional Purposes & & & \\
\hline Abstract Functions & & & \\
\hline Generalized Functions & & & \\
\hline Physical Functions & & & \\
\hline Physical Form & & & \\
\hline
\end{tabular}

Figure 8. An empty abstraction-decomposition space (ADS; Vicente, 1999)

\subsubsection{Abstraction Hierarchy}

The abstraction hierarchy $(\mathrm{AH})$ is the vertical dimension of the ADS, and is a hierarchy built upon the structural means-end relationship (Figure 8; Vicente, 1999). This relationship requires that the parent node be achieved by its children nodes. Furthermore, each node, as well as the relationship itself, must be a relatively permanent property of the work domain. In the $\mathrm{AH}$, each node is a constraint that governs meaningful action in the work domain. These constraints are organized into the AH's five levels of abstraction. The constraints vary conceptually between the levels, but each level provides a complete description of the work domain. The constraints in adjacent levels of abstraction are connected using the described structural means-end relationship. From the highest to lowest levels of abstraction, the AH is comprised of the work domain's functional purposes, abstract functions, generalized functions, physical functions, and physical form.

Functional purposes are the overall intentions that the work domain was designed to achieve (Vicente, 1999). For comparison, goals are desired states of the work domain that operators wish 
to achieve and are thus dynamic properties of operators. Since the AH is independent of agency, goals are not represented in the hierarchy.

Functions are structural properties of the work domain that are relevant to both achieving the domain's functional purposes and achieving the operators' goals (Vicente, 1999; Burns \& Hajdukiewicz, 2004). Abstract functions are the work domain's underlying flows and causal relationships. Although a power plant (Burns, 2000), pasteurizer (Reising, 2002), and car (Burns \& Hajdukiewicz, 2004) have different functional purposes, they can all be described fundamentally as flows of mass and energy governed by the laws of thermodynamics. As an information processing system, a decision aid can be described fundamentally as flows of information that may be governed by mathematical theories. For example, a decision aid that uses statistical inference might be governed by Bayes' law, which dictates the relationship between prior and posterior probabilities.

Generalized functions are the work domain's processes for transforming mass, energy, or information to achieve the work domain's abstract functions. Typically, this level contains system processes such as fission, condensation, and combustion (Burns \& Hajdukiewicz, 2004). For a decision aid, generalized functions can be considered its implementation-specific algorithms. Physical functions are the physical components of the work domain and their capabilities for achieving the work domain's generalized functions. The physical form of each component is described in terms of its appearance and location. For a decision aid, these physical levels of abstraction may include available sensors, their capabilities, and their placement.

The levels of abstraction also vary in detail, as higher levels of abstraction describe the work domain with less resolution than lower levels of abstraction (Vicente, 1999). Thus, the abstraction and decomposition hierarchies are not entirely independent: higher-level goals are usually associated with the overall system, while specific processes are usually studied in terms of the smaller subsystems or individual components (Burns \& Hajdukiewicz, 2004). By connecting the purposes and functions in adjacent levels of abstraction using the structural means-end relationship, the $\mathrm{AH}$ is a purpose-oriented representation of the work domain (Vicente, 1999). This characteristic helps operators survey the work domain at a higher level of abstraction to identify a purpose or function that is of current interest, and then selectively examine the functions used for its achievement. 


\subsubsection{Modelling Automation}

Although the previous section showed how the levels of abstraction might represent a decision aid's purposes and functions, there are arguments against using the ADS to model automation. First, Vicente (1999) defines a work domain as being "the system being controlled, independent of any particular Worker, Automation, Event, Task, Goal, or Interface" (p. 10). However, the excluded items may be more broadly interpreted as concepts related to control agency. With this interpretation, decision aids do not meet the exclusion criteria, as they have no control agency according to Lee's (2006) characterization of automated agents, and according to the characterization of decision aids used in this thesis (based on Parasuraman et al.'s (2000) taxonomy; section 2.3).

Lind (2003) argues that automated control systems must be considered in models of a work domain, but that the $\mathrm{AH}$ is an insufficient framework for doing so. An unsuccessful $\mathrm{AH}$ representation of biological control systems (Miller \& Sanderson, 2000) is cited as an example. Lind (2003) suggests that two hierarchies are required to properly describe the role of control systems: one to represent the physical processes and another to represent the control processes. However, Lind (2003) finds that the means-end relation is insufficient for joining these two hierarchies and describing how the automation controls the physical processes. Mazaeva and Bisantz (2007) used two ADSs to represent a camera and its automated control systems, which is similar to the dual AH approach suggested by Lind (2003). The authors linked functions from the two ADSs using a relationship that identified the camera functions controlled by a given control system function.

Conversely, others have suggested that automation be included in the AH if it is within the reasoning spaces of operators. To study the inclusion of sensor automation in the $\mathrm{AH}$, Burns, Bisantz, and Roth (2004) compared two models of a similar work domain performed by separate groups of analysts. One group modelled sensors as components in the AH, while the other did not. In the case of sensor inclusion, sensor management was to be supported by the interface, thereby placing sensors within the system to be controlled and within Vicente's (1999) definition of a work domain. Sensors were excluded by the other analysts to encourage operators to consider more than the sensed information when reasoning about the work domain. In summary, both groups' decisions were made to encourage certain reasoning spaces from workers. Burns et 
al. (2004) recommended that in cases where automation must be monitored, configured, or controlled, it should be represented in the $\mathrm{AH}$.

Similarly, Naikar, Hopcroft, and Moylan (2005) recommended that the work domain be modelled such that it is consistent with the reasoning space of the workers. If automation is within operators' reasoning spaces, then automation should be included in the model. However, this raises the possibility that the resulting model adopts a descriptive and task-based perspective. This is problematic because operators do not always have an accurate or complete understanding of a complex work domain's functions and purposes. An excessive emphasis on supporting workers' current reasoning spaces may detract from the benefits of conducting an ecological constraint-based analysis.

\subsubsection{Representing Automation Capability}

Lee and See (2004) suggested that displays for automated systems may be used to support appropriate trust. For displays to achieve this goal, they must display the necessary information about automation capability in an appropriate format. The authors noted that the informational basis of trust in automation and the $\mathrm{AH}$ share common dimensions of attributional abstraction

and system detail. The authors further suggest that the AH could be used to analyze an automated system's capabilities and identify the necessary information requirements for supporting appropriate trust. This section considers how the AH might be used to do so.

Information about automation purpose describes why the automation was developed and the designer's intentions for the use of the automation (Lee \& See, 2004). For appropriate trust to occur, purpose information must be provided to help operators understand the intended use of the automation. Both automation purposes and functional purposes describe the intentions of a system as being relatively fixed properties that are designer-dependent and operator-independent. For this reason, functional purposes might be best suited to representing automation purposes.

Information about automation process describes how the automation operates (Lee \& See, 2004). To trust an automated system appropriately, operators must understand its operations and algorithms, as well as how capable they are in achieving the operators' goals in a particular situation. The AH might help operators understand an automated system's process by providing three conceptually different representations of its functions. The abstract, generalized, and physical functions are proposed to be a decision aid's underlying theories, algorithms, and 
physical components. Furthermore, the purpose-oriented nature of the AH that supports problemsolving (Vicente, 1999) might also support understanding of automation process. Operators can first identify a functional purpose (i.e., intended use) of interest before selectively examining the functions used for its achievement. The means-end relations might also be used in the opposite direction to help operators understand how capable the automation is in achieving the operators' goals in a particular situation. For example, if a sensor is deemed to be unreliable, then its effects on higher level functions and purposes can be identified.

In the same ways, the purpose-oriented nature of the AH might also improve the inferences that operators may make about an automated system's purposes based on their understanding of its processes (and vice versa). The system's performance might also be inferred given an understanding of its process. If these inferences do not match observed capability, then trust will suffer (Lee \& See, 2004). For example, a user may expect a signal detection (a functional purpose) system to employ certain mathematical theories (abstract functions), algorithms (generalized functions), or sensors (physical functions).

Automation performance is the automation's demonstrations of competence (Lee \& See, 2004). Such demonstrations include its current and past operation, as well its reliability and predictability. Descriptions of an automated systems performance might be extracted from the $\mathrm{AH}$ as information requirements, which measure the state and achievement of the work domain's functions (Burns \& Hajdukiewicz, 2004). Some analysts have suggested that measures of functional purpose achievement should be included at the abstract function level (in which case, this level of abstraction is renamed "Values and Priority Measures"; Naikar et al., 2005; Reising, 2000). However, this approach conflicts with the means-end relationship between levels of abstraction. For example, high reliability would not be a means to achieving signal detection.

Duez et al. (2006) created an AH for an automatic database memory manager to gather information requirements that described automation capability. The analysts' objective was to engender appropriate operator trust in the automation's actions and performance. The functional purpose level of the $\mathrm{AH}$ was found to provide purpose-related information requirements, while the remaining rows of the $\mathrm{AH}$ provided process-related information requirements. Variables measuring the state of the identified purposes and functions were used to describe automation performance. Mazaeva and Bisantz's (2007) automation AH described how the automation's 
subsystems and components achieved its purposes, acknowledging the presence of purpose- and process-related information.

\subsection{Ecological Graphic Forms}

In addition to identifying display content, display design requires that the content is displayed in appropriate forms that are appropriately structured. In EID, a display's graphic forms and structure should support each level of cognitive control in the SRK framework (Rasmussen, 1983; Vicente \& Rasmussen, 1992). To support skill-based behaviour, operators should be able to act on the system directly from the display, and the displayed information and control movements should be consistent with each other. To support rule-based behaviour, the display's graphic forms should be perceived as consistent one-to-one mappings to the work domain's constraints. To support knowledge-based behaviour, the display content and forms should be organized according to the $\mathrm{AH}$, as it is psychologically relevant representation of the work domain during problem-solving. Due to the cognitively demanding nature of knowledge-based behaviour, EID strives to limit its use to handling unanticipated situations when workers' rules may not apply (Vicente \& Rasmussen, 1992). To design visualizations that make system constraints directly perceptible and support rule-based behaviour, research on the representationaiding approach to display design can be referenced.

Representation-aiding considers two design problems (Bennett et al., 2006). First, the display's content must correspond to the work domain or problem space. EID uses the AH to identify a work domain's purposes and functions, as well as the means-end relationships between them, to provide operators with the necessary information to reason about the domain. Second, the display must present this information in a coherent fashion to the operator. The design goal is for visual representations to be perceived as accurate mappings to the work domain's relationships. Object displays may be used to achieve such mappings (Bennett \& Flach, 1992; Pomerantz \& Pristach, 1989; Vicente \& Rasmussen, 1990; Wickens \& Carswell, 1995). Object displays integrate several low-level codes ${ }^{3}$ into a single object, which may also give rise to emergent features. An emergent feature is a higher-level visual feature that is not a property of any individual low-level code, but a readily perceived feature that becomes apparent when several low-level codes are integrated. Emergent features can be used to represent the identified work

\footnotetext{
${ }^{3}$ A code is the visual dimension (such as the length or angle of a line) used to represent the value of a variable.
} 
domain constraints. Effective representation-aiding allows operators to directly perceive the state of a work domain function or purpose (Bennett, Nagy, \& Flach, 2006).

For example, if the height and width of a rectangle represent mass and acceleration, then the emergent feature of area maps to force (Figure 9). More complex and practical applications of emergent features were demonstrated by Barnett and Wickens (1988), who used rectangles to represent the information worth of multiple diagnostic cues to be used by participants in a decision-making task. Wickens and Andre (1990) used a rectangle formed by several lines to represent the overall state of a system based on the states of individual components. Vicente and Rasmussen (1990) mapped algebraic equations of mass and energy balances to geometric representations of a trapezoid and triangle. The effectiveness of such displays depends on the quality of the mapping between the emergent feature and underlying work domain relationship, and not simply on the presence of an object (Bennett \& Flach, 1992; Pomerantz \& Pristach, 1989; Wickens \& Carswell, 1995). Furthermore, according to the Proximity Compatibility Principle (Wickens \& Carswell, 1995), integrated displays may only be beneficial if operators are concerned with the visualized integration task. Emergent features may prevent operators from focusing their attention on the lower level codes. However, this disadvantage does not always occur (Barnett \& Wickens, 1988; Bennett \& Flach, 1992).

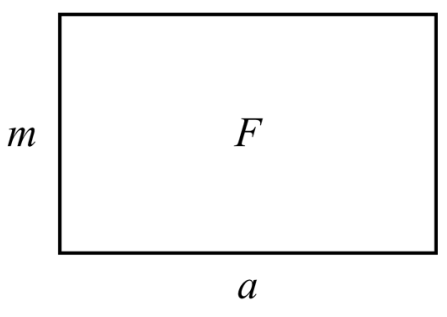

Figure 9. Mapping $F=m a$ to an emergent feature

This thesis focuses upon using such integrated displays to give operators direct representations of a decision aid's functions and purposes because the literature has suggested such displays to be useful for supporting understanding of, and appropriate trust in, automation. Lee (2006) suggests that automation-related information can be integrated with traditional information requirements to support appropriate trust without overwhelming operators with information. To help operators understand complex process control algorithms, Guerlain, Jamieson, Bullemer, and Blair (2002) suggested the use of emergent features to represent higher-order relationships. 


\section{Work Domain Analysis for the Intelligent Drinking Water Monitoring System}

This section describes the investigation and modelling of the IDWMS. The resulting ADS is presented along with the extracted information requirements to be displayed.

\subsection{Knowledge Acquisition}

To gain an overview of the decision aid, unstructured interviews were held with IDWMS designers and developers (Hoffman, Shadbolt, Burton, \& Klein, 1995). In the initial interviews, these experts tended to describe the IDWMS primarily in terms of its intended uses and physical components. Some of the aid's analyses were also revealed, but only at a very general level of detail. The unstructured nature of these discussions, as well as their separation into several sessions, made it difficult to combine the identified concepts into a unified understanding of the IDWMS. Furthermore, the analyst was unable to effectively ask probing questions given his unfamiliarity with the domain. At the same time, the experts sometimes incorrectly assumed that the analyst had an understanding of domain concepts (Hoffman et al., 1995), or were reluctant to delve into technical details of the IDWMS.

Structured interviews (Hoffman et al., 1995) provided a more unified understanding of the initially identified concepts. In these sessions, experts walked through the processes for acquiring and analyzing data. Documentation analysis helped generate probing questions for these sessions (Hoffman et al., 1995). Technical reports identified the specific operations and algorithms employed by the IDWMS and similar technologies. Lecture materials and textbooks provided a fundamental understanding of these algorithms. In particular, identifying and understanding the Dempster-Shafer theory helped structure interviews, as the IDWMS functions could be discussed in terms of this underlying theory. Finally, focused discussions with the experts (Cooke, 1994) around display prototypes helped verify the analyst's understanding of the system.

\subsection{Decomposition Hierarchy}

The IDWMS decomposition is presented in Figure 10. The system was first decomposed into sensor site subsystems. The IDWMS has sensor sites installed throughout the distribution network. Each site acquires conventional data (total chlorine, $\mathrm{pH}$, conductivity, oxidation reduction potential, and turbidity), as well as a spectrum. Thus, a sensor site subsystem 
represents the components and functions responsible for acquiring and analyzing the data for a particular sensor site. Consequently, each sensor site subsystem was decomposed into its conventional and spectroscopic analyses. For the final level of decomposition, the conventional and spectroscopic analyses were decomposed into their physical components. Note that there is a common processing unit and database for all subsystems.

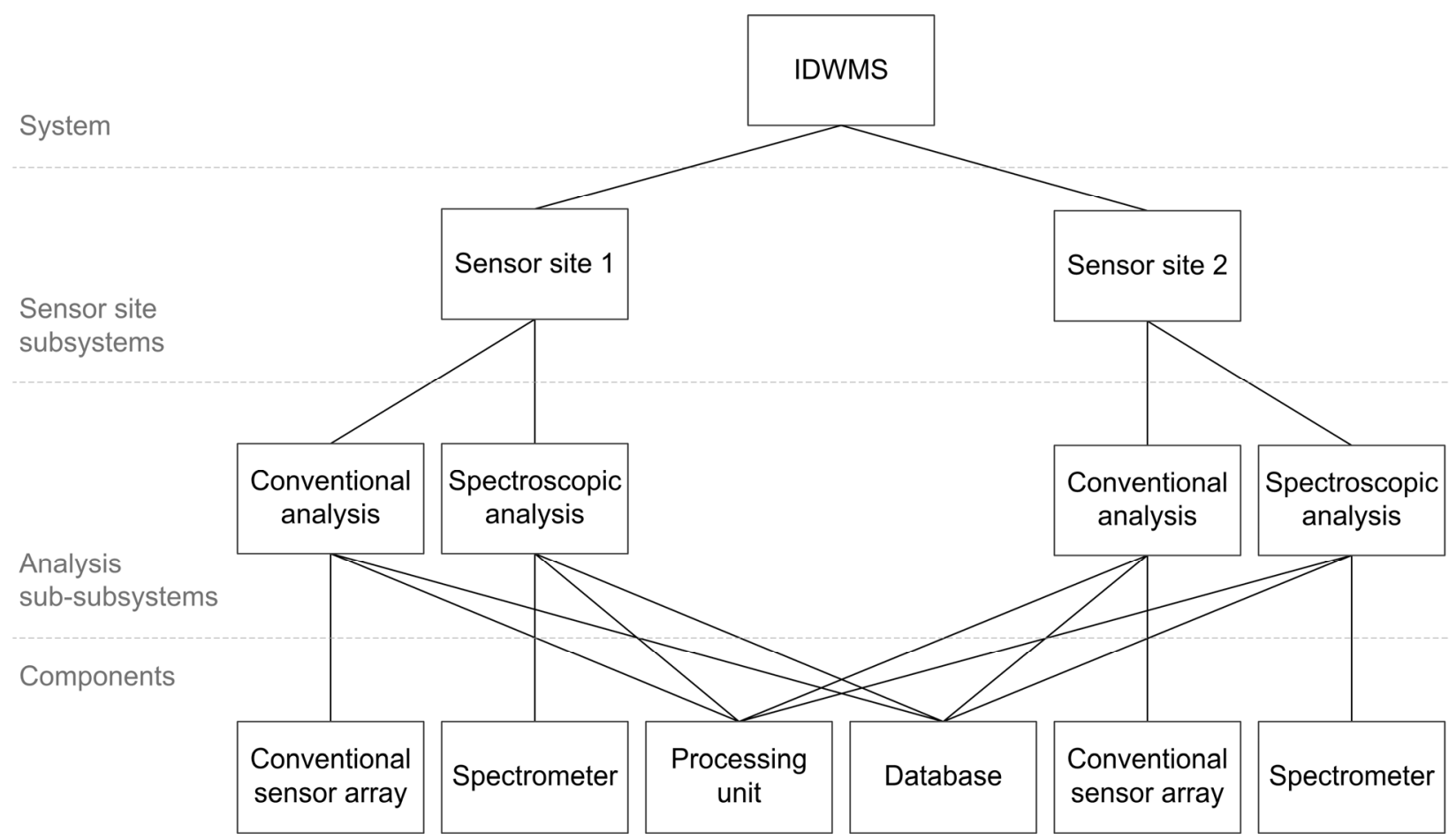

Figure 10. IDWMS decomposition hierarchy

\subsection{Abstraction-Decomposition Space}

Using the described decomposition hierarchy, the AH levels of abstraction were populated to create the ADS shown in Figure 11 and Figure 12.

\subsubsection{Functional Purposes}

The functional purpose of the entire IDWMS system is to detect water anomalies in a distribution network. However, algorithms have not yet been developed for the IDWMS to infer the overall water quality of a distribution network by integrating the detection results from each sensor site. Thus, the purpose of the overall system is to detect anomalies at any of its sensor sites. This purpose is repeated at a per-site resolution at the sensor site level of decomposition. Additional detail about this functional purpose is found at the analysis level of decomposition, where the functional purposes of the conventional and spectroscopic detection analyses are 
represented. The conventional analysis is designed to detect anomalies by comparing conventional water quality parameter readings to threshold values configured by control room operators. The second analysis uses spectroscopy to determine if sampled water differs from a normal baseline and to identify any contaminants that may exist in the sample. Identifying the purposes that the IDWMS has been designed to achieve may help operators understand its intended use and how well it is able to achieve their goals. For example, while operators are likely concerned with assessing water quality for the entire distribution network, the IDWMS can only assess water quality at locations with a sensor site.

\subsubsection{Abstract Functions}

The abstract functions for a decision aid were suggested to be its underlying information flows and mathematical theories. The IDWMS is designed to use the Dempster-Shafer theory to fuse sensor data. Thus, information must be in the form of mass assignments, and information must be combined according to Dempster's rule of combination. At the analysis level of decomposition, two Dempster-Shafer theory entries exist to emphasize the presence of two different types of information and analyses. Separating the entries also communicates the associative and commutative properties of Dempster's rule (allowing multiple mass assignment functions to be combined in any sequence; Shafer, 1976). By describing the Dempster-Shafer theory, this level of abstraction describes the IDWMS algorithms in a more fundamental manner. Providing operators with conceptually different descriptions of the IDWMS's process may support a better understanding of its capability. For example, the potential for Dempster's rule to produce misleading results is associated with this level of abstraction. 


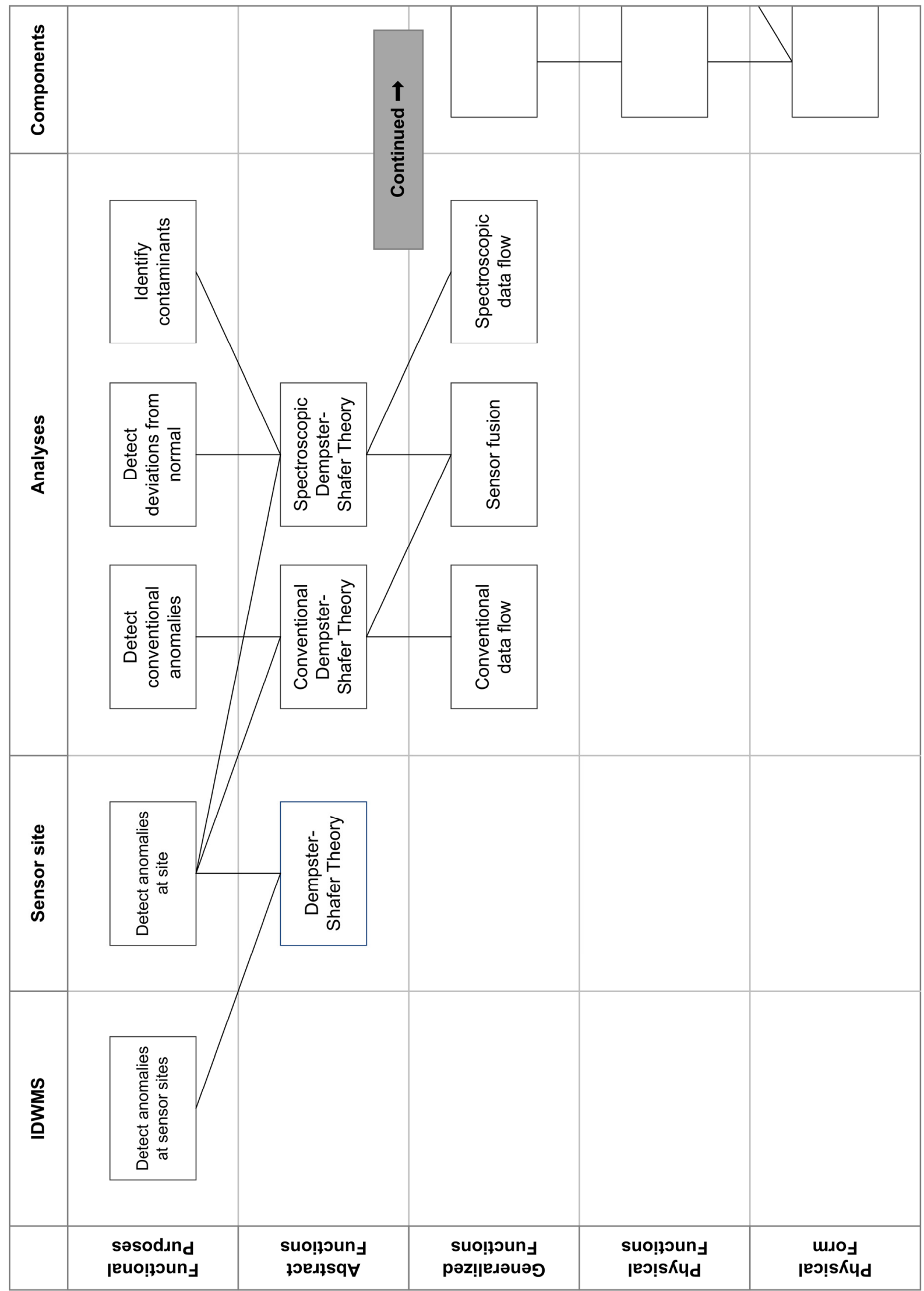

Figure 11. IDWMS abstraction-decomposition space 


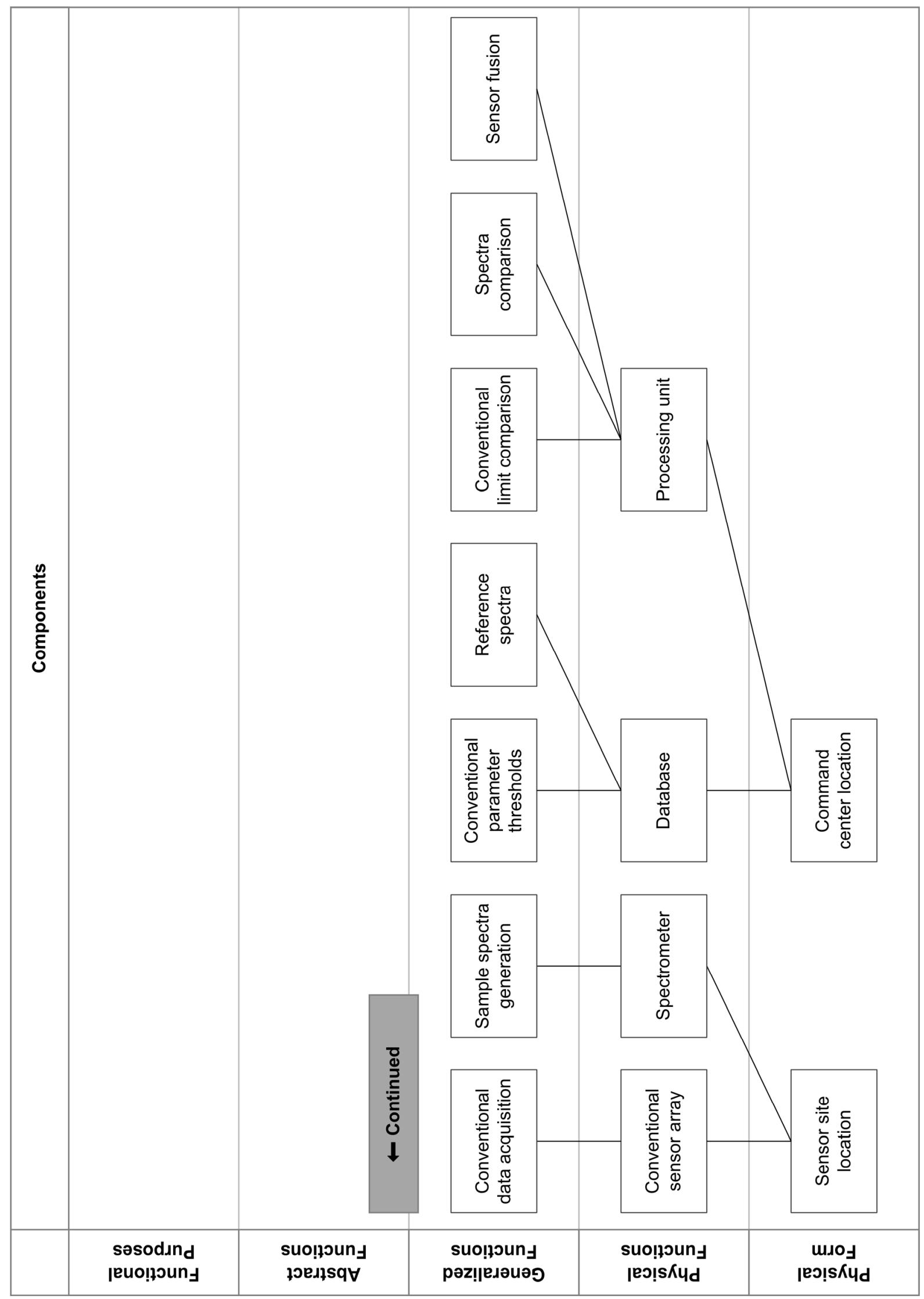

Figure 12. IDWMS abstraction-decomposition space (continued) 


\subsubsection{Generalized Functions}

The IDWMS's generalized functions at the analysis level of decomposition are the spectroscopic and conventional information flows that provide the mass assignments needed to achieve the Dempster-Shafer theory. An algorithm to achieve Dempster's rule of combination is also required. At the component level, the spectroscopic and conventional information flows are modelled with greater resolution. Each flow contains an information source (the acquisition of parameter values and spectra), an information store (conventional parameter thresholds and reference spectra), and a process that compares acquired and stored information to produce mass assignments. The algorithm entry is repeated at the component level of decomposition because each cell must be a complete representation of the system, and the algorithm entry could not be further decomposed in a conceptually consistent or meaningful manner. In comparison with the abstract function level, this level provides a more implementation-specific description of the algorithms and reveals additional process information about IDWMS capability. For example, a contaminant can only be identified if its spectrum has been stored in the IDWMS database.

\subsubsection{Physical Functions and Physical Forms}

Finally, the physical function represents the physical components of the IDWMS. Each sensor site consists of a conventional sensor array and spectrometer. The acquired data is transmitted to a central processing unit and database for analysis. The locations of each component are shown at the physical form level. The appearance of sensor arrays can be further described using a picture of the site, but none is included in the current ADS. Representing the physical functions and form is important because they are the available means to achieving the higher level functions and purposes. The IDWMS can only measure parameters for which it has sensors, limiting the information available for inferring the presence of water anomalies. The location of sensor sites in a distribution network is especially important because their cost prohibits complete coverage of the network. For locations without sensors, uncertainty about water quality increases given the complexity of distribution networks. The IDWMS lacks the algorithms to integrate information from multiple sensor sites, leaving operators to infer water quality based on the results of nearby sensor sites.

\subsection{Information Requirements}

To apply the ADS in design, each entry in the model is studied to determine the information requirements needed to measure the entry, as well as any relationships between information 
requirements. Since each level of abstraction describes the work domain using a different set of concepts, the measures extracted from each level will also differ conceptually (Burns \& Hadjukiewicz, 2004). Variables from the functional purpose level measure how effectively the system has achieved its designed purposes. Abstract functions are measured in terms of measuring flows, such as the amount of mass, energy, or information entering and leaving the system. In Dempster's rule, the normalization factor can be considered lost information. Generalized function variables measure the processing and transformations of mass, energy, or information in the system. For example, raw sensor readings undergo noise reduction and are compared with stored values to generate mass assignments. Physical function variables measure the operational state of the physical components, while physical form variables measure any relevant physical attributes of the component. These measures reveal how effectively the system's processes and purposes are being achieved. For example, if a sample spectrum is only compared to two contaminants' reference spectra, then the IDWMS has performed poorly in identifying contaminants. Such demonstrations of competence correspond to Lee and See's (2004) performance attribute of automation capability for supporting appropriate trust.

Table 1 presents the information requirements extracted from the IDWMS ADS. The availability of each information requirement is indicated in the rightmost column, as some variables are unavailable because they are not stored by the IDWMS. In cases where information requirements are unavailable, operators may be unable to determine the state of a function or purpose. Consequently, they may also be unable to assess the true capability of the automation in a particular situation. 
Table 1. IDWMS information requirements

\section{ID Information Requirement}

Available?

Functional Purposes

\begin{tabular}{|c|c|c|}
\hline 1 & Final normal/abnormal conclusion & Yes \\
\hline 2 & Reliability of final anomaly conclusion & No \\
\hline 3 & Conventional normal/abnormal conclusion & Yes \\
\hline 4 & Reliability of conventional anomaly conclusion & No \\
\hline 5 & Spectroscopic normal/abnormal conclusion & Yes \\
\hline 6 & Reliability of spectroscopic anomaly conclusion & No \\
\hline 7 & Reliability of spectroscopic contaminant identification & No \\
\hline 8 & Contaminant and concentration with highest belief of existence & Yes \\
\hline \multicolumn{3}{|c|}{ Abstract Functions } \\
\hline 9 & Final joint mass assignment that sampled water is normal & Yes \\
\hline 10 & Final joint mass assignment that sampled water is abnormal & Yes \\
\hline 11 & Final normalization factor & Yes \\
\hline 12 & Conventional aggregate joint mass assignment that sampled water is normal & Yes \\
\hline 13 & Conventional aggregate joint mass assignment that sampled water is abnormal & Yes \\
\hline 14 & Conventional normalization factor & Yes \\
\hline 15 & Mass assignment functions for each conventional sensor & Yes \\
\hline 16 & Spectroscopic joint mass assignment that sampled water is normal & Yes \\
\hline 17 & Spectroscopic joint mass assignment that sampled water is abnormal & Yes \\
\hline 18 & Mass assignment for the existence of each contaminant & Yes \\
\hline 19 & Spectroscopic normalization factor for each comparison & No \\
\hline 20 & Mass assignment functions for each spectrometric comparison & No \\
\hline 21 & Dempster-Shafer theory propositions & Yes \\
\hline \multicolumn{3}{|c|}{ Generalized Functions } \\
\hline 22 & Conventional parameters measured & Yes \\
\hline 23 & Noise-reduced readings for each conventional parameter & Yes \\
\hline 24 & Raw readings for each conventional parameter & No \\
\hline 25 & Stored upper and lower limits for each conventional parameter & Yes \\
\hline 26 & For each conventional parameter, the difference between its sensor reading and its & Yes \\
\hline
\end{tabular}




\begin{tabular}{|c|c|c|}
\hline & upper and lower limits & \\
\hline 27 & Raw sample spectra & No \\
\hline 28 & Sample absorption spectrum & Yes \\
\hline 29 & List of contaminants with stored reference spectra & Yes \\
\hline 30 & Contaminants (and concentration) that have been compared to the sample & Yes \\
\hline 31 & Processed contaminant reference spectra compared to sample spectrum & No \\
\hline 32 & $\begin{array}{l}\text { Amount of difference between contaminant reference spectra and sample } \\
\text { spectrum }\end{array}$ & No \\
\hline 33 & Amount of difference between baseline spectrum and sample spectrum & No \\
\hline 34 & For each spectra comparison, absorption wavelengths compared & No \\
\hline 35 & Baseline absorption spectrum & Yes \\
\hline \multicolumn{3}{|c|}{ Physical Functions } \\
\hline 36 & Spectrometer status & Yes \\
\hline 37 & Spectrometer light source status (on/off) & Yes \\
\hline 38 & Spectrometer limitations (measured wavelengths) & Yes \\
\hline 39 & Conventional sensor limitations (measured range of values) & No \\
\hline 40 & Status of each sensor in array & Yes \\
\hline 41 & Status of processing unit & No \\
\hline 42 & Status of database & No \\
\hline 43 & Status of connection between each sensor site and the processing unit & No \\
\hline \multicolumn{3}{|c|}{ Physical Forms } \\
\hline 44 & $\begin{array}{l}\text { Location of each sensor site with respect to the distribution network (e.g., pumps, } \\
\text { filtration plants, storage) }\end{array}$ & Yes \\
\hline 45 & Location of processing unit & Yes \\
\hline 46 & Location of database & Yes \\
\hline
\end{tabular}


In addition to individual information requirements, the ADS entries and means-end links can be examined to extract relationships that exist between variables. At a basic level, many variables are constrained by threshold values. At a higher level, several variables may be related according to concepts such as the laws of thermodynamics or Dempster's rule of combination. The relationships identified for the IDWMS are presented in Table 2. Unavailable or partially available relationships are the IDWMS algorithms and operations that could not be studied in detail given the limited access to system designers and developers.

Table 2. IDWMS constraints

\begin{tabular}{|c|c|c|}
\hline ID & Information Requirement & Available? \\
\hline \multicolumn{3}{|c|}{ Single Variable Constraints } \\
\hline 47 & For every mass assignment, $0 \leq$ mass assignment $\leq 1$ & Yes \\
\hline 48 & $0 \mathrm{mg} / \mathrm{L} \leq$ concentration of any given contaminant & Yes \\
\hline 49 & $0 \mathrm{NTU} \leq$ turbidity & Yes \\
\hline 50 & $0 \mathrm{mg} / \mathrm{L} \leq$ free chlorine & Yes \\
\hline 51 & $0 \mu \mathrm{S} / \mathrm{cm} \leq$ conductivity & Yes \\
\hline 52 & $0 \leq \mathrm{pH} \leq 14$ & Yes \\
\hline \multicolumn{3}{|c|}{ Multivariate Constraints } \\
\hline 53 & $\begin{array}{l}\text { If } \mathrm{m}(\text { water is abnormal) }>\mathrm{m} \text { (water is normal), then the conclusion is that the } \\
\text { water is abnormal (and vice versa) }\end{array}$ & Yes \\
\hline 54 & Sum of all mass assignments must equal 1 for each information source & Yes \\
\hline 55 & Dempster's rule of combination & Yes \\
\hline 56 & $\begin{array}{l}\text { For each conventional parameter, the relationship between its reading and its } \\
\text { normal/abnormal mass assignments }\end{array}$ & Partial \\
\hline 57 & $\begin{array}{l}\text { The relationship between a sample spectrum, baseline spectrum, and the } \\
\text { normal/abnormal mass assignments }\end{array}$ & Partial \\
\hline 58 & $\begin{array}{l}\text { The relationship between a sample spectrum, contaminant spectrum, and } \\
\text { estimated concentration }\end{array}$ & No \\
\hline 59 & $\begin{array}{l}\text { Relationship between sample intensity spectrum, sample reference spectrum, and } \\
\text { sample absorption spectrum }\end{array}$ & No \\
\hline
\end{tabular}




\section{Display Design for Dempster's Rule of Combination}

Although an entire display was designed for the IDWMS based on the identified information requirements (Appendix A), the current discussion will focus on three graphic forms designed to represent the IDWMS implementation of Dempster's rule. These forms best exemplify the application of EID in this thesis. Similar graphic forms could not be designed for other IDWMS relationships, given time constraints and limited access to the system development process prevented the relationships from being studied at the level of detail needed to design consistent visual mappings. These less understood relationships include the relationship between conventional sensor readings, threshold values, and conventional mass assignments, as well as the relationship between the sample spectra, baseline spectra, and spectroscopic mass assignments.

Nevertheless, Dempster's rule of combination presents several interesting opportunities for display design. First, it is an example of the higher-order relationships that EID aims to identify and make directly perceptible to operators. Second, displaying this process information in such a fashion is a means to supporting appropriate trust (Lee, 2006; Lee \& See, 2004). Lastly, humans are poor at Bayesian reasoning (Tversky \& Kahneman, 1974) and researchers have designed displays to alleviate the biases to which human decision-makers succumb (Tsai, Miller, \& Kirlik, 2011; Burns, 2004). These previous works are relevant because the Dempster-Shafer theory is a generalization of Bayesian probability theory, and Dempster's rule of combination is a generalization of Bayes' law (Shafer, 1976). By visualizing Dempster's rule, the following designs aim to help operators supervise the IDWMS's fusion process such that they can identify situations that produce misleading results and adapt accordingly.

\subsection{Grid Display}

Burns and Hajdukiewicz (2004) suggested that textbooks can serve as a source of inspiration for graphic form design. Thus, one of the rule of combination displays is taken from Shafer (1976), who himself teaches the concept in terms of the visualization. For example, to describe the use of the normalization factor to ignore conflicting evidence, Shafer (1976) instructs readers to "discard all the rectangles thus committed to Ø [the empty set]" (p. 59). 
The IDWMS implementation of Dempster's rule was described and visualized in section 1.2. This description considered two sources of information: the IDWMS's conventional and spectroscopic analyses. In actuality, six sources of information exist, since each of the five conventional water parameters are compared to thresholds and produces a mass assignment function. In order to reduce these six pairs of normal-abnormal confidences to two pairs, the associate and commutative properties of Dempster's rule were leveraged to solve this problem. These properties allow the six mass assignment functions to be combined in any order. Thus, a solution was to first create two intermediate joint mass assignment functions from the original six. The ADS modelled the conventional and spectroscopic analyses as being two different information flows, suggesting that the five conventional mass assignment functions be fused into one (referred to as the conventional aggregate).

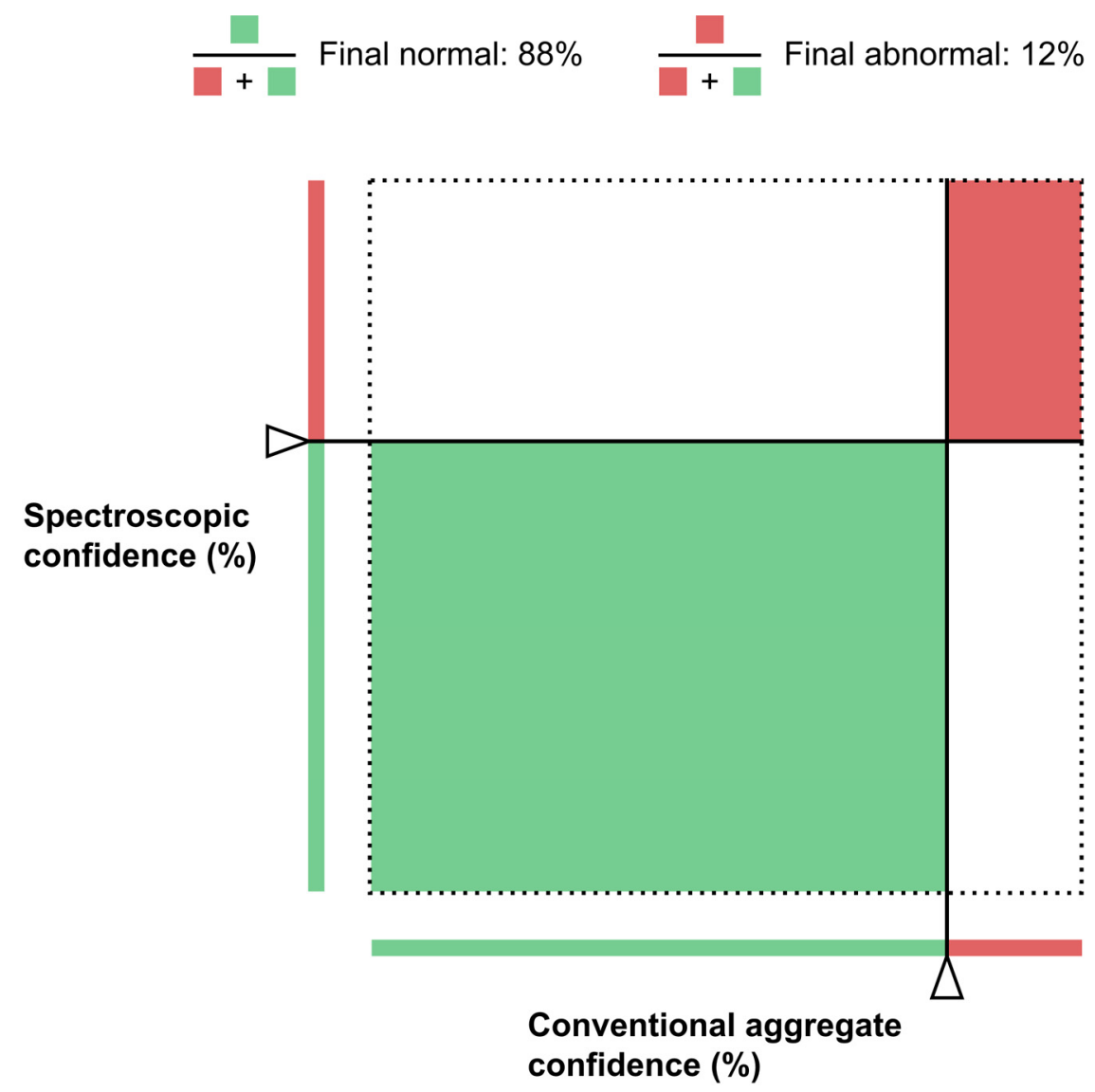

Figure 13. IDWMS rule of combination visualization 
The final graphic form designed for the IDWMS display is shown in Figure 13: green represents the belief that water is normal, while red represents the belief that water is abnormal. By mapping each individual mass assignment to a dimension of a rectangle, the product is represented by the area, creating an emergent feature. Together, these emergent features map to the rule of combination's solution: the joint belief in normal water is the proportion of green area to the total coloured area within the dotted square.

As noted in section 1.2.2, the IDWMS's simplified case of Dempster's rule is similar to Bayes' law. Burns (2004) designed a visualization for Bayes' law (Figure 14) which is similar to the described IDWMS display.

$$
P(K \mid k)=\frac{P(k \mid K) P(K)}{P(k \mid K) P(K)+[1-P(k \mid K)][1-P(K)]}
$$

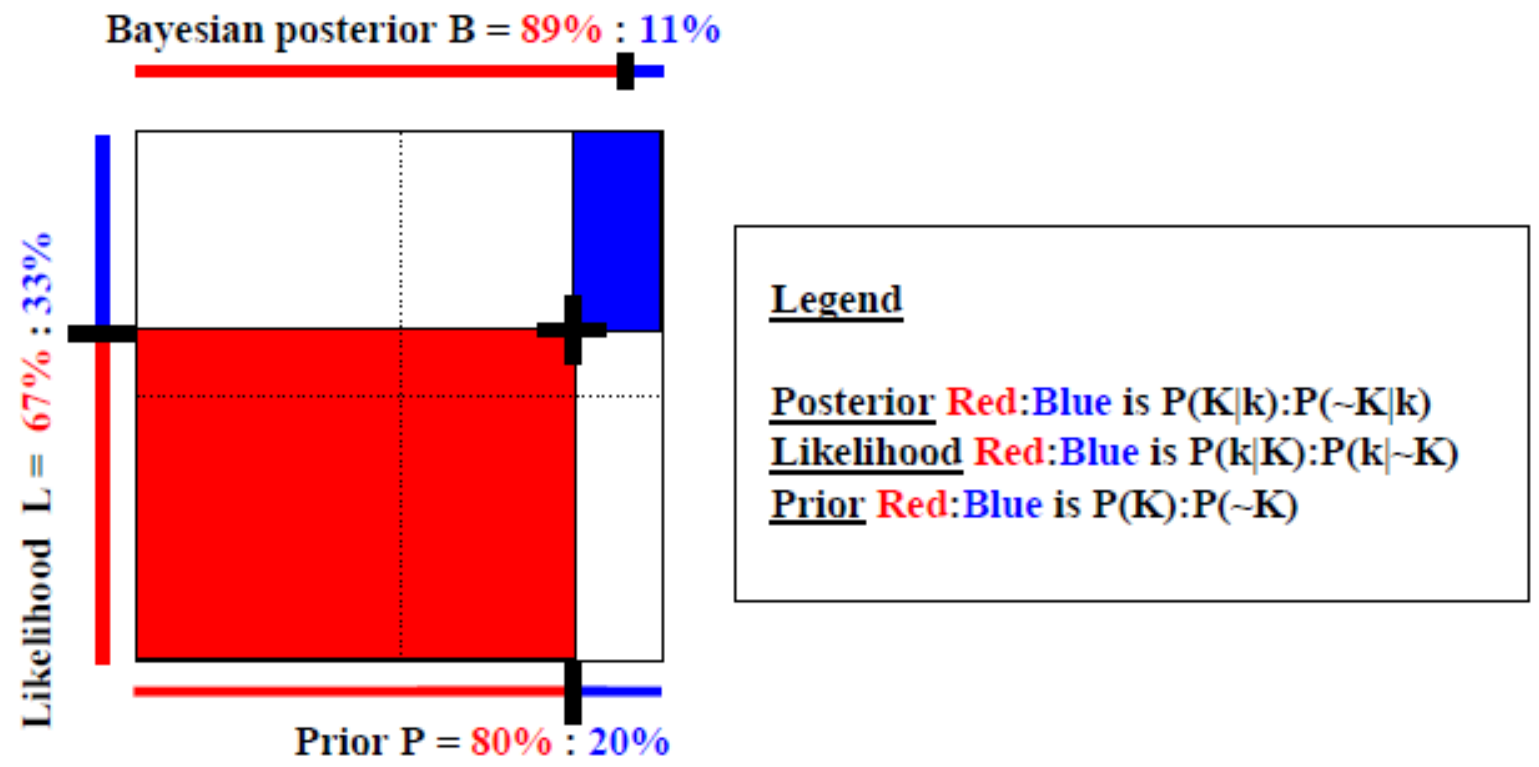

Figure 14. Visualization of Bayes' law (from Burns, 2004) 


\subsection{Trigonometric Display}

As an alternative to area, the multiplicative terms in Dempster's rule can be mapped to the trigonometric relationship $a=b \tan \theta$, where $\theta$ maps to length $c$ (Figure 15; Pawlak \& Vicente, 1996). The overall square is of a fixed size. To map $c$ to $\theta, c$ 's marker is connected to the right edge of the square. From this connection point, a diagonal is drawn to the lower-left corner of the square to create the angle $\theta$. The product $a$ is determined by extending $b$ 's marker upwards until it connects to the diagonal, forming a triangle with base $b$ and height $a$. Thus, the multiplicative relationship is mapped to the emergent feature of a triangle's shape. Since the IDWMS implementation of Dempster's rule of combination contains two unique product terms, the resulting design contains two such trigonometric forms (Figure 16). The joint mass assigned to abnormal water is the proportion of red to the total length of the stacked bar.

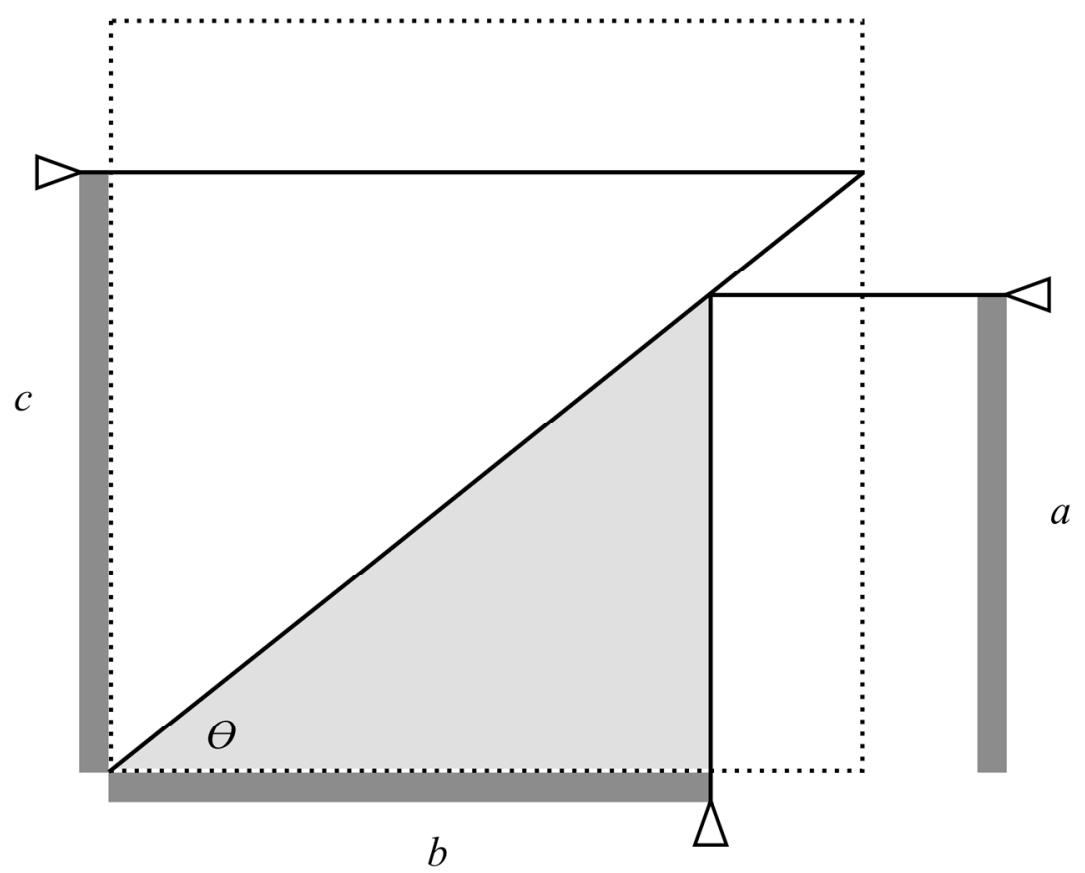

Figure 15. Trigonometric mapping for a multiplicative relationship 


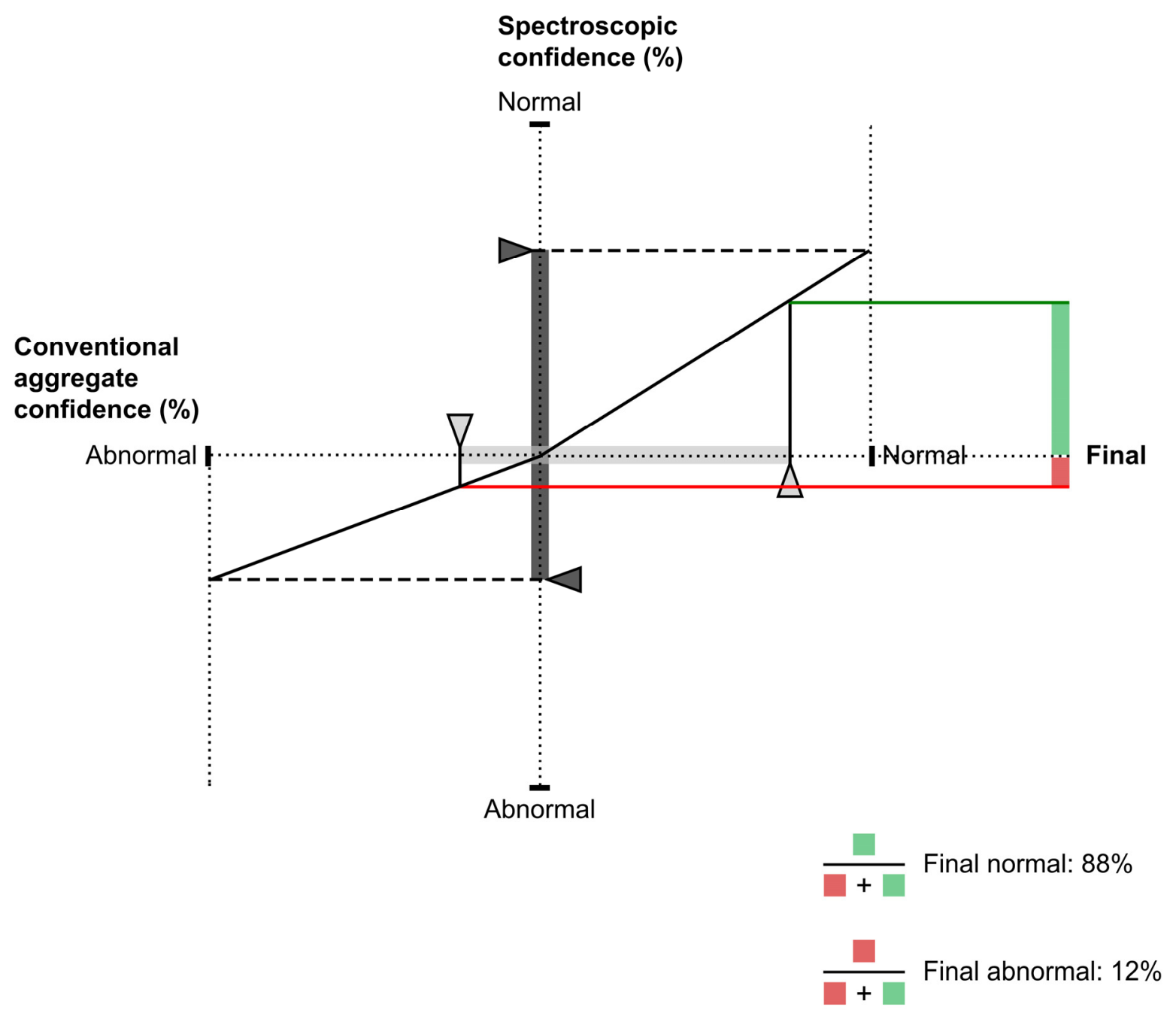

Figure 16. IDWMS trigonometric display for Dempster's rule of combination

Both the grid and trigonometric displays use emergent features to make Dempster's rule of combination directly perceptible. However, the grid display's emergent feature is expected to be a more effective representation for two reasons. First, Proximity Compatibility Principle research has found rectangles to be a particularly beneficial emergent feature (Barnett \& Wickens, 1988): a rectangle's area (representing some higher-level integrated measure) can be perceived without disrupting perception of its individual dimensions (representing lower-level information). Second, the trigonometric relationship is a more convoluted mapping because one variable (the spectroscopic mass assignment) must first be translated from a length to an angle before it can be integrated with the other variable (the conventional aggregate mass assignment). As a result, the intersection of propositions is less apparent in the trigonometric display. Burns and Hajdukiewicz (2004) have noted that training is needed to interpret this display. As a possible argument for the 
trigonometric display, Cleveland and McGill's (1984) psychophysical approach to display design found that angle is more accurately perceived than area. However, length is used in both displays and it was found to be more effective than angle. Furthermore, Cleveland and McGill (1984) did not investigate the effects of combining codes.

\subsection{Contour Plot Display}

An overview display was designed to communicate Dempster's rule of combination for several sensor arrays (Figure 17). The display is a contour plot of Dempster's rule of combination (Figure 18), with the $\mathrm{x}$ - and $\mathrm{y}$-axes representing the conventional aggregate and spectroscopic mass assignments, and the contour lines representing constant joint mass values (only three reference lines are displayed for readability). Thus, each plotted point represents all three mass assignments for a particular sensor array, resulting in a highly integrated display but no emergent feature, as the relationship between the three values for a given sensor site is not explicitly shown.

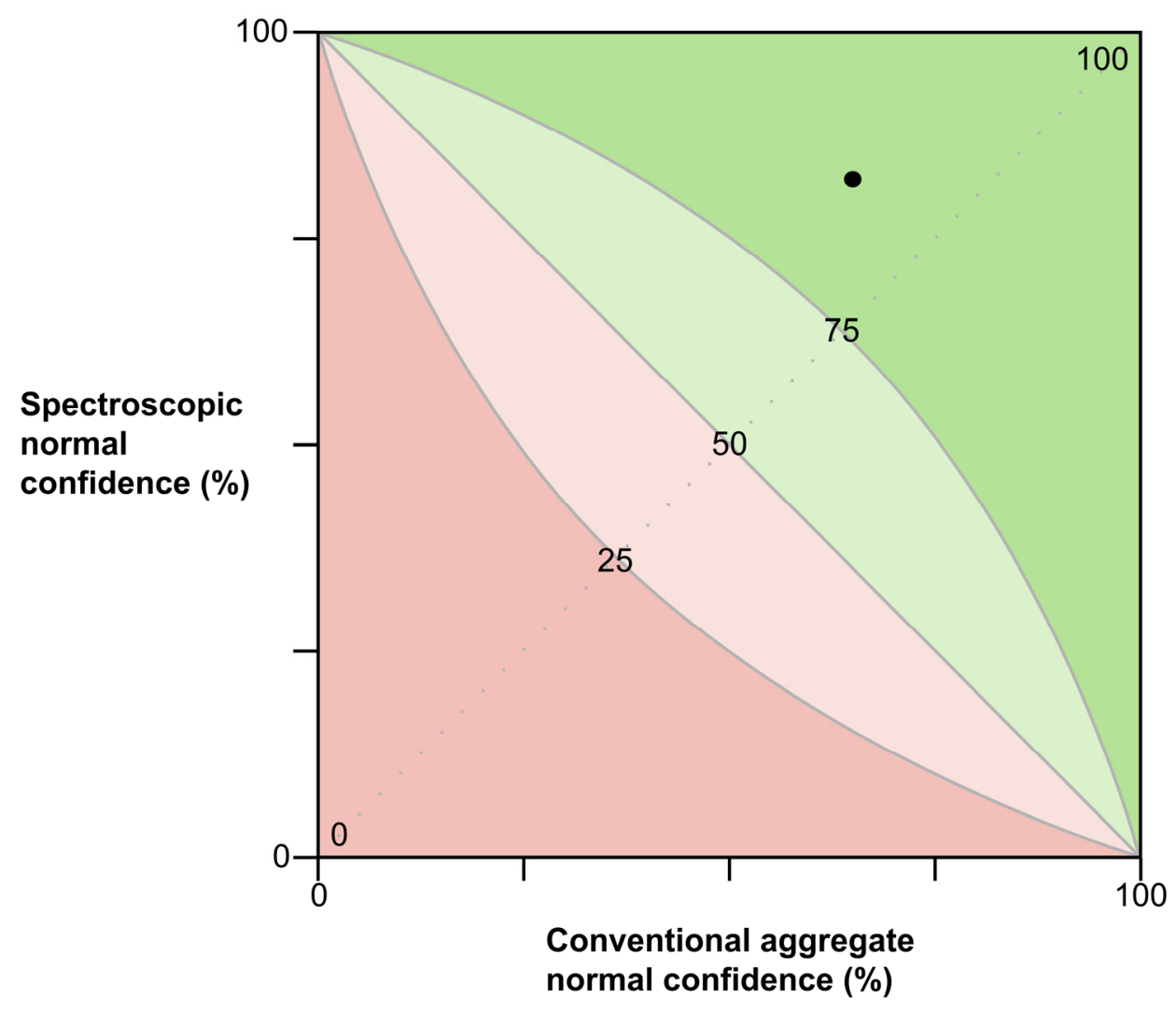

Figure 17. IDWMS contour plot for Dempster's rule 


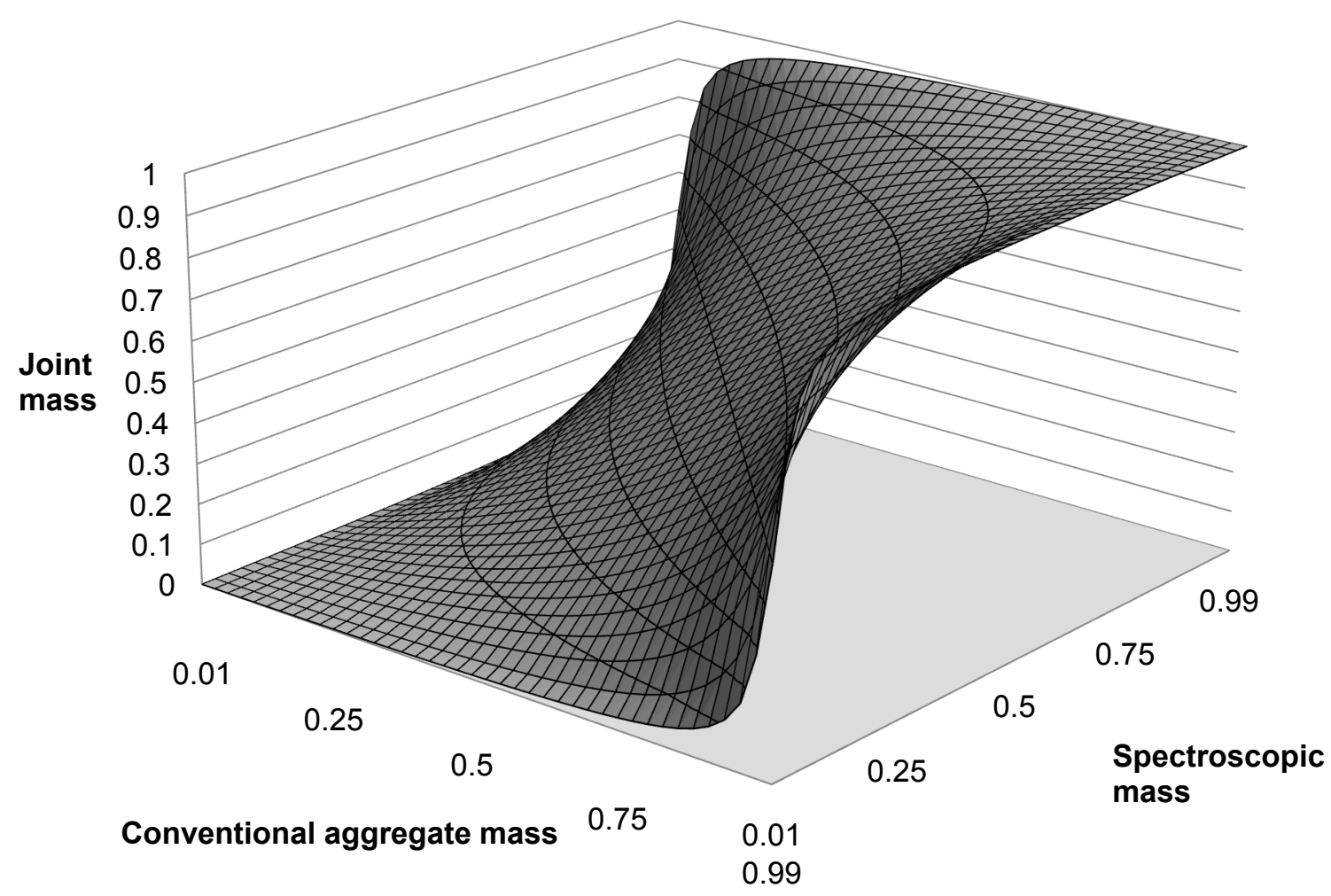

Figure 18. Three-dimensional plot of Dempster's rule 


\section{Display Evaluation}

A comparative display evaluation was conducted to investigate the effects of displaying a decision aid's underlying constraint. Participants performed a signal detection task using the decision aid, and effects were studied in terms of detection performance, as well as trust and reliance on the aid. The grid display presented in section 5.1 was chosen for evaluation because it establishes the most direct mapping to Dempster's rule. For comparison, a separated display that did not represent this relationship was created.

\subsection{Signal Detection Task}

The signal detection task in this current evaluation was adapted from Sorkin, Kantowitz, and Kantowitz (1976), who studied the effects of likelihood alarm displays on dual-task performance. Participants in Sorkin et al.'s (1976) study performed a signal detection task in conjunction with a manual tracking task. In this previous study, participants were presented with four randomly generated three-digit numbers (referred to here as cues). All four numbers were drawn from a normal distribution with a mean of 3 in no-signal trials and 4 in signal trials. The standard deviations of both distributions were equal. In each trial, the alarm reported the likelihood of a signal: for a likelihood between 0 to 0.25 , there was no alarm; between 0.25 to 0.50 , the alarm reported "possible signal"; between 0.50 to 0.75 , "likely signal"; between 0.75 to 1.00 , "urgent signal". To produce this signal likelihood, another random number was generated in the way as the four cues, but with a smaller standard deviation, such that the alarm was slightly more sensitive than the four cues combined. This alarm input value was not visible to participants. The signal likelihood reported by the alarm was calculated using the formula:

$$
P(\text { Signal })=\frac{P(\text { Statistic } \mid \text { Signal })}{P(\text { Statistic } \mid \text { Signal })+P(\text { Statistic } \mid \text { No-signal })}
$$

In the current experiment, participants were also presented with four cues generated using Sorkin et al.'s (1988) method. However, the no-signal and signal distribution means were 4 and 5, respectively, to reduce the occurrence of negative numbers. To use Dempster's rule, two alarm likelihoods were required. Thus, two alarm input values were generated using Sorkin et al.'s (1988) method, but with the revised no-signal and signal distribution means. These alarm 
likelihoods were used as the mass assignments for the proposition that a signal exists ${ }^{4}$. This mass was subtracted from 1 to obtain the mass for the no-signal proposition, since these were the only propositions for the detection task. Both mass assignment functions were then combined using Dempster's rule. To calculate signal detection theory measures, the aid's results were converted to a binary response using the rule employed by the IDWMS: if the signal proposition had a greater joint mass than the no-signal proposition, then the aid concluded "signal" (and vice versa). These three mass assignment functions, but not the alarm input values, were presented with the four cues to participants via the evaluated displays (described in section 6.3). In some trials, participants performed the task manually with only the four cues and no decision aid. For each trial, participants indicated whether they believed there was a signal or no-signal, and then rated their confidence in their decision.

The reliability of the decision aid's final conclusion was manipulated in the current study. Reliability was the probability that a signal existed given that the aid's final conclusion was "signal", which was equal to the probability that no signal existed given a "no-signal" final conclusion.

\subsection{Measuring Reliance}

To measure users' reliance on decision aids, several studies have used signal detection theory and its measure of response bias (Meyer, 2001; Maltz \& Meyer, 2001; Maltz \& Shinar, 2003; Neyedli, Hollands, \& Jamieson, 2011; Wang, Jamieson, \& Hollands, 2009). If the aid indicates that there is a signal in a given trial, then users relying on the aid should be more liberal in responding that a signal exists than if they performed the task manually. Conversely, users relying on the aid should be more conservative in responding "signal" when given no-signal feedback. Reliance is thus measured as changes in response bias, and the following equations can be adapted to measure users' reliance upon two aids with different reliabilities (Maltz \& Meyer, 2001; Maltz \& Shinar, 2003; Murrell, 1977; Wang et al., 2009):

$$
\begin{aligned}
& \text { Reliance on signal feedback }=\ln \beta_{\text {manual }}-\ln \beta_{\text {signal feedback }} \\
& \text { Reliance on no-signal feedback }=\ln \beta_{\text {manual }}-\ln \beta_{\text {no-signal feedback }}
\end{aligned}
$$

\footnotetext{
${ }^{4}$ Although probabilities and mass assignments are different concepts, the Dempster-Shafer theory supports the use of probabilities as mass assignments (Sentz, 2002).
} 
The natural logarithm of $\beta$ is used in these equations because $\beta$ itself is not an equal-interval measure: values are compressed between 0 and 1 when bias is liberal, while values range from 1 to infinity when bias is conservative (with 1 representing neutrality; See et al., 1997). In comparison, $\ln \beta$ is linear and takes negative values when bias is liberal, positive values when bias is conservative, and zero when bias is neutral. To calculate response bias based on observed hit and false alarm rates, the following equations are used:

$$
\begin{aligned}
& d^{\prime}=Z_{H}-Z_{F A} \\
& C=-0.5\left(Z_{H}+Z_{F A}\right) \\
& \beta=\exp \left(d^{\prime} \times C\right)
\end{aligned}
$$

While the above reliance equations use $\ln \beta$ as the measure of response bias, See, Warm, Dember, and Howe (1997) found C to be a more effective measure of bias. However, unlike C, an optimal value can be calculated for $\ln \beta$, against which observed reliance can be judged. For a two-stage detection system where the human is alerted by a binary warning system, optimal reliance depends on the conditional probability of the signal and no-signal states, given the output of the warning system (Meyer, 2001; Meyer \& Bitan, 2002; Murrell, 1977; Robinson \& Sorkin, 1985; Sorkin \& Woods, 1985). Thus, the optimal response bias values can be defined as (Murrell, 1977; Wang et al., 2009):

$$
\begin{aligned}
& \ln \beta_{\text {optimal for manual }}=\ln \left[\frac{P(\text { No-signal })}{P(\text { Signal })} \times \frac{V(C R)+C(F A)}{V(H)+C(\mathrm{M})}\right] \\
& \ln \beta_{\text {optimal for signal feedback }}=\ln \left[\frac{P(\text { No-signal } \mid \text { Signal feedback })}{P(\text { Signal } \mid \text { Signal feedback })} \times \frac{V(C R)+C(F A)}{V(H)+C(\mathrm{M})}\right] \\
& \ln \beta_{\text {optimal for no-signal feedback }}=\ln \left[\frac{P(\text { No-signal } \mid \text { No-signal feedback })}{P(\text { Signal| No-signal feedback })} \times \frac{V(C R)+C(F A)}{V(H)+C(\mathrm{M})}\right]
\end{aligned}
$$

However, the above equations apply to a binary warning system. In the current study, the aid's mass assignments for the signal and no-signal states were continuous. These were mapped to a binary result by taking the proposition with the greater joint mass to be the aid's final conclusion, but the mass assignment functions remained visible to participants. Thus, these equations do not consider the additional information provided by the continuous mass assignments. These equations are then better interpreted as describing effective reliance in the current study. In 
addition, since participants in the current study were instructed to weigh payoffs equally, the terms are not needed in the effective $\ln \beta$ calculations. Furthermore, the conditional probabilities in the following equations can be expressed in terms of aid reliability (as defined in section 6.1). The resulting equations for this study are:

$$
\begin{aligned}
& \ln \beta_{\text {optimal for manual }}=\ln \left[\frac{P(\text { No-signal })}{P(\text { Signal })}\right] \\
& \ln \beta_{\text {effective for signal feedback }}=\ln \left[\frac{P(\text { No-signal } \mid \text { Signal feedback })}{P(\text { Signal } \mid \text { Signal feedback })}\right]=\ln \left[\frac{1-\text { Aid reliability }}{\text { Aid reliability }}\right] \\
& \ln \beta_{\text {effective for no-signal feedback }}=\ln \left[\frac{P(\text { No-signal } \mid \text { No-signal feedback })}{P(\text { Signal } \mid \text { No-signal feedback })}\right]=\ln \left[\frac{\text { Aid reliability }}{1-\text { Aid reliability }}\right]
\end{aligned}
$$

As an alternative to signal detection theory, reliance can be studied using the concepts of automation misuse and disuse (Dzindolet, Peterson, Pomranky, Pierce, \& Beck, 2001; Dzindolet, Pierce, Beck, Dawe, \& Anderson, 2001). Misuse occurs when users engage automation in situations where it is inappropriate to do so (Parasuraman \& Riley, 1997). Misuse was measured as user error rates when the aid was incorrect. In cases of disuse, users ignore automation when it is beneficial to engage it (Parasuraman \& Riley, 1997). Disuse was measured as error rates when the aid was correct. Using this approach, users relying on automation will have greater misuse rates than disuse rates. However, no optimal level of misuse or disuse can be determined, so the appropriateness of reliance cannot be assessed. Both of the discussed approaches to assessing reliance were used in the present evaluation. Misuse and disuse allowed all of the detection performance data to be analyzed, whereas signal detection theory measures could not be calculated in cases where participants produced hit or false alarm rates of 1 or 0 . 


\subsection{Evaluated Displays}

In each trial, participants using the integrated display viewed the screen in Figure 19, which contained the grid display presented in section 5.1. A separate meter, labelled "Final", explicitly represented the joint mass assignments. The meter was divided into colour-coded regions to explicitly represent the presence of two propositions, as well as the translation from continuous mass assignments to a binary response. The height of the marker represented the mass assignment for the signal proposition. When this mass is greater than 0.5 , it will be greater than the complementary mass for the no-signal proposition, and the aid's final conclusion will be "signal". To indicate this, the region above 0.5 is red, as is the marker when inside this region. Likewise, the region and marker corresponding to signal mass assignments below 0.5 are coloured green to indicate the "no-signal" conclusion. This meter behaviour also applies to both intermediate mass assignment meters, labelled "A1" and "A2". Participants in the separated display group viewed three separate meters (Figure 20). 


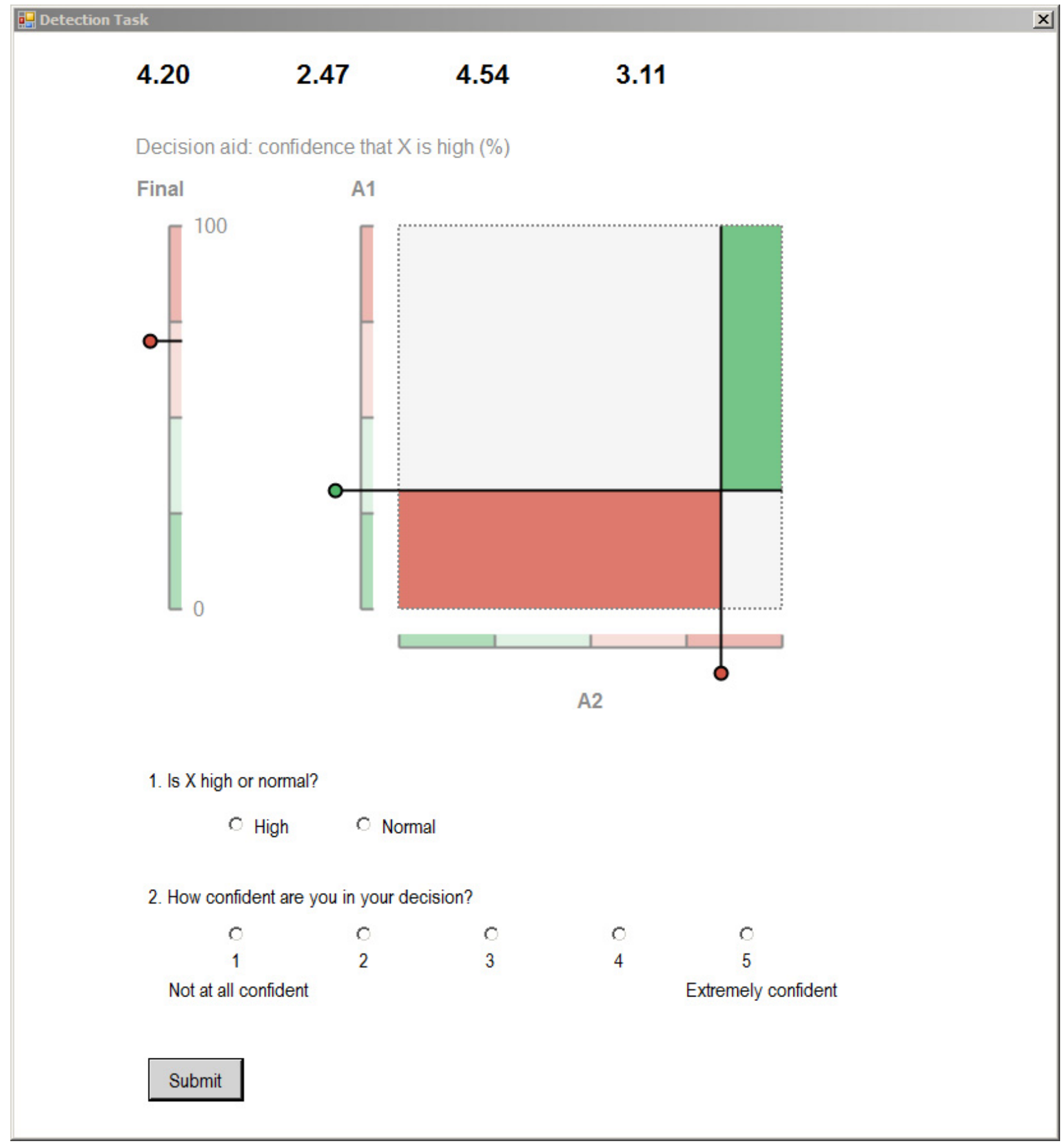

Figure 19. Experimental task screen with integrated display 


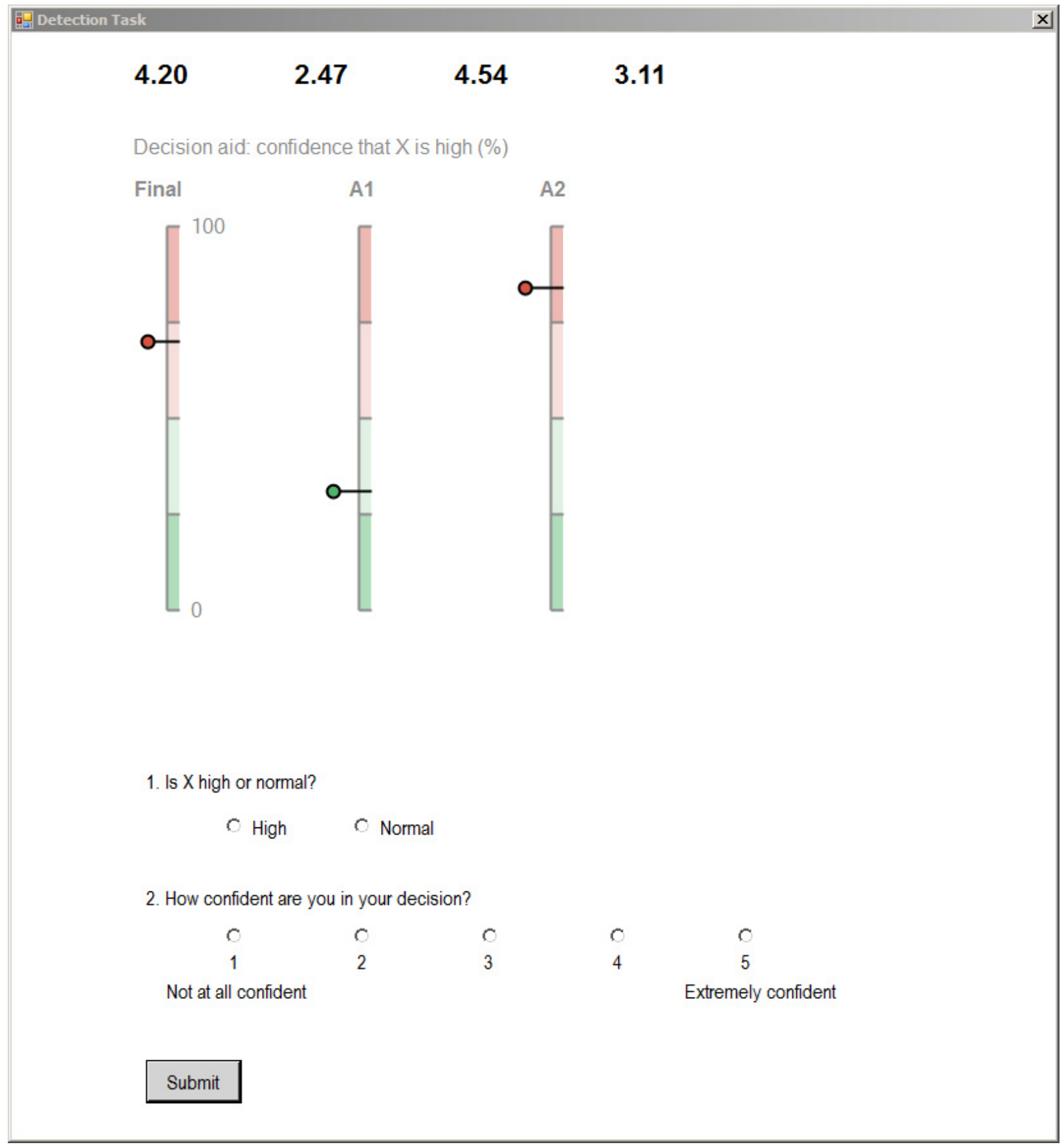

Figure 20. Experimental task screen with separated display

\subsection{Hypotheses}

The conducted study sought to investigate the effects of providing decision aid users with additional automation process information (i.e., Dempster's rule) via an object display. According to the trust in automation literature, providing users with automation process information should improve the appropriateness of trust (Lee \& See, 2004). However, trust is 
only one mediating factor of reliance. Another factor that seemed relevant is the cognitive overhead associated with engaging the automation (Parasuraman \& Riley, 1997). It was possible that the emergent features provided excessive information about the automation and overwhelmed users with visual information (Lee, 2006). Furthermore, the rectangles still required mental integration to produce the final result that was readily available in the Final meter. Additionally, participants were told that the final conclusion was more reliable than the A1 and A2 conclusions, and reliability disclosure has been found to affect aid reliance (Wang et al., 2009; McGuirl \& Sarter, 2006). Thus, participants may have chosen to rely primarily on the final conclusion and to disregard the intermediate results, especially since response time was measured.

The Proximity Compatibility Principle suggests that emergent features should support users in integrating information, but may impede access to lower-level codes (Wickens \& Carswell, 1995). However, rectangles have been found to support integration without this inhibition (Barnett \& Wickens, 1988). Given these conflicting possibilities, the evaluation tested for any differences in performance, reliance, and trust between users of the two display formats.

\subsection{Method}

A description of the experimental participants, design, measures, and procedure is provided in this section.

\subsubsection{Participants}

Thirty-six engineering students (whose years of university study had a mean of 2.6 and a standard deviation of 1.5) from the University of Toronto with no previous exposure to the Dempster-Shafer theory participated in the experiment. The sample size was selected based on reported effect sizes of display format on task performance (Carswell \& Wickens, 1996; Neyedli et al., 2011), as well as trust and reliance (Neyedli et al., 2011). Participants received $\$ 20$ for their participation in the single session.

\subsubsection{Experimental Design}

The experiment was a 3 (aid reliability level: no-aid, 80\%, 94\%) $\times 2$ (display: integrated, separated) mixed design, and used display as the between-subjects factor. In the no-aid condition, participants performed the detection task manually and were shown only the four cues (Figure 
21). Participants completed one separate block of trials for each reliability level, and the block order was counterbalanced. The proportion of signal and no-signal trials was equal in each block.

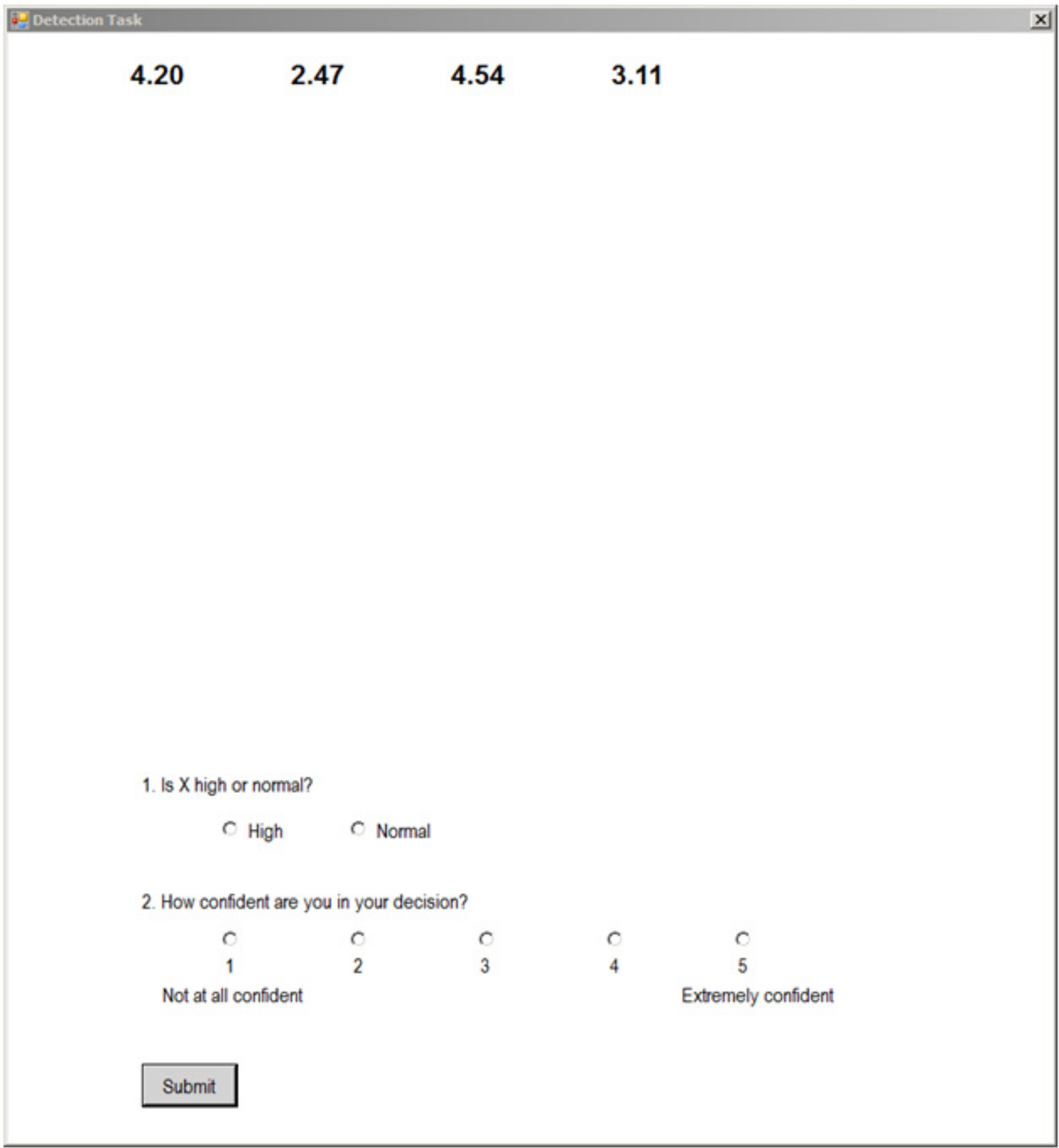

Figure 21. Experimental task screen in the no-aid (manual) condition 


\subsubsection{Measures}

Detection performance was measured as error rates (false alarms and misses), detection sensitivity, and response time. To assess reliance on the decision aid, response bias was measured using $\ln \beta$ and $\mathrm{C}$. Misuse and disuse rates were also used as indications of reliance (Dzindolet et al., 2001a). Lastly, Jian, Bisantz, and Drury’s (2000) empirically determined survey for assessing trust in automated systems was used to assess participants' trust in the decision aid (Appendix F). Two studies have validated this study (Safar \& Turner, 2005; Spain, Bustamante, \& Bliss, 2008).

\subsubsection{Procedure}

Upon arrival, participants listened to a brief introduction to the experiment and completed the informed consent form (Appendix B). Next, they reported their level of education, as well as previous exposure to the Dempster-Shafer theory (Appendix C). Training began with a brief reading about the detection task (independent of the decision aid) and 40 practice trials without the decision aid (Appendix D). Participants were told that half the trials in each block contained signals. In addition, participants were instructed to always treat the values of hits, misses, false alarms, and correct rejections as being equal. After each practice trial, participants were told if their response was correct or not.

Next, participants read about the decision aid and its display (Appendix D). This material explained the alarm input vales and resulting mass assignments (presented as the aid's "confidence") in general terms. Regarding the combination of mass assignments, participants were only told that the combination process ignored any disagreement between the two "basic" confidence values. To illustrate this point, several pairs of basic confidences that resulted in the same final confidence were presented. Participants were also told that the final conclusion was always more reliable than each of the basic conclusions. Lastly, those using the integrated display read a sentence explaining that the final signal confidence was the proportion of red area to total coloured area. Following the readings, participants completed a quiz to assess their comprehension of the training material. To conclude training, participants performed 40 practice trials with the decision aid (Appendix E). The reliability of the aid (80\%) was not disclosed for these trials and feedback was again given after each response.

Following training completion, participants performed three blocks of 100 trials, with each block being assigned one aid reliability level. Participants were instructed to complete these trials as 
quickly as possible without sacrificing accuracy. Immediately after completing a block with the decision aid, participants rated their trust in the aid for that particular block using Jian et al.'s (2000) trust questionnaire (Appendix F).

\subsection{Results}

To verify the applicability of parametric signal detection theory to the collected data, the assumption that the signal and noise distributions had equal variances was tested (Green \& Swets, 1966; Wickens \& Hollands, 2000). For each participant, normalized receiver operating characteristic points were calculated using participants' confidence ratings. Each participant had one set of points per combination of aid reliability and aid feedback (signal or no-signal). Least squares regression was performed on each set of points to find the slope of each normalized receiver operating characteristic curve. Due to the high aid reliability rates, many participants had hit and false alarm rates of 1 or 0 in some blocks, and data from these blocks were excluded from the analysis ${ }^{5}$. A two-tailed one-sample $t$-test revealed no significant difference between the obtained slopes and the assumed slope of $1, t(111)=-1.46, p=.15, M=.95, S E=.03$. This result indicated that the assumption of equal variances was met, permitting the use of d', $\beta$, and C.

\subsubsection{Performance}

Error rates were transformed using the following arcsine transformation to increase their normality (Howell, 2010):

\section{Transformed probability $=2 \arcsin \sqrt{\text { Probability }}$}

For the analyses of sensitivity and transformed false alarm rates, the Greenhouse-Geisser corrected degrees of freedom were used because the measures violated the assumption of sphericity.

\section{Detection Sensitivity, d'}

A 3 (aid reliability: no-aid, 80\%, 94\%) × 2 (display: separated, integrated) ANOVA performed on detection sensitivity found a main effect of reliability, $F(1.69,57.57)=102.46, p<.001, r=.87$

\footnotetext{
${ }^{5}$ One correction for such cases is to add and subtract half a hit or false alarm as needed (Macmillan \& Creelman, 2005). Such corrections were not used because of the small number of trials in which the aid was wrong, causing the half units to have a large effect on the signal detection measures. Furthermore, using this correction violated the assumption of equal variances, $t(177)=-2.01, p=.045, M=.94, S E=.03$.
} 
(Figure 22). Contrasts revealed significant differences between the no-aid and $80 \%$ reliability conditions, $F(1,34)=53.74, p<0.001, r=.78$, and between the $80 \%$ and $94 \%$ reliability conditions, $F(1,34)=75.19, p<0.001, r=.83$. Both display groups' detection sensitivity increased with aid reliability. No significant display effect was found, $F<1$, and no significant interaction effect was found, $F(1.69,57.57)=2.46, p=.10, r=.26$.

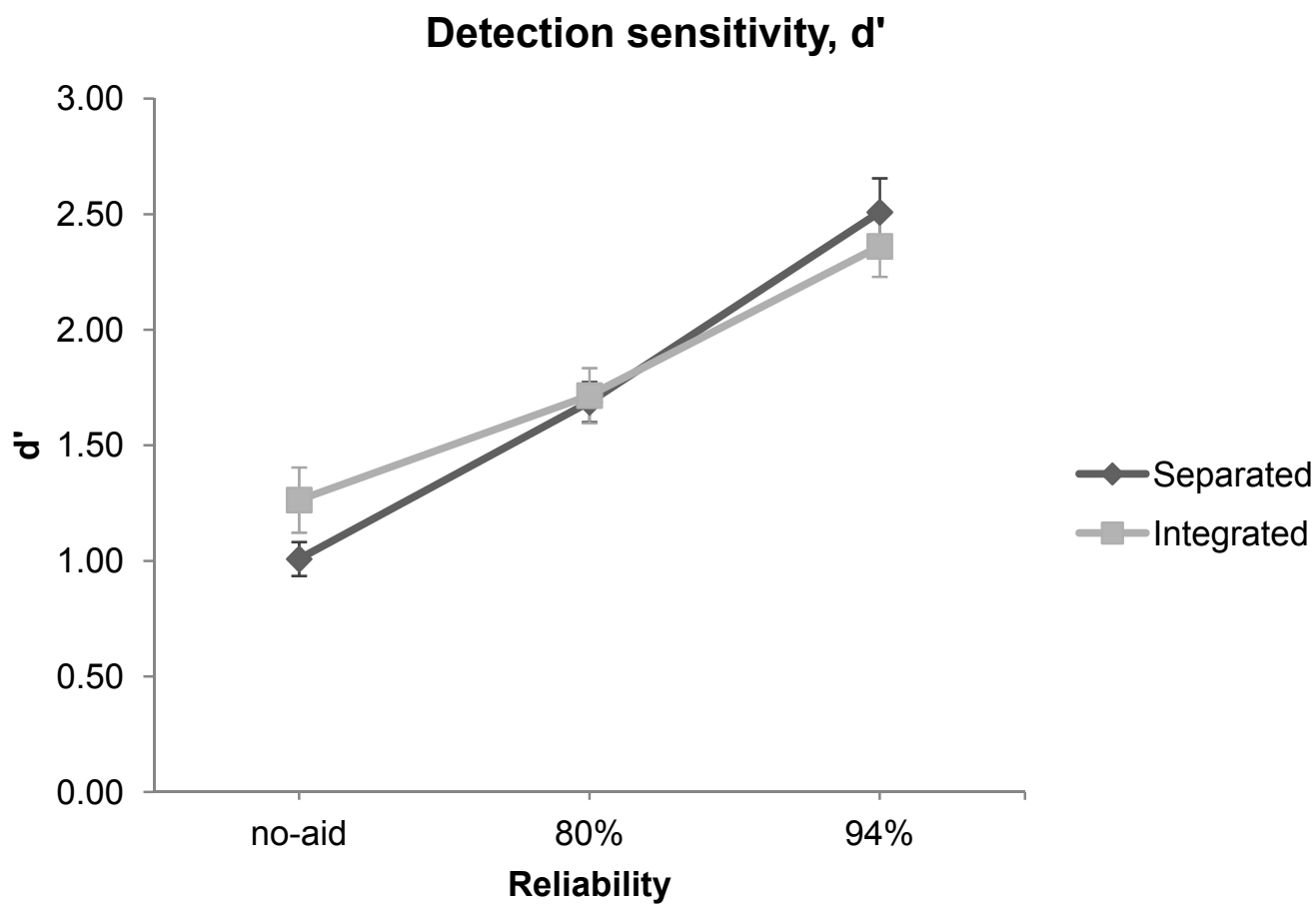

Figure 22. Effects of aid reliability and display on detection sensitivity, d'

\section{Miss Rate}

A 3 (aid reliability) $\times 2$ (display) ANOVA performed on the transformed miss rates also found a main effect of reliability, $F(2,68)=77.04, p<.001, r=.83$ (Figure 23). Both display groups produced fewer misses as aid reliability increased, as contrasts revealed significant differences between the no-aid and $80 \%$ reliability conditions, $F(1,34)=26.35, p<.001, r=.67$, and between the $80 \%$ and $94 \%$ reliability conditions, $F(1,34)=69.38, p<.001, r=.82$. The interaction between reliability and display was also significant, $F(2,68)=5.84, p<.01, r=.38$. Contrasts showed no significant difference between the no-aid and $80 \%$ reliability conditions, $F(1,34)=2.32, p=.14, r=.25$, but a significant difference between the $80 \%$ and $94 \%$ reliability conditions, $F(1,34)=4.84, p<.05, r=.35$. Integrated display users committed fewer misses 
than the separated display users when the aid was $80 \%$ reliable, but the reverse was true when the aid was $94 \%$ reliable. Display did not have a significant effect, $F<1$.

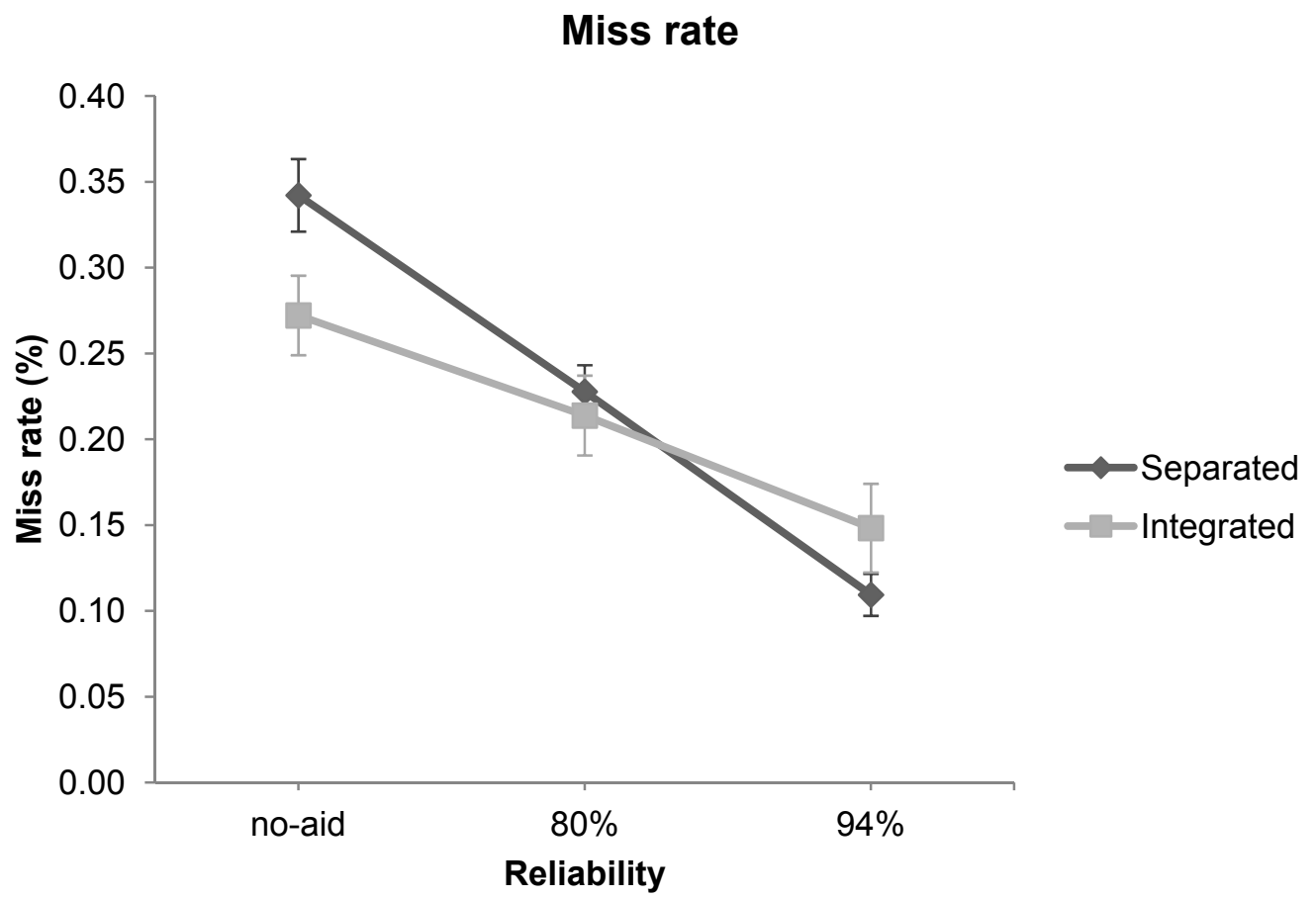

Figure 23. Effects of aid reliability and display on miss rate

\section{False Alarm Rate}

The transformed false alarm rates were also studied using a 3 (aid reliability) $\times 2$ (display) ANOVA, which found a significant effect for reliability, $F(1.68,57.02)=77.04, p<.001, r=.79$ (Figure 24). Contrasts found significant differences between the no-aid and $80 \%$ reliability conditions, $F(1,34)=38.82, p<.001, r=.73$, and between the $80 \%$ and $94 \%$ reliability conditions, $F(1,34)=34.15, p<.001, r=.71$. Both display groups produced fewer false alarms as aid reliability increased. Neither the display effect nor interaction effect were significant, $F<$ 1 in both cases. 


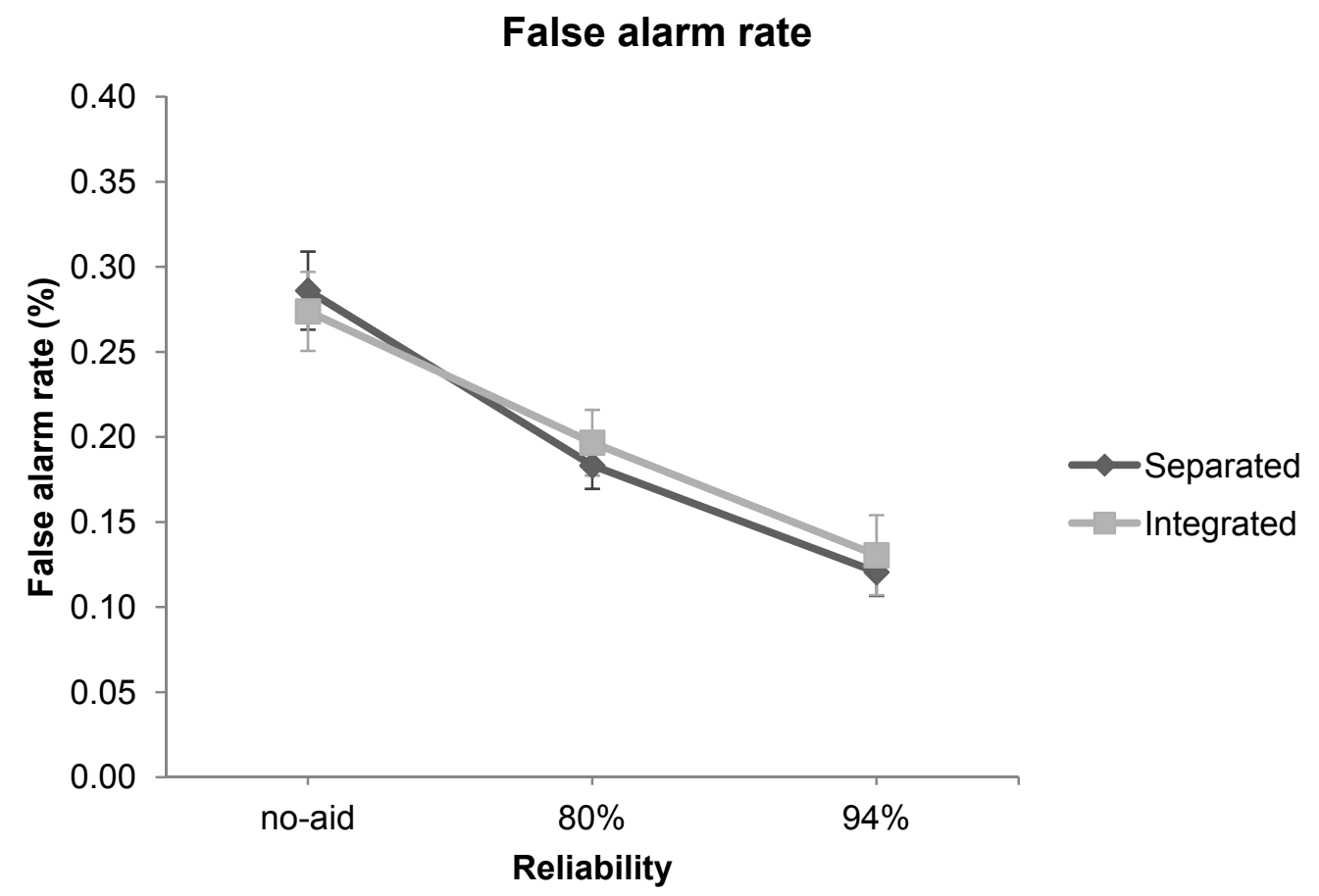

Figure 24. Effects of aid reliability and display on false alarm rate

\section{Response Time}

The square root was taken for response times to normalize the data for analysis. From the 3 (aid reliability) $\times 2$ (display) ANOVA performed on the transformed data, no significant main or interaction effects were found, $F<1$ in each case (Figure 25). 


\section{Response time}

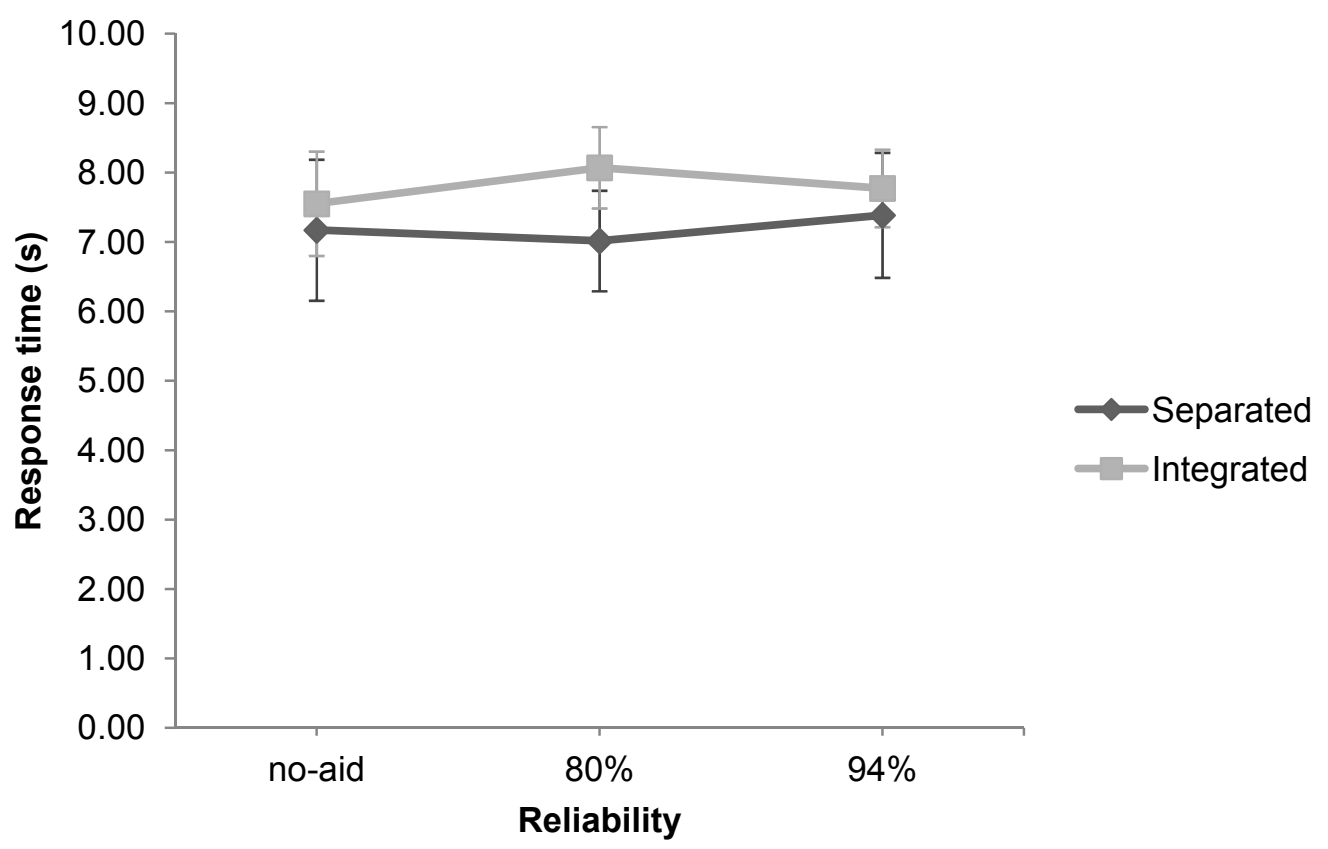

Figure 25. Effects of aid reliability and display on response time

\subsubsection{Reliance}

Since reliance is measured as changes in response bias, the three reliability levels were analyzed as reliability differences: no-aid - 80\%, $80-94 \%$, and no-aid - 94\%. However, observations for the no-aid -94\% condition are dependent on the two other reliability differences, and only the no-aid $-80 \%$ and $80-94 \%$ conditions were submitted to ANOVAs.

\section{$\ln \beta$ difference}

A 2 (aid reliability difference: no aid $-80 \%, 80-94 \%$ ) $\times 2$ (aid feedback: high, normal) $\times 2$ (display: separated, integrated) ANOVA performed on $\ln \beta$ differences revealed a main effect of feedback, $F(1,11)=89.22, p<.001, r=.94$ (Figure 26 and Figure 27). Participants became more liberal when given signal feedback and more conservative when given no-signal feedback, which is indicative of reliance. No main effects of reliability difference or display were found, nor was a three-way interaction found; $F<1$ in all cases. No significant two-way interactions were found: for feedback $\times$ display, $F(1,11)=3.73, p=.08, r=.50$, and for difference $\times$ feedback, $F(1,11)=1.84, p=.20, r=.38$. 


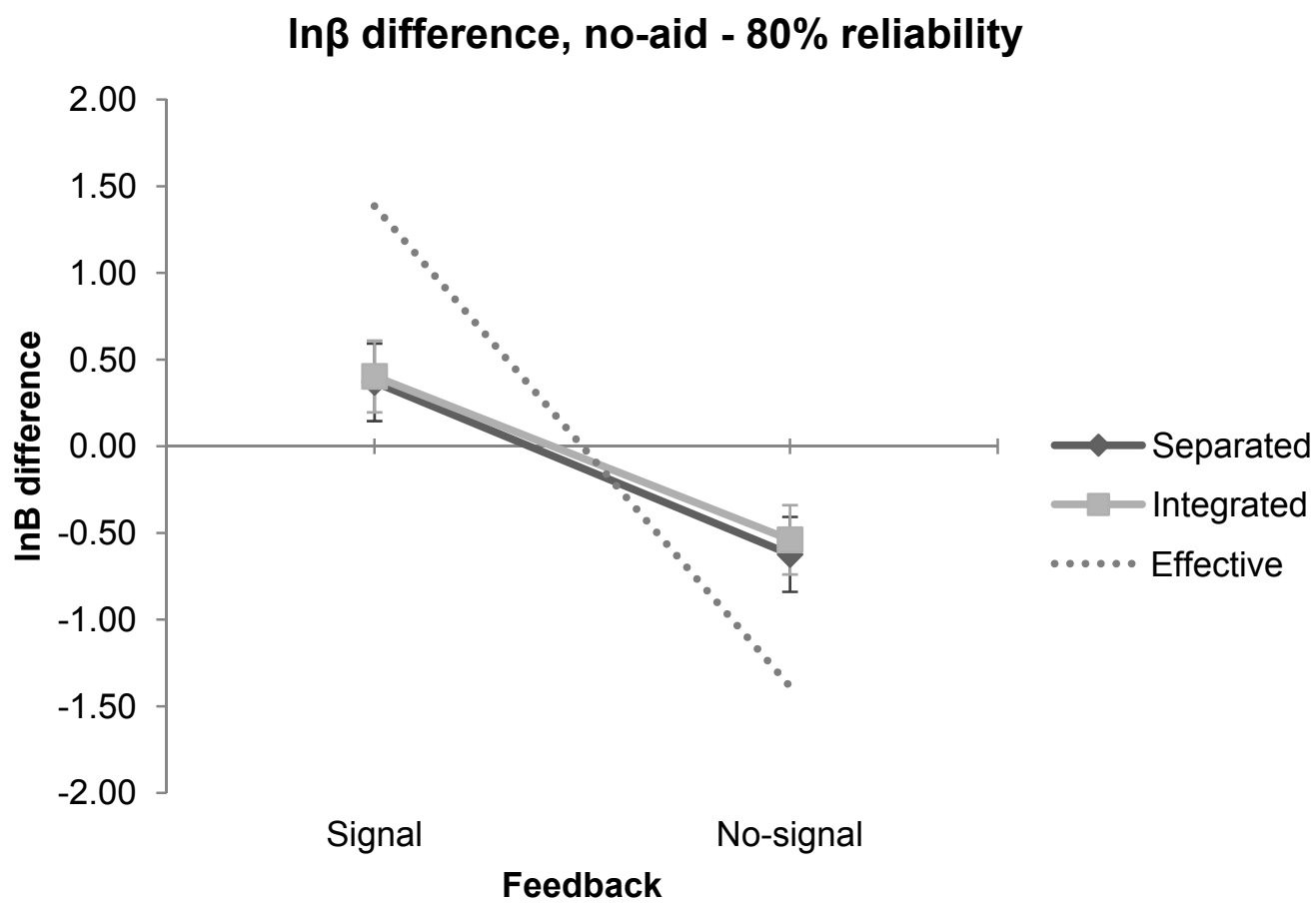

Figure 26. Effects of aid feedback and display on $\ln \beta$ differences for the no-aid $-80 \%$ reliability difference

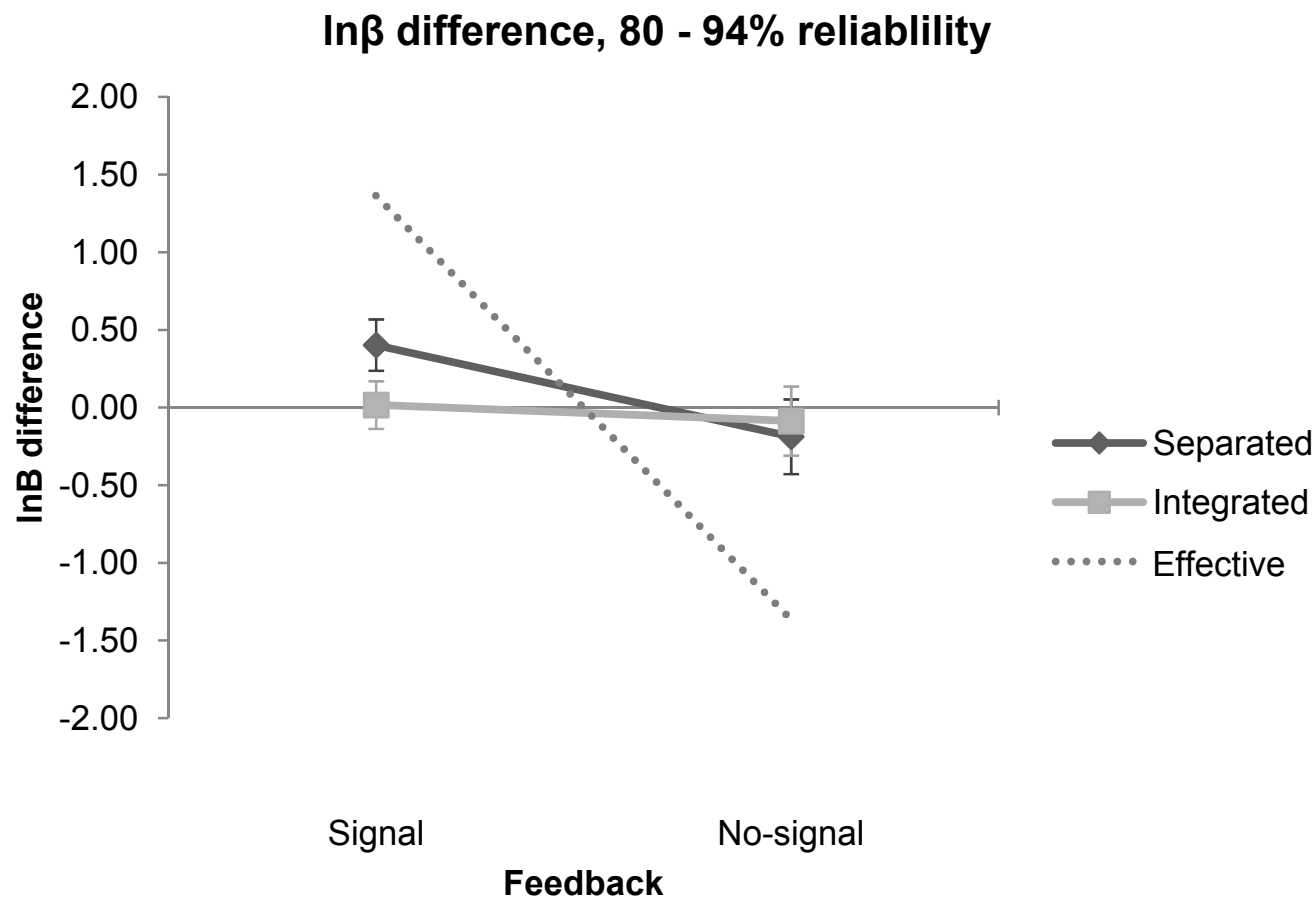

Figure 27. Effects of aid feedback and display on $\ln \beta$ differences for the $80 \%-94 \%$ reliability difference 
A series of one-sample two-tailed $t$-tests were performed to compare participants' $\ln \beta$ shifts with the effective values. Given signal feedback, the separated display yielded $\ln \beta$ decreases that were significantly less than effective for all three reliability difference conditions (no aid - 80\%, $80-$ $94 \%$, and no aid -94\%): $t(16)=-3.92, p<.01, r=.70 ; t(8)=-7.21, p<.001, r=.93 ; t(8)=-$ 18.81; $p .001, r=.99$, as did the integrated display: $t(13)=-5.63, p<.001, r=.84 ; t(9)=-6.67$, $p<.001, r=.91 ; t(9)=-8.93, p<.001, r=.95$ (Figure 28).

\section{In $\beta$ difference, signal feedback}

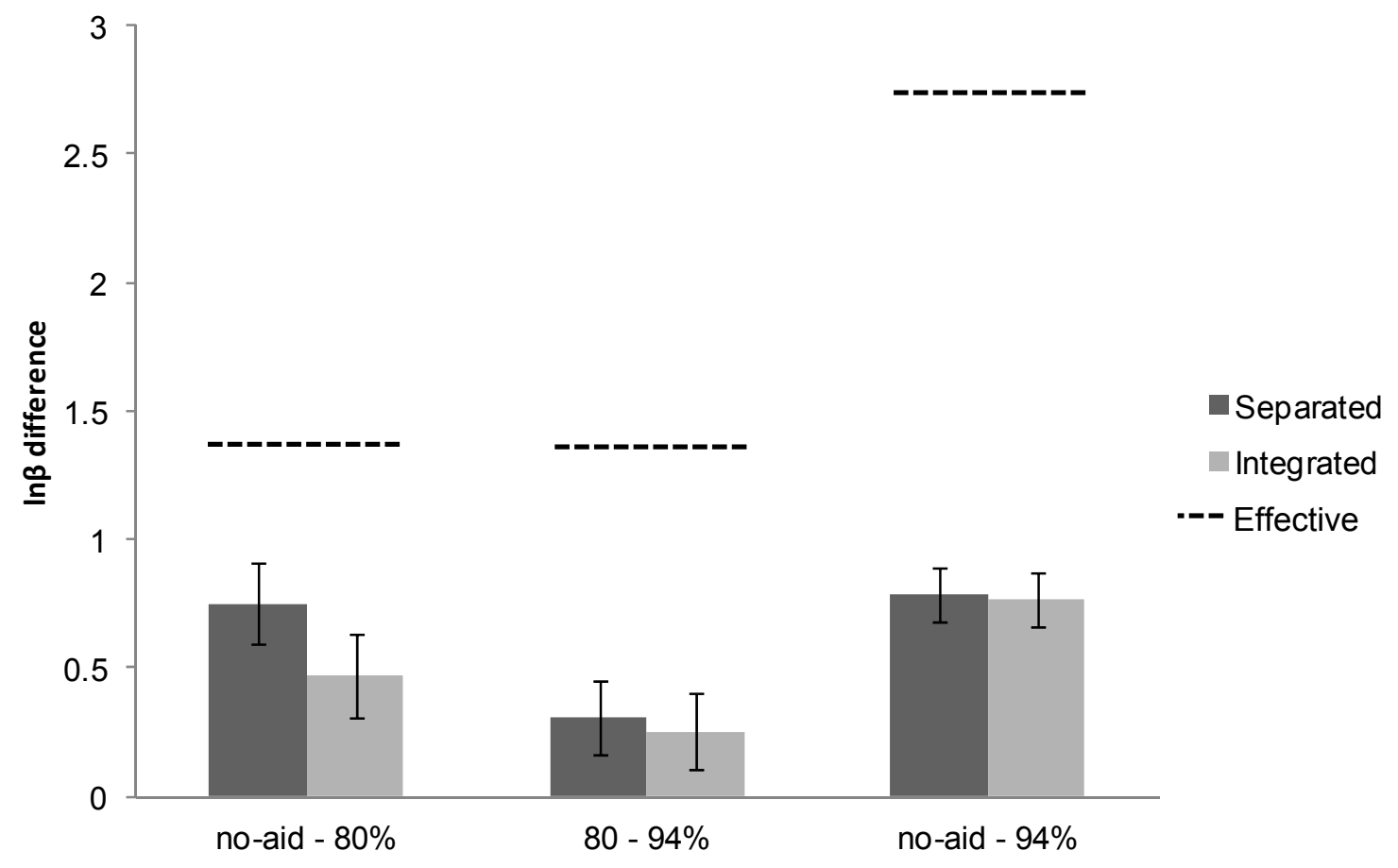

Figure 28. One-sample two-tailed $t$-tests comparing observed and effective $\ln \beta$ differences for signal feedback

The same sub-effective reliance was observed in the no-signal feedback: for the separated display, $t(15)=5.75, p<.001, r=.83 ; t(7)=5.04, p<.01, r=.88 ; t(7)=9.99, p<.001, r=.96$, and for the integrated display, $t(15)=4.19, p<.01, r=.73 ; t(9)=13.55, p<.001, r=.98 ; t(9)=$ $10.82, p<.001, r=.96$ (Figure 29). 


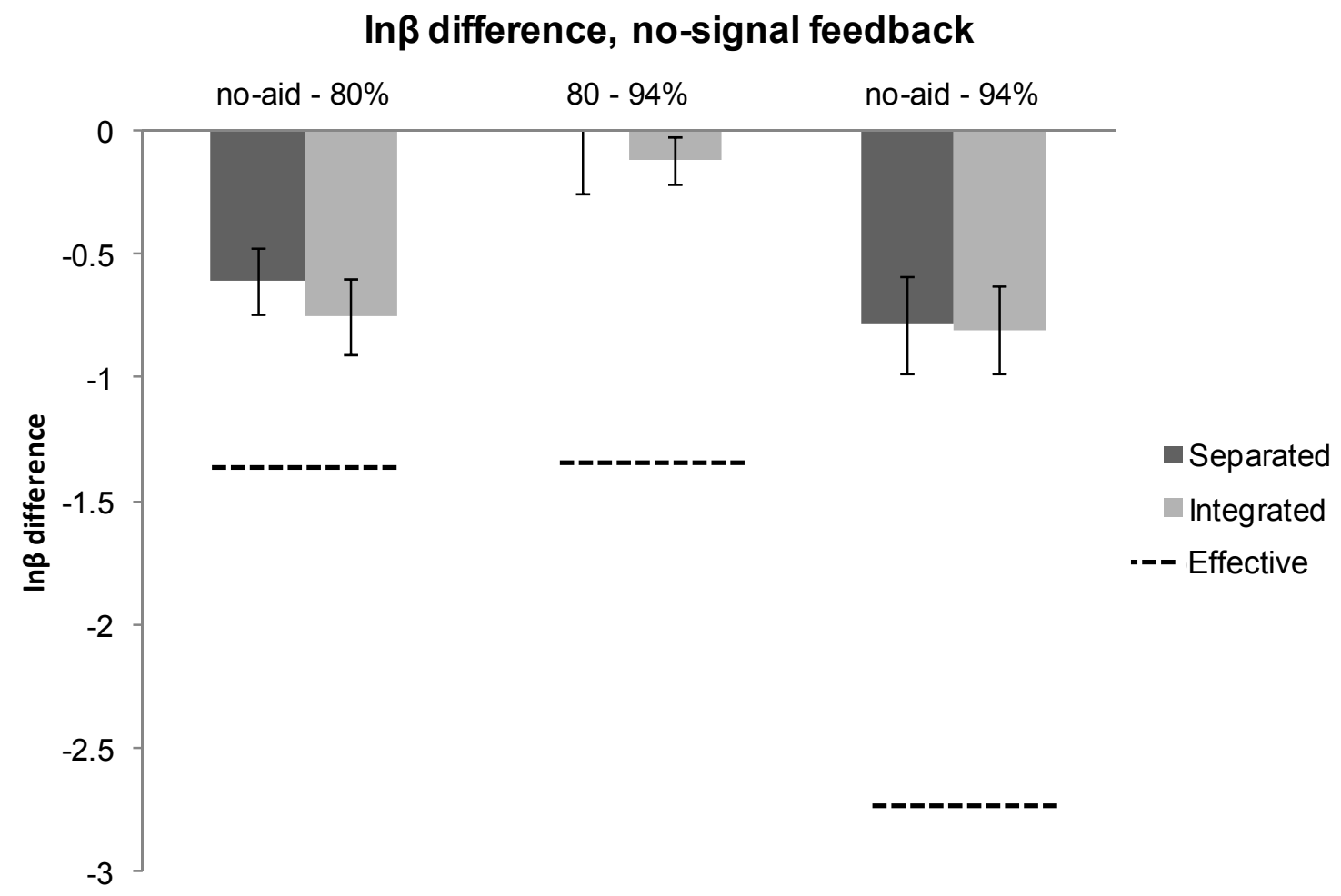

Figure 29. One-sample two-tailed $t$-tests comparing observed and effective $\ln \beta$ differences for signal feedback

\section{Difference}

Like the $\ln \beta$ analysis, $C$ differences were studied using a 2 (aid reliability difference) $\times 2$ (aid feedback) $\times 2$ (display) ANOVA, which found a significant effect for feedback, $F(1,11)=$ $159.647, p<.001, r=.97$. The effect of feedback on $\mathrm{C}$ differences is consistent with those observed with $\ln \beta$ differences: participants became more liberal when the decision aid provided signal feedback, and more conservative given no-signal feedback. Unlike the $\ln \beta$ comparison, a significant interaction between feedback and display was found, $F(1,11)=8.60, p=.01, r=.66$. In both reliability difference conditions, the separated display users showed greater decreases in $\mathrm{C}$ when given signal feedback, and greater increases in $\mathrm{C}$ when given no-signal feedback (Figure $30)$.

In addition, a significant interaction between feedback and reliability difference was found, $F(1$, $11)=5.23, p=.04, r=.57$. For both displays, participants reduced $\mathrm{C}$ more dramatically in the no-aid $-80 \%$ condition than the $80-94 \%$ condition when given signal feedback. The no-aid $80 \%$ condition also yielded greater increases in $\mathrm{C}$ when given no-signal feedback (Figure 31 ). 
No main effect of reliability difference was found, $F(1,11)=2.58, p=.14, r=.43$. No significant effects were found for the main display effect, the reliability difference $\times$ display interaction, or the three-way interaction; $F<1$ in all cases.

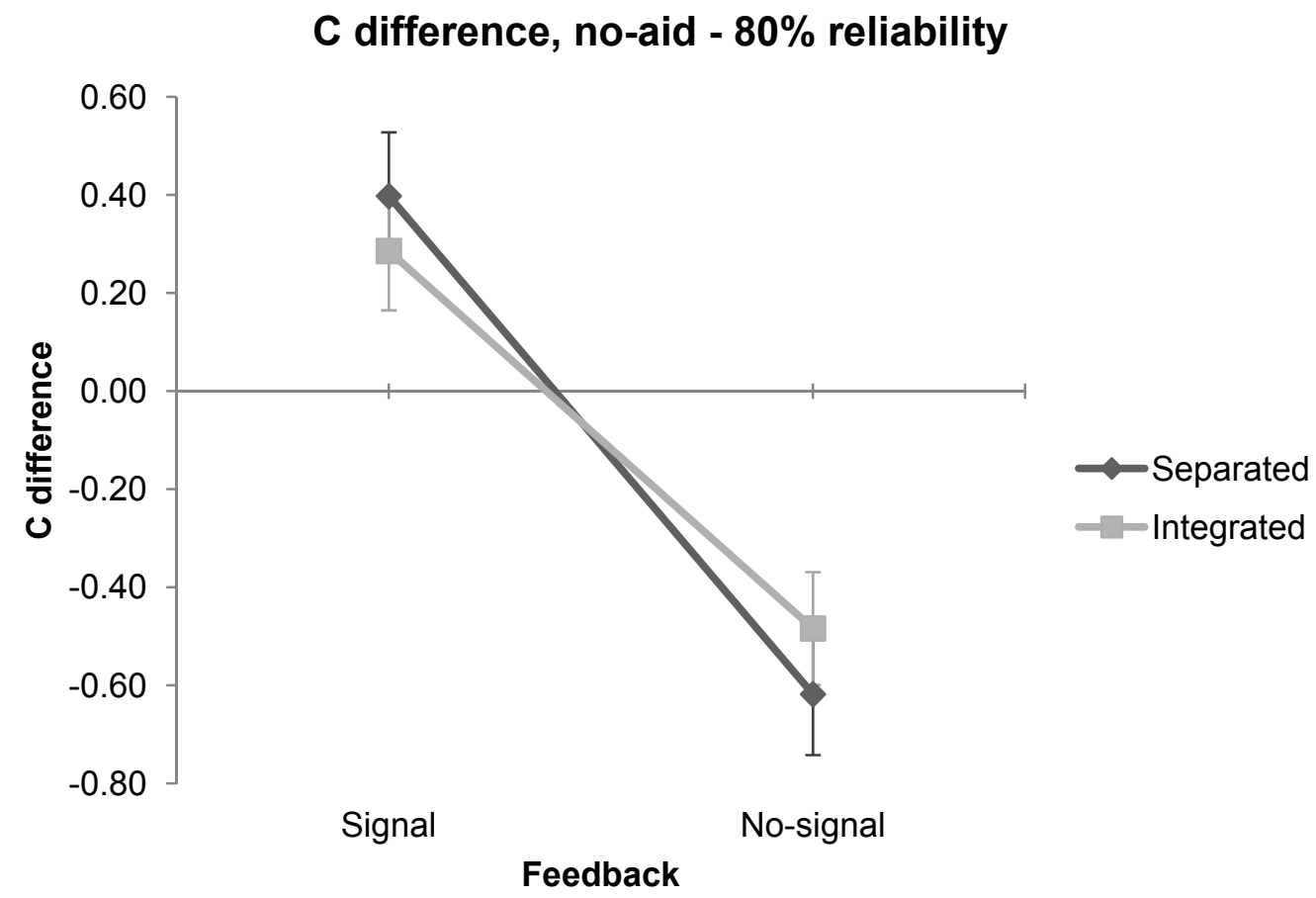

Figure 30. Effects of aid feedback and display on $\mathrm{C}$ differences for the no-aid $-80 \%$ reliability difference 


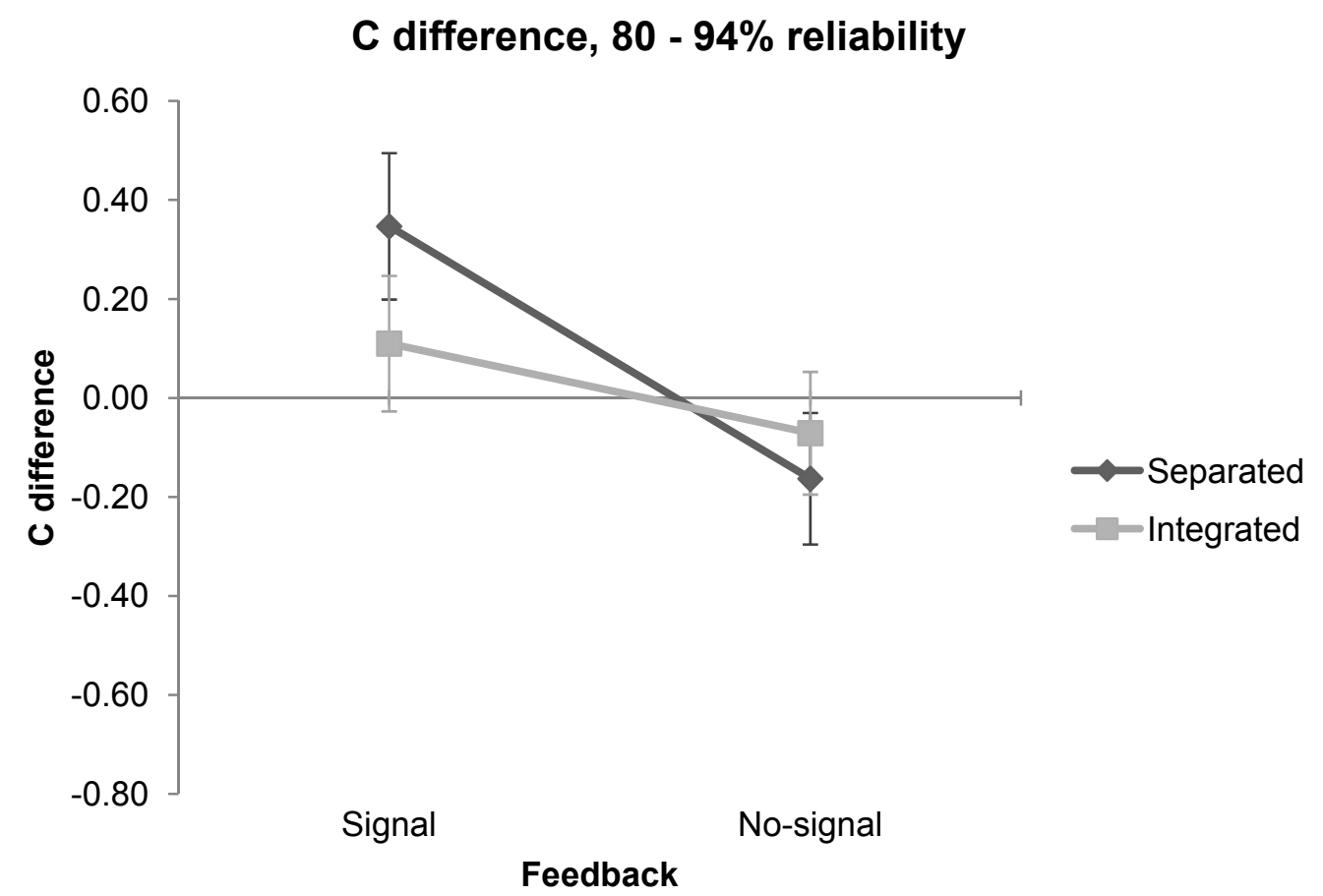

Figure 31. Effects of aid feedback and display on C differences for the $80 \%-94 \%$ reliability difference

\section{Misuse and Disuse}

Dzindolet et al.'s (2001a) measures of misuse (errors committed when the aid is incorrect) and disuse (errors committed when the aid is correct) allowed all of the collected detection performance data to be analyzed. A 2 (aid reliability: 80\%,94\%) $\times 2$ (aid result: correct, incorrect) $\times 2$ (display: separated, integrated) ANOVA performed on transformed error rates found a significant effect of aid result, $F(1,34)=76.32, p<.001, r=.83$ (Figure 32 and Figure 33). For both displays and aid reliability levels, participants committed fewer errors when the aid was correct than when the aid was incorrect. That is, there was a higher rate of misuse than disuse, indicating that both display groups relied upon the aid. The interaction between aid result and reliability was also significant, $F(1,34)=8.63, p<.01, r=.45$. For both displays, participants committed more errors when the aid was correct in the $80 \%$ reliability condition compared to the $94 \%$ reliability condition. Participants also committed fewer errors when the aid was wrong in the $80 \%$ reliability condition, indicating greater reliance in the $94 \%$ reliability condition. Reliability did not have a significant effect, $F(1,34)=1.26, p=.27, r=.19$, nor did the reliability $\times$ display, aid result $\times$ display, and three-way interactions $(F<1$ in each case). 


\section{Separated display error rate}

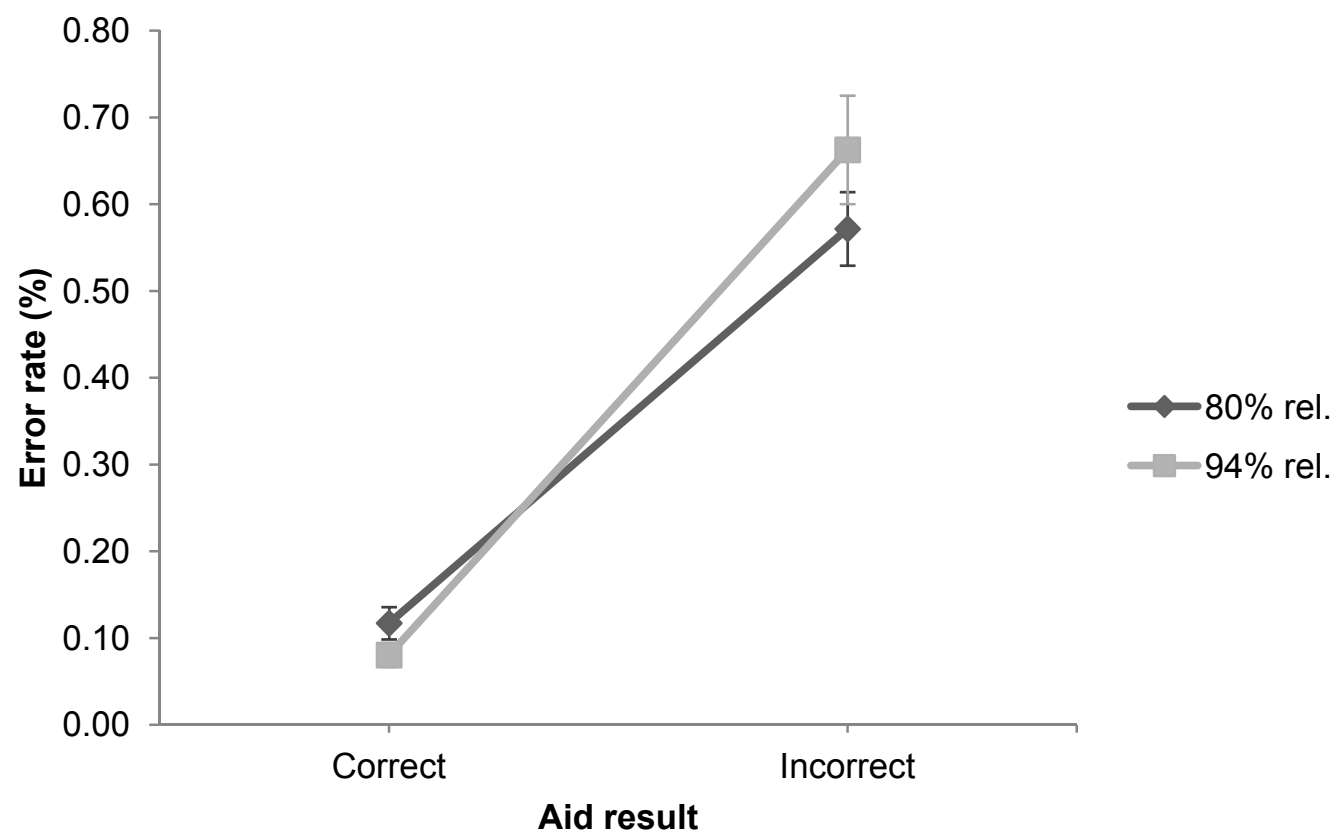

Figure 32. Effects of aid result and reliability on error rate for the separated display. Errors committed when the aid is correct indicate disuse.

Errors committed when the aid is incorrect indicate misuse.

Integrated display error rate

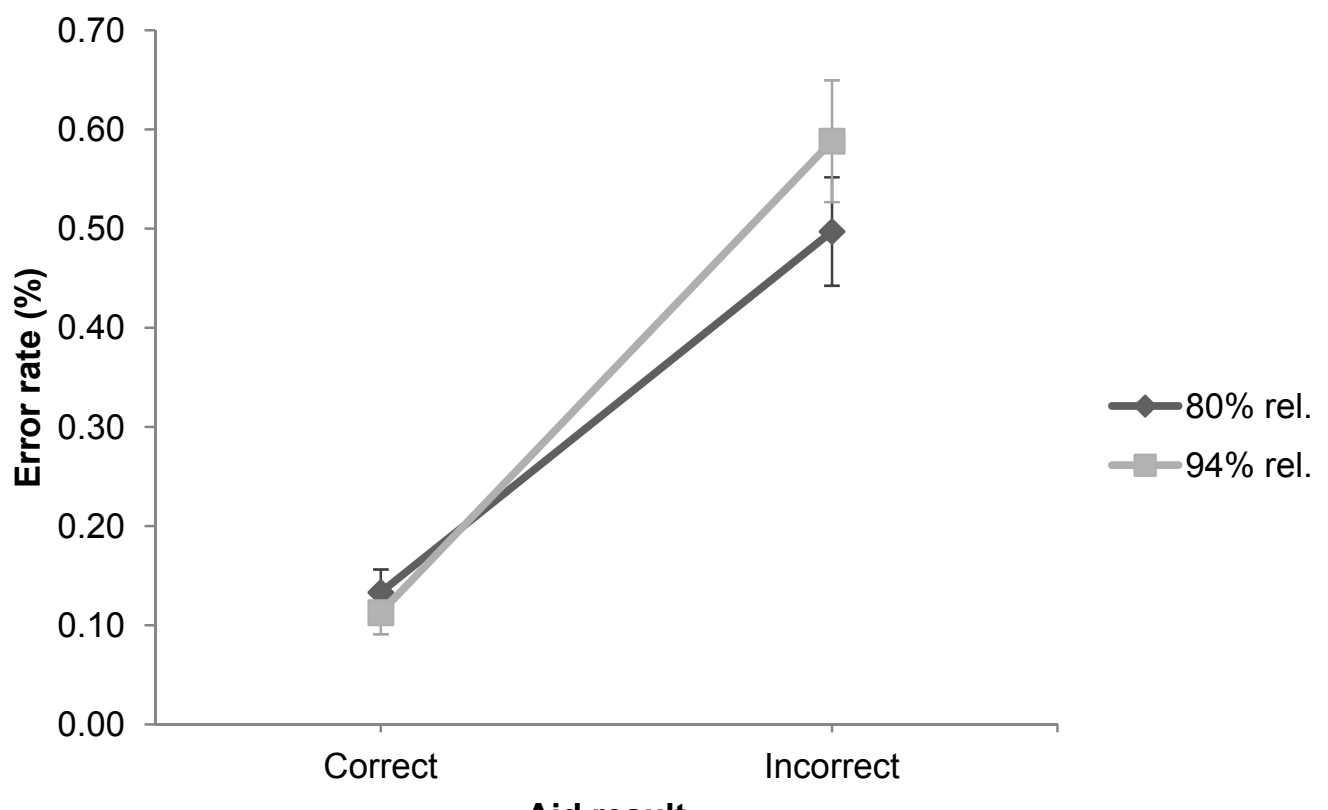

Aid result

Figure 33. Effects of aid result and reliability on error rate for the integrated display 


\subsubsection{Trust}

A 2 (aid reliability: 80\%, 94\%) $\times 2$ (display: separated, integrated) ANOVA on participants' mean trust ratings (calculated using all 12 questionnaire items) revealed a main effect of reliability, $F(1,34)=11.08, p<.01, r=.50$ (Figure 34). Participants indicated increased trust in the decision aid when it was more reliable. The main effect of display was not significant, $F(1$, $34)=1.39, p=.25, r=.20$, nor was the interaction effect, $F<1$.

\section{Trust rating}

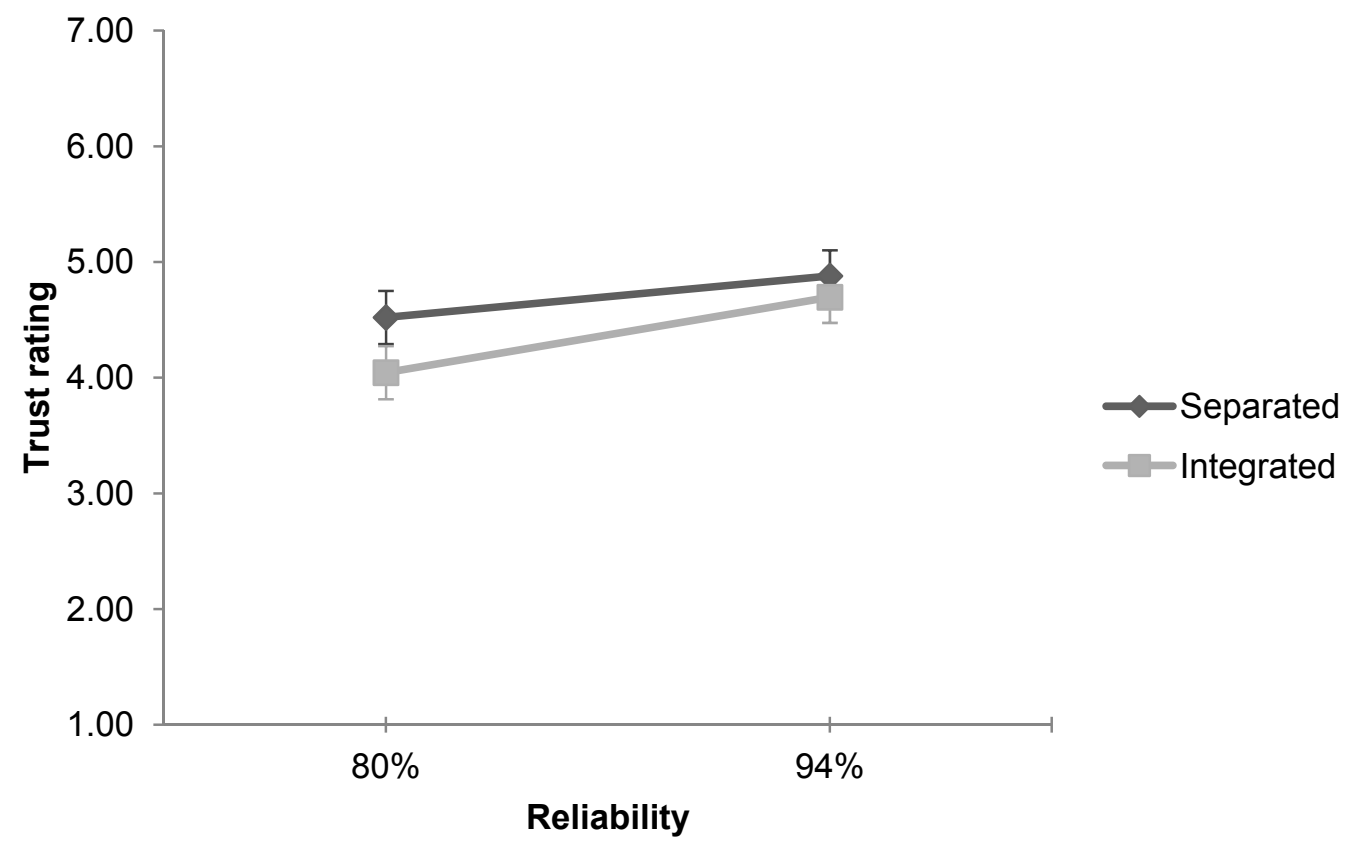

Figure 34. Effects of aid reliability and display on subjective trust raings 


\section{Discussion}

This section discusses the results of the display evaluation and places them in the context of using EID to foster appropriate trust and reliance in decision aids. Based on identified limitations of the conducted study, a subsequent study is proposed.

\subsection{Evaluation Results}

Despite the severe under-reliance indicated by the comparison of observed and effective $\ln \beta$ differences, participants exhibited some degree of reliance, as indicated by the $\ln \beta$ and $\mathrm{C}$ differences. Both measures showed that participants became more liberal in responding "signal" when given signal feedback for both changes in reliability (no-aid - 80\% and $80-94 \%$ ). Participants also became more conservative when given no-signal feedback. The misuse and disuse rates also indicated that participants relied on the aid at both reliability levels. However, the degrees to which participants relied upon the $80 \%$ and $94 \%$ reliable aids differed. Changes in $\mathrm{C}$ for both displays showed that participants changed their response biases more dramatically in the no-aid $-80 \%$ condition than the $80-94 \%$ condition. Given that participants under-relied upon the aid in every condition, reliance was more appropriate with the $80 \%$ reliable aid. This result can be confirmed graphically by comparing the effective and observed $\ln \beta$ differences, which indicate that the effective response bias shift was roughly equal for both the no-aid - $80 \%$ and $80-94 \%$ reliability changes (Figure 26 to Figure 29). A possible explanation for the observed under-reliance is that participants increasingly overestimated the probability of aid errors, resulting in sluggish $\beta$ values (Wickens \& Hollands, 2000).

The aid's display also appeared to affect reliance, but to a lesser degree. The $\mathrm{C}$ differences indicated that separated display users relied upon the aid more appropriately than integrated display users. Specifically, there was a greater difference in reliance between the displays for the $80-94 \%$ reliability difference: in Figure 30 and Figure 31, the slopes for the displays differed more in the $80-94 \%$ plot than in the no-aid $-80 \%$ plot. A similar difference appeared in the $\ln \beta$ plots (Figure 26 and Figure 27), where reliance is roughly equal in the no-aid - 80\% condition. Thus, both displays yielded similar reliance levels in the $80 \%$ reliable aid, but the separated display yielded appropriately higher reliance in the $94 \%$ reliable aid. 
Participants' detection performance reflected these reliance results. Participants' detection sensitivities, false alarm rates, and miss rates all improved as they increasingly relied upon the aid. However, the integrated display produced lower miss rates than the separated display when the aid was $80 \%$ reliable, but higher miss rates when it was $94 \%$ reliable. Based on the plot of miss rates (Figure 23), the difference in miss rates was greater in the $94 \%$ condition, which is consistent with the observation that separated display users demonstrated appropriately higher reliance upon the $94 \%$ reliable aid than integrated display users.

A possible explanation for this reliance difference is the way in which the reliability levels were instantiated. Reliability was increased by reducing the standard deviation of the alarm input values, rather than maintaining a constant deviation and selecting the required proportion of correct trials. To achieve $94 \%$ reliability, this manipulation resulted in many trials where the intermediate mass assignments were both very high or both very low. For such cases, the integrated display became highly salient as either the red or green rectangle would occupy a large majority of the grid. After being exposed to these salient trials, participants may have been less inclined to rely on subsequent results that were not of similar salience but still correct. Indicator salience has been found to affect signal detection performance: Montgomery and Sorkin (1996) found that coding the reliability of visual cues using luminance helped participants weigh the cues more appropriately. Meyer (2001) manipulated aid feedback salience by changing its proximity to the raw data and found that users had difficulty ignoring invalid warnings in cases of high proximity. These findings reflect a human tendency to minimize effort by relying on the most salient information (Lee, 2006).

Trust ratings were affected by only aid reliability, which was disclosed to participants before each experimental block. Such disclosure provided participants with performance-related information about automation capability (Lee \& See, 2004). Lee and Moray (1992) found that system performance and the occurrence of faults accounted for the majority of variance in participants' trust ratings for an automated system. Similarly, Wang et al. (2009) found that disclosing aid reliability significantly improved the appropriateness of reliance. Moreover, McGuirl and Sarter (2006) found that showing operators dynamic indications of a decision aid's confidence improved trust calibration and decision-making performance. With respect to faults, the independence between the alarm input values and the four cues often resulted in conflicts, which participants may have perceived as failure. These failures were particularly apparent in 
training, where aid reliability was not disclosed and feedback was provided following each trial. Consequently, participants may have reduced their trust and reliance upon the automation in subsequent trials. Muir and Moray (1996) found that negative experiences with automation affect trust more than positive experiences, while Dzindolet et al. (2001a) found that users of a reliable decision aid distrusted it after witnessing it commit errors.

\subsection{Ecological Interface Design for Decision Aids}

The more appropriate reliance and lower miss rates observed with the separated display in the highest aid reliability condition may suggest that the ecological display provided little to no benefit. The intermediate mass assignments shown in both displays may have provided sufficient process information. In Tsai et al.'s (2011) interactive visualization that improved Bayesian reasoning, a grid was used to represent the proportion of a population matching user-selected conditions (Figure 35). By updating the grid each time users added criteria, the visualization presented the intermediate results used to arrive at the final proportion without presenting the underlying relationship of Bayes' theorem. Jamieson, Wang, and Li (2006) also developed (but did not test) displays for Dempster's rule and only presented the intermediate mass assignments to supplement the joint assignments. Providing users with the intermediate mass assignments is consistent with Lee and See's (2004) suggestion that automation process can be communicated by revealing intermediate results.

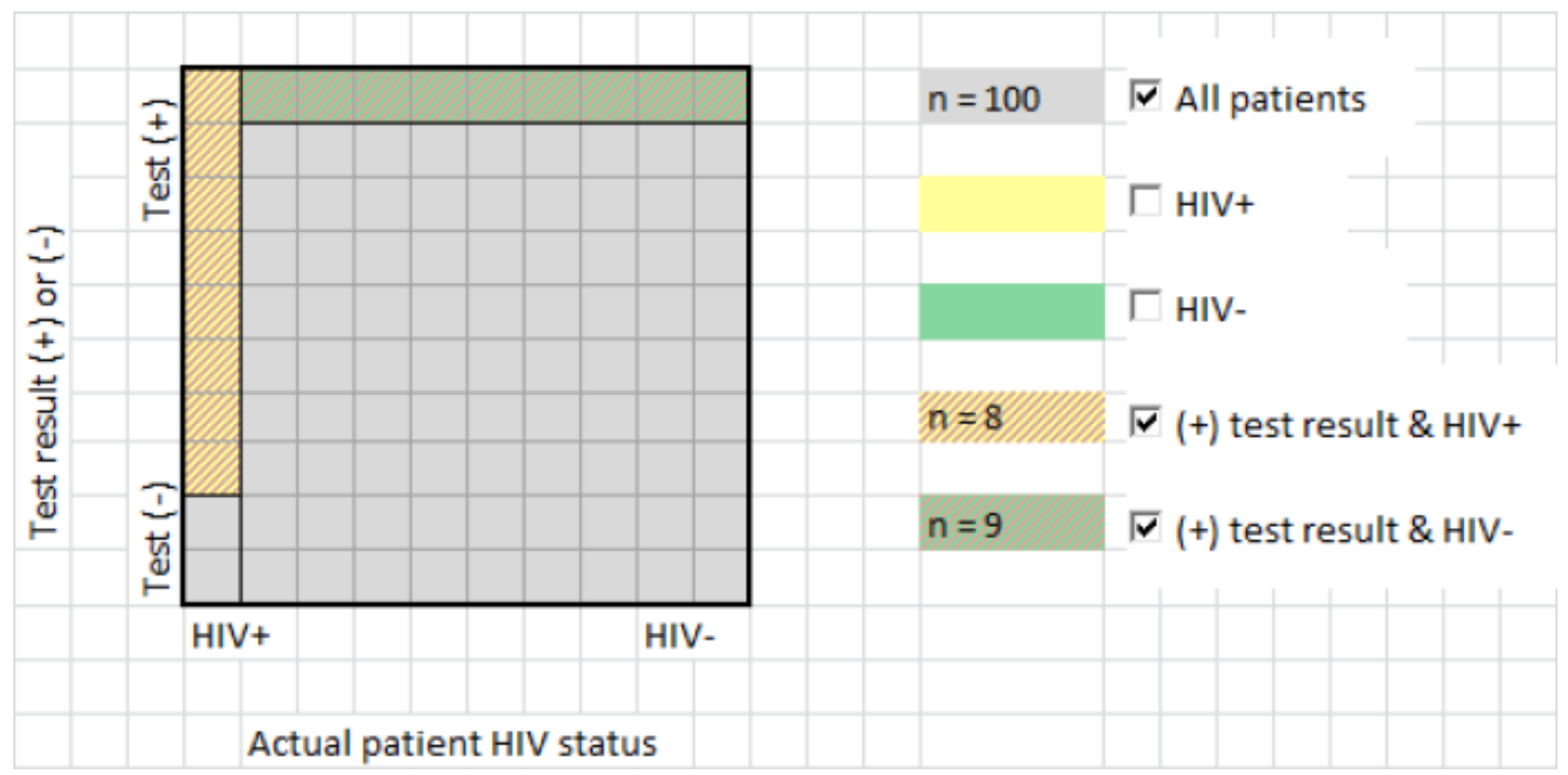

Figure 35. Interactive visualization for Bayes' law (from Tsai et al., 2011) 
Lee and See (2004) also suggest that automation algorithms and operations be simplified so that they are understandable, motivating the use of representation-aiding to visualize Dempster's rule of combination. In support of EID, Lee and See (2004) noted an apparent applicability of the AH and SRK frameworks to designing for appropriate trust. They suggest that the informational basis of trust in automation and the $\mathrm{AH}$ share common dimensions of attributional abstraction and system detail. Lee and See (2004) also consider the possible relationship between skill-, rule, and knowledge-based behaviour and the "analytic, analogical, and affective processes that govern trust" (p. 75). In practice, it was possible to apply the principles of EID to identify and display a higher level function of the IDWMS. However, despite the applicability of the approach, the resulting display yielded worse performance and less appropriate trust and reliance. These findings might suggest that the displays needed to support appropriate trust in automation differ from those needed to support the handling of routine and unanticipated events in complex work domains, which is the intent of EID (Vicente \& Rasmussen, 1992). Another possibility is that the findings were limited by the experimental methodology.

\subsection{Limitations}

The primary limitation of the conducted study was the independent generation of the alarm input values and cues. This approach resulted in frequent and unpredictable conflicts between the cues and aid results (confidence values and conclusions). Unpredictable faults have been found to significantly reduce trust (Muir \& Moray, 1996), and these conflicts were especially obvious when participants received feedback during training. This disconnect also resulted in no consistent indicator of context-specific aid reliability, which severely limits users' ability to judge automation capability (Lee \& See, 2004). Consequently, participants could not practice adaptive behaviour. The observed trust and reliance behaviour may thus be limited in meaning.

The lack of training for Dempster's rule may have limited the effectiveness of the integrated display. Studies on emergent features typically focus on their ability to aid mental integration of information by facilitating perceptual processing (Barnett \& Wickens, 1988; Wickens \& Andre, 1990). In order for the emergent features to be useful, participants in such studies are trained to manually perform the integration task. However, the integration task in the current study is performed by automation, and the current research interest lies in the potential for emergent features to help participants supervise and adapt to the automation, which is often more technically capable than the supervisor. 
The integrated display's appearance may have also limited the integrated display's effectiveness (Figure 36). The bottom half of the vertical meter and the left half of the horizontal meter are both green to represent the range of mass assignments for which the conclusion is "no-signal". At the same time, the red rectangle representing the intersection of the signal proposition resides in the lower-left corner of the grid. The high spatial proximity of these conflicting codes creates response competition and possibly hindered processing of the information (Wickens \& Hollands, 2000).

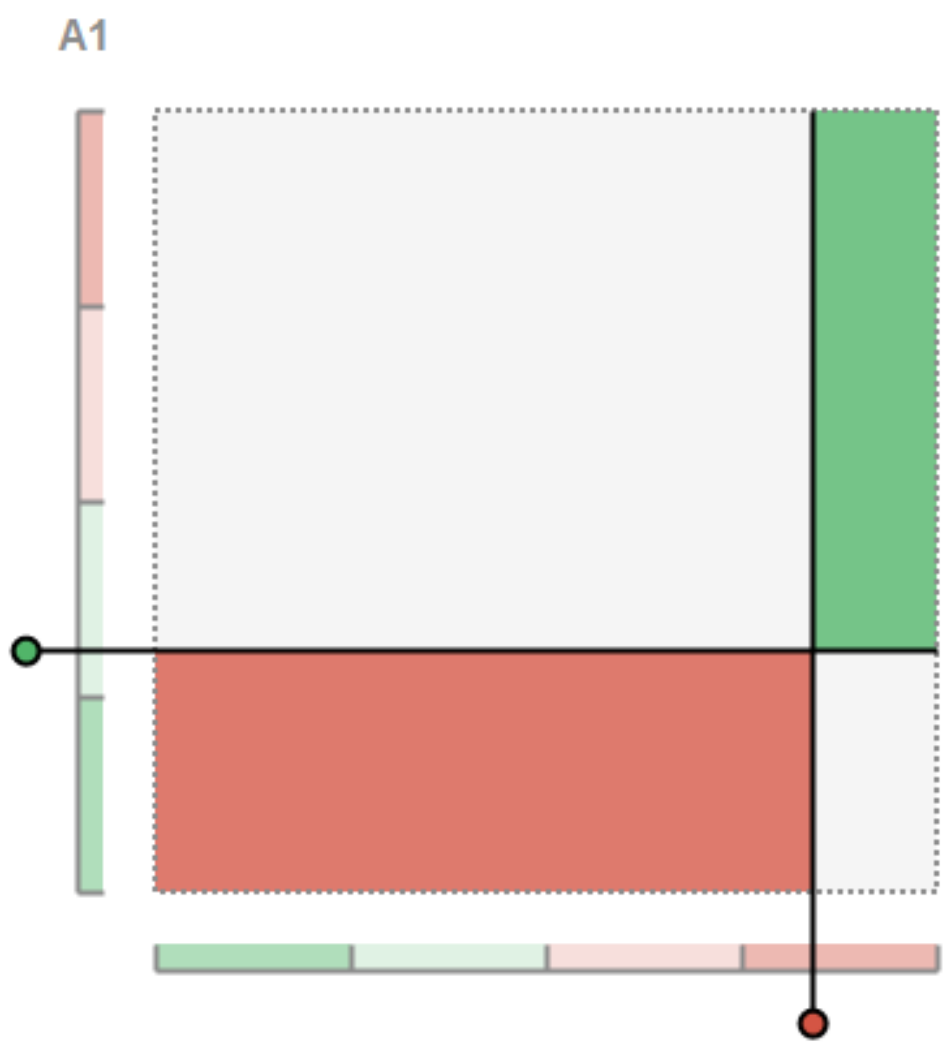

A2

Figure 36. Conflicting codes in the integrated display used for evaluation

With respect to the collected data, the high aid reliabilities used in the study prevented much of the data from being analyzed using the signal detection theory-based approach because many participants produced hit or false alarm rates equal to 1 or 0 for the rare trials in which the aid failed. While using high reliability rates is representative of real-world systems, it requires a large number of trials to study how participants behave when the automation fails. 


\subsection{Future Work}

As discussed in the Limitations section, the apparently random relationship between the cues and aid results did not give participants consistent indications of context-specific aid reliability to which reliance could be adapted. To obtain more meaningful observations of trust and reliance behaviour in a subsequent study, the aid's results should be consistently correlated with the visible cues such that participants can judge aid reliability and adapt appropriately to improve performance. As a possible experimental task, participants would be presented four cues that differ in their correlation with the true state of a system (e.g., signal or no-signal). Two "sensors" would generate different mass assignment functions by weighing the cues differently and participants would practice determining the state of the system using the sensor results and cues. The goal of this practice is for participants to develop an ability to judge the sensors' contextspecific reliabilities and adapt accordingly by adjusting the mass assignments. Dempster's rule of combination would be used as the decision aid's method for integrating both sensors' mass assignment functions.

With this revised task, representing Dempster's rule using an emergent feature might support participant adaptations to the automation. Specifically, the integrated display would show how the joint mass assignment function is affected by participants' adjustments of the sensor results. Thus, the participants' focus is not to verify the aid's fusion calculation, but to adjust its inputs based on perceived sensor faults. Sensor reliability is likely to be a greater concern for an implemented system given the consistency with which programmed calculations are executed.

Moreover, the display could allow users to input adjusted sensor mass assignments. In this case, both the integrated and separated displays would show the degree to which the joint mass assignments are affected, but the integrated display would also explain the joint result's response. In this way, the effect of presenting higher level functions via emergent features on trust, reliance, and performance can be more effectively studied. Interactivity may also be worth investigating in light of Tsai et al.'s (2001) successful Bayesian visualization. Finally, if this interaction is carried out via direct manipulation of the display, then the display could support skill-based behaviour (Vicente \& Rasmussen, 1992) and serve as further investigation of the applicability of EID's principles to supporting appropriate trust in decision aids. For example, participants might adjust the sensor mass assignments on the display by dragging the markers using a computer mouse. 
The hypothesis that the integrated display's salience reduced reliance should also be investigated. To do so, more data should be collected for highly salient trials, where the two intermediate mass assignment functions had high degrees of belief and agreement in the same proposition. The response biases for these trials should then be compared to the less salient trials.

In addition to a subsequent experiment, the applicability of EID to decision aid algorithms could be further investigated by designing graphic forms that map to the other IDWMS constraints. In particular, future work should display the relationship that integrates conventional parameter readings and threshold values to produce the conventional mass assignments. A more complex relationship that should also be investigated is the comparison of sample and reference spectra to generate the spectroscopic mass assignments. Before such design work can be started, a thorough understanding of these relationships must be obtained. 


\section{Conclusion}

This thesis aimed to improve joint human-automation decision-making performance by fostering appropriate trust and reliance via display design. In the case of the IDWMS, operators are described as supervisors who must monitor the automation and adapt their reliance to its contextspecific capabilities. To support such human-automation interaction, the current work investigated EID's applicability to satisfying Lee and See's (2004) design guidelines for displays that support appropriate trust. EID informed both the content and form of the resulting display.

The ADS was used to model the IDWMS and the resulting levels of abstraction provided information that described automation capability. The functional purpose level described the intended use of the automation level, while the abstract, generalized, and physical function levels gave conceptually different descriptions of automation process. The Dempster-Shafer theory was identified as a higher order system constraint to be made directly perceptible to operators. An emergent feature mapping to Dempster's rule of combination was designed to help operators supervise the IDWMS sensor fusion function and adapt to its context-specific reliability.

The integrated display was compared to a separated display in a signal detection theory-based study that allowed participants' reliance on a decision aid to be measured using response bias. Results indicated that the separated display yielded more appropriate reliance, better performance, and equal trust when participants used a 94\% reliable decision aid. Performance, reliance, and trust were roughly equal between both displays with an $80 \%$ reliable aid. These results suggest that the additional process information in the integrated display was not beneficial and question the applicability of EID to supporting appropriate trust. However, the experimental task did not give participants consistent indications of the decision aid's context-specific reliability and may have limited the validity of the observed trust and reliance behaviours. A subsequent study is proposed to more effectively investigate the applicability of EID's principles to supporting appropriate trust and reliance on automation. 


\section{References}

Bainbridge, L. (1983). Ironies of automation. Automatica, 19, 775-779.

Barnett, B. J., \& Wickens, C. D. (1988). Display proximity in multicue information integration: The benefits of boxes. Human Factors, 30(1), 15-24.

Bennett, K. B., Flach, J. M. (1992). Graphica displays: Implications for divided attention, focused attention, and problem solving. Human Factors, 34(5), 513-533.

Bennett, K. B., Nagy, A. L., \& Flach, J. M. (2006). Visual displays. In G. Salvendy (Ed.), Handbook of Human Factors and Ergonomics (3rd ed., pp. 1191-1221). Hoboken, NJ: John Wiley \& Sons.

Burns, C. M. (2000). Putting it all together: Improving display integration in ecological displays. Human Factors, 42(2), 226-241.

Burns, C. M., Bisantz, A. M., \& Roth, E. M. (2004). Lessons from a comparison of work domain models: Representational choices and their implications. Human Factors, 46(4), 711-727.

Burns, C. M., \& Hajdukiewicz, J. R. (2004). Ecological interface design. Boca Raton, FL: CRC Press.

Burns, K. (2004). Bayesian boxes: A colored calculator for picturing posteriors. In Proceedings of the 3rd International Conference, Diagrams 2004 (pp. 382-384). Cambridge, UK: Springer.

Carswell, M. C., \& Wickens, C. D. (1996). Mixing and matching lower-level codes for object displays: Evidence for two sources of proximity compatibility. Human Factors, 38(1), 122.

Clark, R. M., Hakim, S., \& Ostfield, A. (2011). Handbook of Water and Wastewater Systems Protection. New York, NY: Springer.

Cleveland, W. S., \& McGill, R. (1984). Graphical perception: Theory, experimentation, and application to the development of graphical methods. Journal of the American Statistical Association, 79(387), 531-554.

Cooke, N. J. (1994). Varieties of knowledge elicitation techniques. International Journal of Human-Computer Studies, 41, 801-849.

Degani, A., \& Heymann, M. (2002). Formal verification of human-automation interaction. Human Factors, 44(1), 28-43. 
Duez, P. P., Zuliani, M. J., \& Jamieson, G. A. (2006). Trust by design: Information requirements for appropriate trust in automation. In Proceedings of the 2006 Conference of the Center for Advanced Studies on Collaborative Research (pp. 108-118). New York, NY: ACM.

Dzindolet, M. T., Pierce, L. G., Beck, H. P., Dawe, L. A., \& Anderson, B. W. (2001a). Predicting misuse and disuse of combat identification systems. Military Psychology, 13(3), 147-164.

Dzindolet, M. T., Pierce, L. G., Pomranky, R., Peterson, S., \& Beck, H. (2001b). Automation reliance on a combat identification system. In Proceedings of the 45th Annual Meeting of the Human Factors and Ergonomics Society (pp. 532-536). Minneapolis/St. Paul, MN: Human Factors and Ergonomics Society.

Green, D. M., \& Swets, J. A. (1966). Signal detection theory and psychophysics. New York: Wiley.

Guerlain, S., Jamieson, G. A., Bullemer, P., \& Blair, R. (2002). The MPC elucidator: A case study in the design for human-automation interaction. IEEE Transactions on Systems, Man, and Cybernetics - Part A: Systems and Humans, 32(1), 25-39.

Heymann, M., \& Degani, A. (2007). Formal analysis and automatic generation of user interfaces: Approach, methodology, and an algorithm. Human Factors, 49(2), 331-330.

Ho, D. \& Burns, C. M. (2003). Ecological interface design in aviation domains: Work domain analysis of automated collision detection and avoidance. In Proceedings of the Human Factors and Ergonomics Society 47th Annual Meeting (pp. 119-123), Santa Monica, CA: Human Factors and Ergonomics Society.

Hoffman, R. R., Shadbolt, N. R., Burton, A. M., \& Klein, G. (1995). Eliciting knowledge from experts: A methodological analysis. Organizational behaviour and human decision processes, 62(2), 129-158.

Howell, D. C. (2010). Statistical methods for psychology (7th ed.). Belmont, CA: Cengage Wadsworth.

Jamieson, G. A., \& Vicente, K. J. (2005). Designing effective human-automation-plant interfaces: A control theoretic perspective. Human Factors, 47(1), 12-34.

Jamieson, G. A., Wang, L., \& Li, J. (2006). Developing human-machine interfaces to support monitoring of UAV automation (CEL 06-02). Toronto, Ontario: Cognitive Engineering Laboratory.

Jian, J., Bisantz, A. M., \& Drury, C. G. (2000). Foundations for an empirically determined scale of trust in automated systems. International Journal of Cognitive Ergonomics, 4, 53-71.

Lee, J. D., (2006). Human factors and ergonomics in automation design. In G. Salvendy (Ed.), Handbook of human factors and ergonomics (3rd ed., pp. 1570-1596). Hoboken, NJ: John Wiley \& Sons. 
Lee, J. D., \& Moray, N. (1992). Trust, control strategies and allocation of function in humanmachine systems. Ergonomics, 35, 1243-1270.

Lee, J. D., \& See, K. A. (2004). Trust in automation: Designing for appropriate reliance. Human Factors, 46(1), 50-80.

Lind, M. (1999). Plant modelling for human supervisory control. Transactions of the Institute of Measurement and Control, 21(4-5), 171-180.

Lind, M. (2003). Making sense of the abstraction hierarchy in the power plant domain. Cognition, Technology \& Work, 5(2), 67-81.

Macmillan, N. A., \& Creelman, C. D. (1991). Detection theory: A user's guide. New York, NY: Cambridge University Press.

Maltz, M., \& Meyer, J. (2001). Use of warnings in an attentionally demanding detection task. Human Factors, 43, 217-226.

Maltz, M., \& Shinar, D. (2003). New alternative methods of analyzing human behavior in cued target acquisition. Human Factors, 45(2), 281-296.

Mazaeva, N. \& Bisantz. A. M. (2007). On the representation of automation using a work domain analysis. Theological Issues in Ergonomic Sciences, 8(6), 509-530.

McGuirl, J. M., \& Sarter, N. B. (2006). Supporting trust calibration and the effective use of decision aids by presenting dynamic system confidence information. Human Factors, 48(4), 656-665.

Mesarovic, M. D., Macko, D., \& Takahara, Y. (1970). Theory of hierarchical, multilevel systems. New York: Academic Press.

Meyer, J. (2001). Effects of warning validity and proximity on responses to warnings. Human Factors, 43, 563-572.

Meyer, J., \& Bitan, Y. (2002). Why better operators receive worse warnings. Human Factors, 44(3), 343-353.

Miller, A., \& Sanderson, P. M. (2000). Modelling "deranged” physiological systems for ICU information system design. In Proceedings of the Joint 14th Triennial Congress of the International Ergonomics Association/44th Annual Meeting of the Human Factors and Ergonomics Society (pp. 245-248). Santa Monica, CA: Human Factors and Ergonomics Society.

Montgomery, D. A., \& Sorkin, R. D. (1996). Observer sensitivity to element reliability in a multielement visual display. Human Factors, 38, 484-494. 
Mosier, K. L., \& Skitka, L. J. (1996). Human decision makers and automated decision aids: Made for each other? In R. Parasuraman \& M. Mouloua (Eds.), Automation and human performance: Theories and applications (pp. 201-220). Mahwah, NJ: Lawrence Erlbaum Associates.

Muir, B. M., \& Moray, N. (1996). Trust in automation. Part II. Experimental studies of trust and human intervention in a process control simulation. Ergonomics, 39, 429-360.

Murrell, G. (1977). Combination of evidence in a probabilistic visual search and detection task. Organizational Behaviour and Human Performance, 18, 3-18.

Naikar. N., Hopcroft, R., \& Moylan, A. (2005) Work domain analysis: Theoretical concepts and methodology (DSTO-TR-1665). Fishermans Bend, Australia: Defence Science and Technology Organisation.

Neyedli, H. F., Hollands, J. G., \& Jamieson, G. A. (2011). Beyond identity: Incorporating system reliability information into an automated combat identification system. Human Factors, 53(4), 338-355.

Parasuraman, R., \& Riley, V. A. (1997). Humans and automation: Use, misuse, disuse, abuse. Human Factors, 39(2), 230-253.

Parasuraman, R., Sheridan, T. B., \& Wickens, C. D. (2000). A model of types and levels of human interaction with automation. IEEE Transactions on Systems, Man, and Cybernetics, 30, 286-297.

Pawlak, W. S., \& Vicente, K. J. (1996). Inducing effective operator control through ecological interface design. International Journal of Human-Computer Studies, 44, 653-688.

Pomerantz, J. R., \& Pristach, E. A. (1989). Emergent features, attention, and perceptual glue in visual form perception. Journal of Experimental Psychology: Human Perception and Performance, 15, 635-649.

Rasmussen, J. (1983). Skills, rules and knowledge: Signals, signs, and symbols and other distinctions in human performance models. IEEE Transactions on Systems, Man, and Cybernetics, SMC-13(3), 257-267.

Reising, D. V. C. (2000). The abstraction hierarchy and its extension beyond process control. Human Factors, 44(1), 194-197.

Reising, D. V. C., \& Sanderson, P. M. (2002). Ecological interface design for pasteurizer II: A process description of semantic mapping. Human Factors, 44(2), 222-247.

Robinson, D. E., \& Sorkin, R. D. (1985). A contingent criterion model of computer assisted detection. In R. Eberts \& C. G. Eberts (Eds.), Trends in ergonomics/human factors (Vol. 2, pp. 75-83). Amsterdam: North-Holland. 
Safar, J. A., \& Turner, C. W. (2005). Validation of a two factor structure for system trust. In Proceedings of the Human Factors and Ergonomics Society 49th Annual Meeting (pp. 497-501), Orlando, FL: Human Factors and Ergonomics Society.

See, J. E., Warm, J. S, Dember, W. N, \& Howe, S. R. (1997). Vigilance and signal detection theory: An empirical evaluation of five measures of response bias. Human Factors, 39(1), 14-29.

Sentz, K. (2002). Combination of evidence in Dempster-Shafer theory (SAND 2002-0835). Sandia National Laboratories.

Shafer, G. (1976). A mathematical theory of evidence. Princeton, NJ: Princeton University Press.

Sheridan, T. B. (2006). Supervisory control. In G. Salvendy (Ed.), Handbook of Human Factors and Ergonomics (pp. 1025-1052). New York: Wiley.

Sheridan, T. B., \& Verplank, W. L. (1978). Human and computer control of undersea teleoperators. Cambridge, MA: MIT.

Sorkin, R. D., Kantowitz, B. H., \& Kantowitz, S. C. (1988). Likelihood alarm displays. Human Factors, 30(4), 445-459.

Sorkin, R. D., \& Woods, D. D. (1985). Systems with human monitors: A signal detection analysis. Human-Computer Interaction, 1, 49-75.

Spain, R. D., Bustamante, E. A., \& Bliss, J. (2008). Towards an empirically developed scale for system trust: Take two. In Proceedings of the Human Factors and Ergonomics Society 52th Annual Meeting (pp. 1335-1339), New York, NY: Human Factors and Ergonomics Society.

Tsai, J., Miller, S., \& Kirlik, A. (2011). Interactive visualizations to improve Bayesian reasoning. In Proceedings of the Human Factors and Ergonomics Society 55th Annual Meeting (pp. 385-389), Las Vegas, NV: Human Factors and Ergonomics Society.

Tversky, A., \& Kahneman, D. (1974). Judgment under uncertainty: Heuristics and biases. Science, 185, 1124-1131.

Vicente, K. J. (1999). Cognitive Work Analysis: Towards safe, productive, and healthy computer-based work. Mahwah, NJ: Lawrence Erlbaum Associates.

Vicente, K. J., \& Rasmussen, J. (1992). Ecological interface design: Theoretical foundations. IEEE Transactions on Systems, Man and Cybernetics, 22, 589-606.

Wang, L., Jamieson, G. A., \& Hollands, J. G. (2009). Trust and reliance on an automated combat identification system. Human Factors, 51(3), 281-291. 
Wickens, C. D., \& Andre, A. D. (1990). Proximity compatibility and information display: Effects of color, space, and objectness of information integration. Human Factors, 32(1), 61-77.

Wickens, C. D., \& Carswell, C. M. (1995). The proximity compatibility principle: Its psychological foundation and relevance to display design. Human Factors, 37(3), 473494.

Wickens, C. D., \& Hollands, J. G. (1999). Engineering psychology and human performance (3rd ed.). Upper Saddle River, NJ: Prentice Hall. 


\section{Appendix A: IDWMS Display}

The entire designed IDWMS display is presented in the following images. The first image is an overview screen of all the sensor sites installed in a distribution network. The contour plot discussed in the body of this thesis presents operators with the current spectroscopic, conventional aggregate and final mass assignments. In the second image, this contour plot is replaced with three trend charts to indicate the historical values of each mass assignment. The third image presents the detailed information for each sensor site, including the Dempster's rule of combination grid display, compared spectra, and individual conventional parameter readings. 


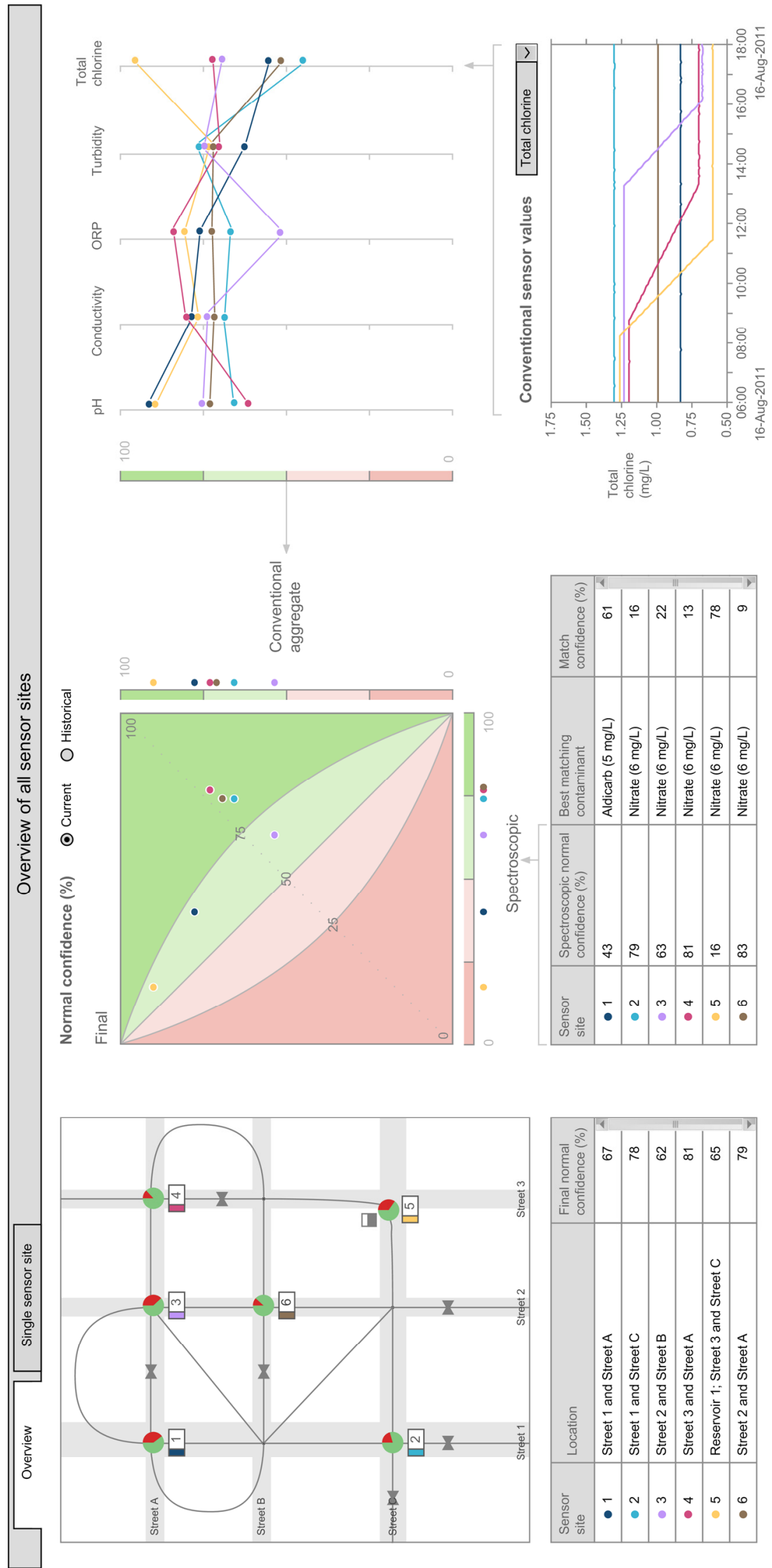




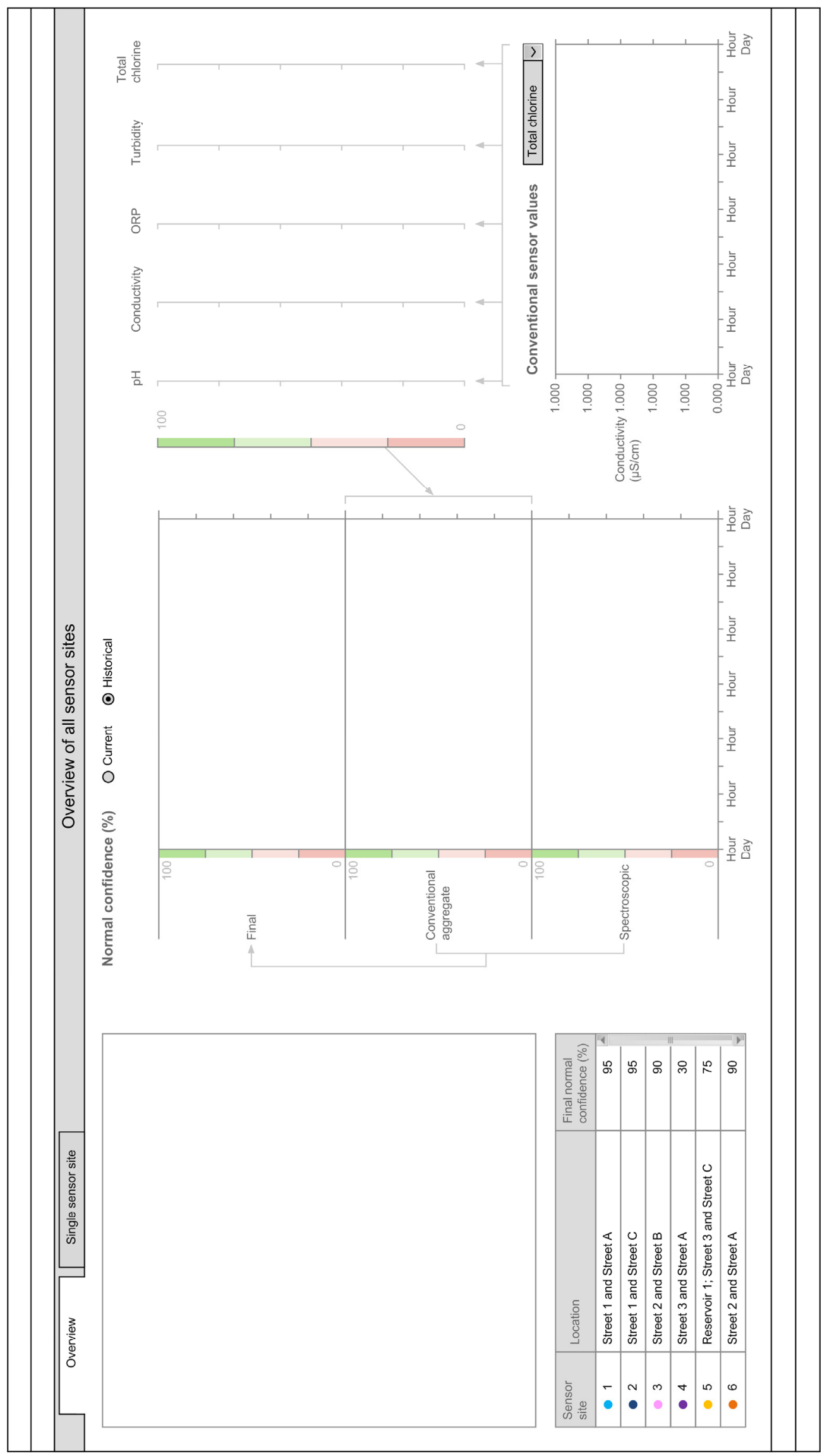




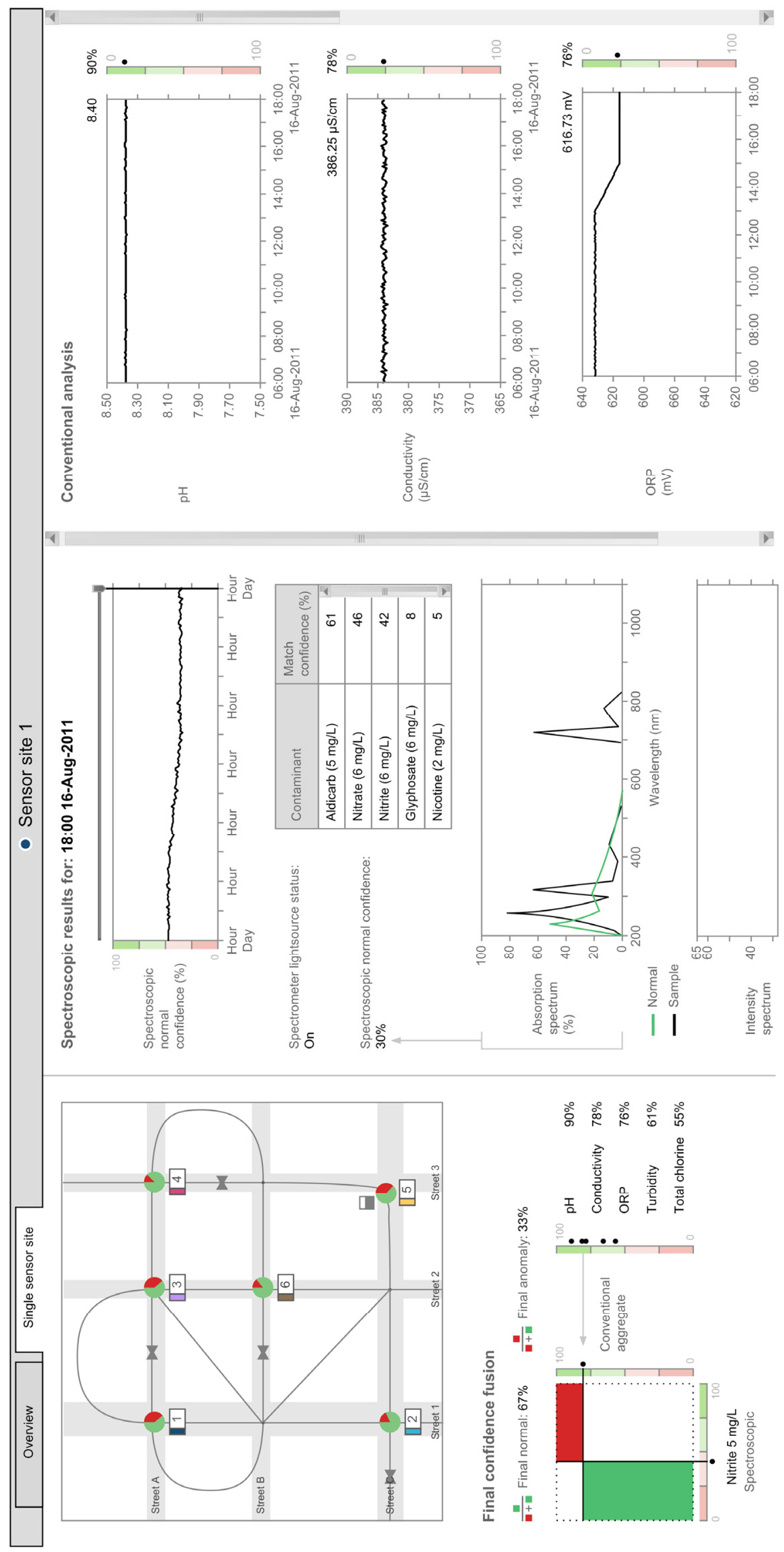




\section{Appendix B: Informed Consent Form}

\section{Effects of display type on performance, trust, and reliance in a signal detection task}

Principal investigator: Kevin Kan (416-978-0881; kan@mie.utoronto.ca)

Faculty supervisor: Prof. Greg A. Jamieson (416-946-8504; jamieson@mie.utoronto.ca)

Department of Mechanical and Industrial Engineering

University of Toronto

The purpose of this study is to evaluate the effects of a decision aid user interface on users' performance, trust, and reliance when performing a signal detection task. The interface to be tested has been designed to provide information about the aid's algorithm and intermediate results, so that users may have a better understanding the aid. The results of this study may be used to inform interface design for an industry product.

You are invited to participate in this study because you are an engineering student at the University of Toronto. There will be approximately 36 participants involved.

\section{Procedure}

You will be asked to perform the following tasks if you choose to participate in this experiment. The experiment is expected to last 2 hours.

1. You will complete a demographic questionnaire about year and discipline of education, as well as previous exposure to Dempster Shafer Theory.

2. You will be given training and practice for performing the experimental task and using the user interface.

3. You will perform a signal detection task manually and using the user interface. Series of 4 numbers will be presented to you, and you will determine whether the numbers indicate an event or not.

4. You will complete a questionnaire assessing your trust in the decision aid.

\section{Risks}

There are no known or anticipated risks for participants in this study. There are no physiologically demanding tasks in this study, and no psychological manipulation or deception. Your performance on the tasks will not be used for any other purpose than to study human interaction with the decision aid and its user interface.

\section{Benefits}

You may benefit from gaining exposure to Dempster Shafer Theory.

\section{Compensation}

You will receive $\$ 10$ per hour (rounded to the nearest 30 minutes), to a maximum of $\$ 20$ for completing this study. The total compensation amount will be paid in one full payment at the end 
of the study. If you wish to withdraw from the study before completion, you will be compensated for the amount of time that you spent participating (rounded to the nearest 30 minutes).

\section{Confidentiality}

Your privacy and identity will be carefully protected in this study. Your data will be kept securely, and will only be accessed by the investigator and future researchers within the investigator's lab. In any publication, information will be provided in such a way that you cannot be identified. Your participation and identity will not be disclosed to others. You may request that your data be withdrawn and destroyed after your participation and debriefing, provided that it has not yet been analyzed and reported.

\section{Participation}

Your participation in this study is completely voluntary. You may decline to participate or decline to answer any question without any negative consequences. In addition, you may withdraw from the study at any time without any penalty, and request your data be destroyed.

\section{Contacting the investigators}

M.A.Sc. candidate Kevin Kan is undertaking this study in partial fulfillment of his degree requirements. If you have any additional questions later about this study or would like a summary of the research results, Kevin (kan@mie.utoronto.ca; 416-978-0881) will assist you. For information about participants' rights in scientific study, you can contact the University of Toronto's Ethics Review Office (ethics.review@utoronto.ca; 416-946-3273).

You will be given a completed copy of this form to keep.

\section{Participation Consent}

I have read this Informed Consent Form. I have had the opportunity to ask any questions that I had regarding the study, and I have received satisfactory answers to those questions. By my signature I affirm that I agree to take part in this study with the understanding that I can withdraw at any point. I have received a copy of this Informed Consent Form.

Participant signature

Participant name (please print)

Date
Investigator signature

Investigator name (please print)

Date 


\section{Appendix C: Demographic Questionnaire}

1. What degree and program are you currently enrolled in? (e.g., BASc in mechanical engineering)

2. What is your current year of study for your current degree? (e.g, a third year student)

3. If you already hold any post-secondary degrees, please list the degree and program. (e.g., BASc in electrical engineering)

4. If you have previous experience with Dempster Shafer Theory, please answer the following questions:

a. Briefly describe your experience with Dempster Shafer Theory. (e.g., learning and using it in a school course)

b. When did you last learn or use Dempster Shafer Theory? (month and year) 


\section{Appendix D: Training Material}

The training material given to evaluation participants to read is presented in the following pages.

Participants received the version of section 3.2 that corresponded to their display (the copies

given to participants were not titled with the display condition as they are here). 


\subsection{Your task}

You are monitoring a value $\mathrm{X}$ (Figure 1). There are two cases: either $\mathrm{X}$ is high $(\mathrm{X}=5)$ or $\mathrm{X}$ is normal $(\mathrm{X}=4)$.However, $\mathrm{X}$ is in a "black box" that prevents you from knowing the true value of $\mathrm{X}$.

Instead, you have 4 sensors that give estimates of X. For example, you may be shown:

Using these 4 values, your task is to determine if $X$ is high $(X=5)$, or if $X$ is normal $(X=4)$ :

- If $X$ is high, then the four estimates are likely to be clustered around 5 .

- If $X$ is normal, then the estimates are likely to be clustered around 4.

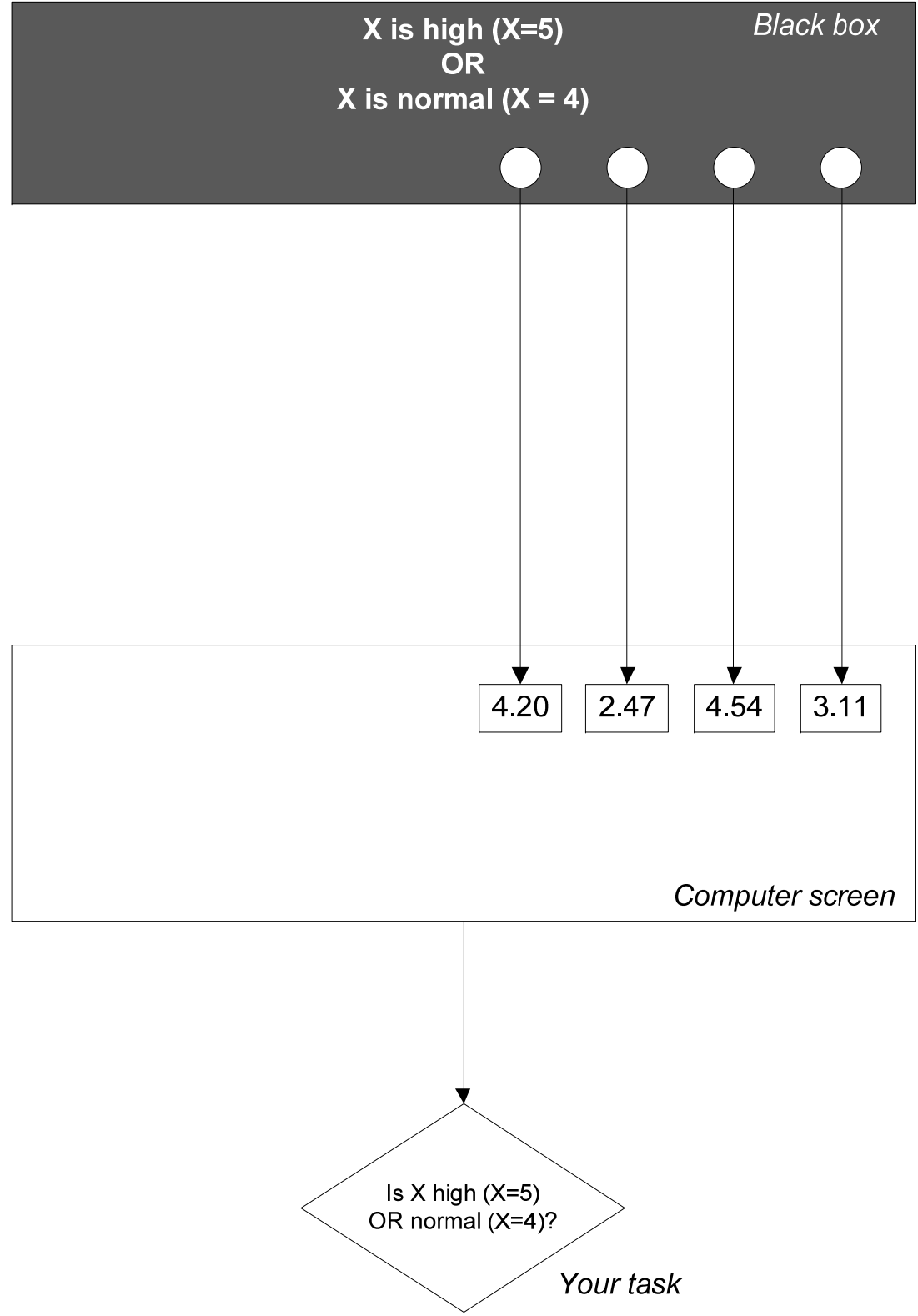

Figure 1. A flow chart of the task you will perform in each trial with the help of the decision aid. 
For example:

- The numbers $6.24,7.03,5.57,4.04$ likely indicate that $\mathrm{X}$ is high.

- The numbers $2.98,4.13,2.62,5.14$ likely indicate that $\mathrm{X}$ is normal.

- The numbers $4.71,3.04,5.48,6.07$ are more ambiguous than the previous two examples. In such cases, you must use your best judgment to decide whether $\mathrm{X}$ is normal or high.

\subsection{Manual practice trials: instructions}

Next, you will perform a block of 40 manual practice trials.

- In this block, half the trials contain a high $\mathrm{X}$ value, while the other half contains a normal X value.

- Weigh right and wrong answers equally - correctly identifying the state of $\mathrm{X}$ is as important as avoiding a mistake.

- You will be told if you were right or wrong after each trial. In some trials, it will be very difficult to determine if X is high or normal, so don't feel frustrated if you make mistakes. 


\subsection{The decision aid}

To help you decide on the state of X, a decision aid automatically analyzes the "black box" to estimate if X is high or normal (left side of Figure 2). The decision aid's results are shown to you in the user interface (Figure 3).

\subsection{Analysis 1 and Analysis 2}

The decision aid performs two analyses, A1 and A2. Both analyses use their own estimates of $X$, and not the four that are given to you. Each analysis produces two confidence values:

- The confidence that $\mathrm{X}$ is high: $\mathrm{A} 1_{\text {high }}$ and $\mathrm{A} 2_{\text {high }}$

- The confidence that $\mathrm{X}$ is normal: $\mathrm{A} 1_{\text {normal }}$ and $\mathrm{A} 2_{\text {normal }}$

- Both $\mathrm{A} 1_{\text {high }}+\mathrm{A} 1_{\text {normal }}$ must sum to $100 \%$

- If $\mathrm{A} 1_{\text {high }}>\mathrm{A} 1_{\text {normal, }}$, then $\mathrm{A} 1$ 's conclusion is: $\mathrm{X}$ is high

- If $\mathrm{A} 1_{\text {high }}<\mathrm{A} 1_{\text {normal, }}$, then $\mathrm{A} 1$ 's conclusion is: $\mathrm{X}$ is normal

- The same rules apply to $\mathrm{A} 2_{\text {high }}$ and $\mathrm{A} 2_{\text {normal }}$

For example, $\mathrm{A} 1$ might be $40 \%$ confident that $\mathrm{X}$ is high $\left(\mathrm{A} 1_{\text {high }}=0.40, \mathrm{~A} 1_{\text {normal }}\right.$ $=0.60)$. At the same time, $\mathrm{A} 2$ might be $67 \%$ confident that $\mathrm{X}$ is high $\left(\mathrm{A} 2_{\text {high }}=\right.$ $0.67, \mathrm{~A} 2_{\text {normal }}=0.33$ ).
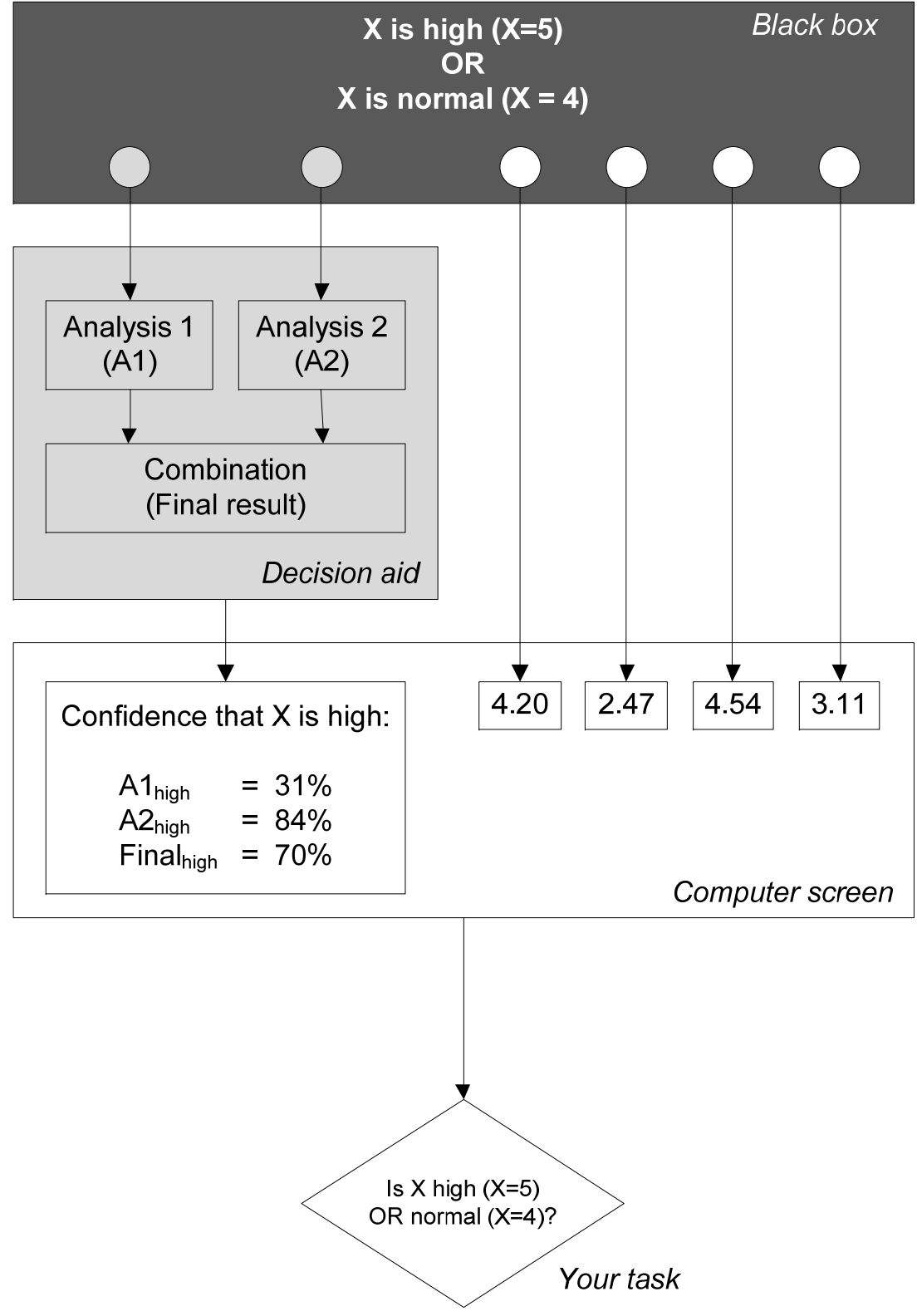

Figure 2. A flow chart of the task you will perform in each trial with the help of the decision aid. 
On the user interface (Figure 3), meters represent the confidence that $\mathrm{X}$ is high (as $\mathrm{A} 1_{\text {high }}$ and $\mathrm{A} 2_{\text {high }}$ increase, their markers move towards the red region). All the meters behave the same way, so A1 will be used as an example:

- At the meter's halfway point, $\mathrm{A} 1_{\text {high }}=\mathrm{A} 1_{\text {normal }}=0.50$.

- If $\mathrm{A} 1_{\text {high }}>\mathrm{A} 1_{\text {normal, }}$, then $\mathrm{A} 1$ concludes that $\mathrm{X}$ is high. On the meter, the marker will be above the halfway point (in the red region), and the marker will be red. For example, in Figure 3, $\mathrm{A} 2_{\text {high }}=0.84$ and $\mathrm{A} 2_{\text {normal }}=0.16$.

- If $\mathrm{A} 1_{\text {high }}<\mathrm{A} 1_{\text {normal, }}$, then $\mathrm{A} 1$ concludes that $\mathrm{X}$ is normal; the marker will be green and below the halfway point on the meter (in the green region). For example, in Figure 3, $\mathrm{A} 1_{\text {high }}=0.31$ and $\mathrm{A} 1_{\text {normal }}=0.69$.

\section{Reliability}

Both A1 and A2 are equally effective at determining if X is normal or high. However, neither A1 nor A2 is $100 \%$ reliable. Reliability refers to the percentage of trials in which an analysis' conclusion is correct. For example, if A1 is $75 \%$ reliable, then:

- For 100 trials where $\mathrm{X}$ is actually normal, $\mathrm{A} 1$ will correctly conclude that $\mathrm{X}$ is normal in only 75 trials (and incorrectly conclude that $\mathrm{X}$ is high in the other 25 trials).

- Likewise, for 100 trials where $\mathrm{X}$ is actually high, $\mathrm{A} 1$ will correctly conclude that $\mathrm{X}$ is high in only 75 trials (and incorrectly conclude that $\mathrm{X}$ is normal in the other 25 trials).

Since neither is $100 \%$ reliable, the $\mathrm{A} 1$ and $\mathrm{A} 2$ conclusions can sometimes disagree. For example, $\mathrm{A} 1_{\text {high }}=0.80$ while $\mathrm{A} 2_{\text {high }}=0.40$ (therefore, one conclusion must be incorrect). 


\subsection{The final result (separated display)}

Lastly, the decision aid combines the A1 and A2 confidence values to calculate the final confidence that $\mathrm{X}$ is high, and the final confidence that $\mathrm{X}$ is normal (Final ${ }_{\text {high }}$ and Final $1_{\text {normal }}$ ). On the user interface, the final conclusion and confidence values are shown in the same way as A1 and A2 (Figure 3 ).

The final confidence calculation ignores the amount of disagreement between A1 and A2. For example, all of the following combinations of A1 and A2 confidences produce the same final confidences $\left(\right.$ Final $_{\text {normal }}=0.90$, Final $\left.{ }_{\text {high }}=0.10\right)$ :

- $\mathrm{A} 1_{\text {normal }}=0.80$ (and $\left.\mathrm{A} 1_{\text {high }}=0.20\right)$ and

$\mathrm{A} 2_{\text {normal }}=0.70$ (and $\mathrm{A} 2_{\text {high }}=0.30$ )

- $\mathrm{A} 1_{\text {normal }}=0.93$ and $\mathrm{A} 2_{\text {normal }}=0.40$

- $\mathrm{A} 1_{\text {normal }}=0.60$ and $\mathrm{A} 2_{\text {normal }}=0.85$

The final conclusion is more reliable than the individual A1 and A2 conclusions. However, it is still not $100 \%$ reliable - when determining whether $\mathrm{X}$ is high or normal, you should consider each set of confidence values, as well as the four estimates of $\mathrm{X}$. 


\subsection{The final result (integrated display)}

Lastly, the decision aid combines the $\mathrm{A} 1$ and $\mathrm{A} 2$ confidence values to calculate the final confidence that $\mathrm{X}$ is high, and the final confidence that $\mathrm{X}$ is normal (Final ${ }_{\text {high }}$ and Final $1_{\text {normal }}$ ). On the user interface, the final conclusion and confidence values are shown in the same way as A1 and A2 (Figure 3 ).

In addition to the meters, the user interface will show a grid that is created by extending the markers on the A1 and A2 meters (Figure 3 ). This grid shows how the A1 and A2 confidence values are combined to calculate the final confidence values. The red area represents the amount that A1 and A2 agree that $\mathrm{X}$ is high. The green area represents the amount that $\mathrm{A} 1$ and $\mathrm{A} 2$ agree that $\mathrm{X}$ is normal. Final ${ }_{\text {high }}$ is represented as the proportion of red area to coloured (red or green) area. For example, if the green area shrinks and the red area grows, then Final ${ }_{\text {high }}$ will increase (to a maximum of $100 \%$ ).

Note that the final result calculation ignores the amount of disagreement between A1 and A2, since it does not consider how much uncoloured area exists. For example, all of the following combinations of A1 and A 2 confidences produce the same final confidences $\left(\right.$ Final $_{\text {normal }}=0.90$, Final $\left.l_{\text {high }}=0.10\right)$ :

- $\mathrm{A} 1_{\text {normal }}=0.80\left(\right.$ and $\left.\mathrm{A} 1_{\text {high }}=0.20\right)$ and

$\mathrm{A} 2_{\text {normal }}=0.70$ (and $\mathrm{A} 2_{\text {high }}=0.30$ )

- $\mathrm{A} 1_{\text {normal }}=0.93$ and $\mathrm{A} 2_{\text {normal }}=0.40$

- $\mathrm{A} 1_{\text {normal }}=0.60$ and $\mathrm{A} 2_{\text {normal }}=0.85$

The final conclusion is more reliable than the individual A1 and A2 conclusions. However, it is still not $100 \%$ reliable - when determining whether X is high or normal, you should consider each set of confidence values, as well as the four estimates of X. 


\subsection{Aided practice trials: instructions}

Next, you will perform a block of 40 practice trials with the help of the decision aid:

- The final, A1, and A2 conclusions will not be $100 \%$ reliable.

- The final conclusion is more reliable than the individual A1 and A2 conclusions.

- The A1 and A2 conclusions are equally reliable.

Similar to the manual practice trials:

- In this block, half the trials contain a high $\mathrm{X}$ value, while the other half contains a normal $\mathrm{X}$ value.

- Weigh right and wrong answers equally - correctly identifying the state of $\mathrm{X}$ is as important as avoiding a mistake.

- You will be told if you were right or wrong after each trial. In some trials, it will be very difficult to determine if $\mathrm{X}$ is high or normal, so don't feel frustrated if you make mistakes. 


\subsection{Actual trials: instructions}

Next, you will complete the actual trials for this experiment. You will complete three blocks (each consisting of 100 trials) in a random order:

1. You will complete the task manually without the decision aid.

2. You will complete the task using the decision aid. The final conclusion will be $80 \%$ reliable, while A1's and A2's conclusions will each be approximately $72 \%$ reliable.

3. You will complete the task using the decision aid. The final conclusion will be $94 \%$ reliable, while A1's and A2's conclusions will each be approximately $87 \%$ reliable.

- You will not be told if your answers are correct or incorrect.

- Answer each trial as quickly as possible without sacrificing accuracy. Each trial will be timed.

Similar to the practice trials:

- In every block, half the trials contain a high $X$ value, while the other half contains a normal $X$ value.

- Weigh right and wrong answers equally - correctly identifying the state of X is as important as avoiding a mistake.

Finally:

- Since the trials are timed, please concentrate on the trials: don't eat, use your cell phone, or do other work while performing the trials. You will have the chance to take breaks between blocks.

- Please read the instruction pop-up message carefully. 


\section{Appendix E: Training Comprehension Quiz}

1. What is your task for each trial?

a. Determine if the decision aid is correct

b. Determine if $\mathrm{X}$ is high or normal

c. Determine how reliable the decision aid is

2. What information should you use to make your decision?
a. The final confidence values
b. The A1 and A2 confidence values
c. The four estimates of $\mathrm{X}$
d. All of the above

3. For each block, what percentage of trials has a high $\mathrm{X}$ value?
a. $25 \%$ of trials
b. $50 \%$ of trials
c. $60 \%$ of trials
d. $75 \%$ of trials

4. Are the decision aid's results calculated using the 4 estimates given to you?
a. Yes
b. No
c. It depends on the trial
d. It depends on the block

5. If the decision aid's final result is $70 \%$ confident that $\mathrm{X}$ is high, what is its final confidence that $\mathrm{X}$ is normal?
a. $30 \%$
b. $70 \%$
c. $50 \%$
d. Cannot be calculated

6. What does the following statement mean? "A2 is $60 \%$ reliable."

a. A2's conclusion is correct in $60 \%$ of trials.

b. A2's conclusion is correct in $60 \%$ of trials when $\mathrm{X}$ is high.

c. A2's conclusion is correct in $60 \%$ of trials when $\mathrm{X}$ is normal. 
7. Is the final conclusion more reliable than the individual A1 and A2 conclusions?
a. The final conclusion is always more reliable
b. The final conclusion is always less reliable
c. It depends on the trial
d. It depends on the block

8. If $\mathrm{A} 1$ is $25 \%$ confident that $\mathrm{X}$ is high, what should the marker look like on the following confidence meter?

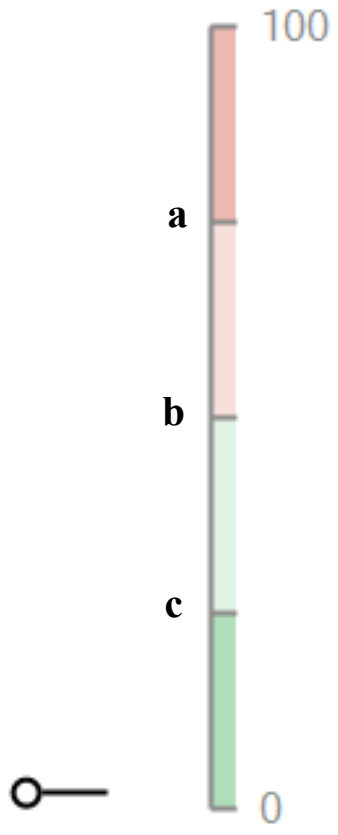
a. Green and at point ' $a$ '
b. Green and at point ' $b$ '
c. Green and at point ' $c$ '
d. Red and at point ' $a$ '
e. Red and at point ' $b$ '
f. Red and at point 'c'

9. Which is most important?

a. Correctly identifying when $\mathrm{X}$ is high

b. Correctly identifying when $\mathrm{X}$ is normal

c. Avoiding the error of responding " $\mathrm{X}$ is high" when $\mathrm{X}$ is actually normal

d. Avoiding the error of responding " $\mathrm{X}$ is normal" when $\mathrm{X}$ is actually high

e. All are equally important 


\section{Appendix F: Trust Questionnaire}

For each of the following 12 statements, please mark an "X" on each line at the point that best describes your feelings about the decision aid for the block (100 trials) you just completed.

$1=$ not at all;

$7=$ extremely

1. The decision aid is deceptive:

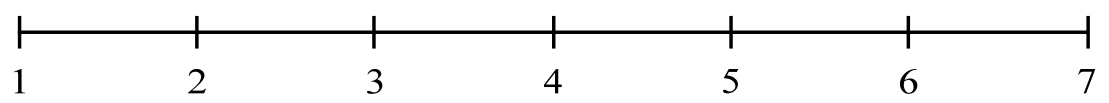

2. The decision aid behaves in an underhanded manner:

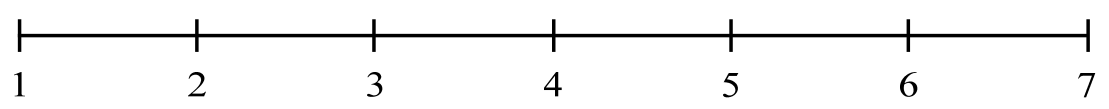

3. I am suspicious of the decision aid's intent, action, or outputs:

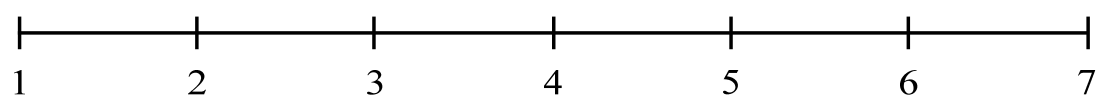

4. I am wary of the decision aid:

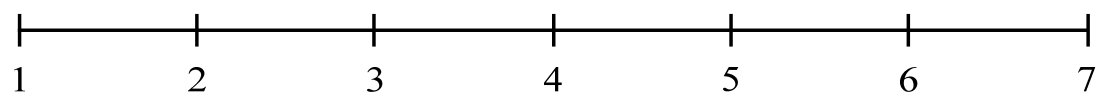

5. The decision aid's actions will have a harmful or injurious outcome:

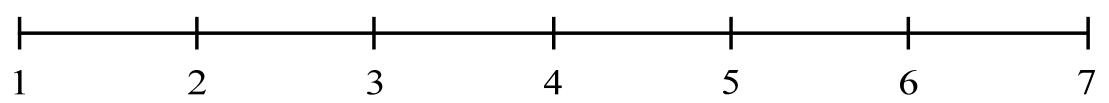

\section{Continued on back $\rightarrow$}


$1=$ not at all;

$7=$ extremely

6. I am confident in the decision aid:

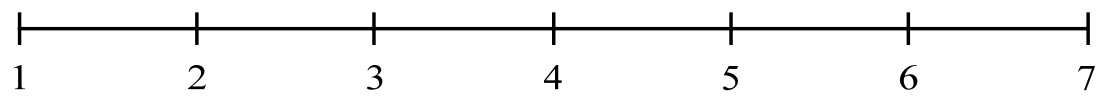

7. The decision aid provides security:

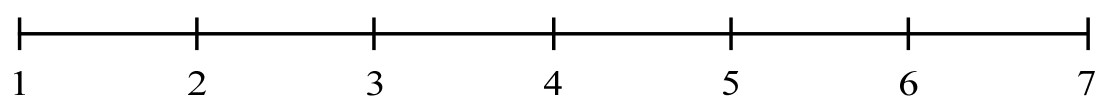

8. The decision aid has integrity:

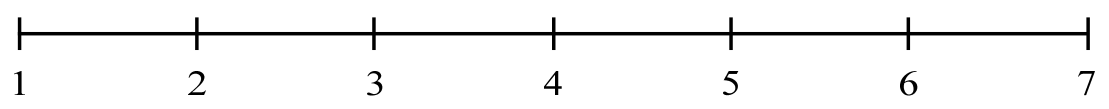

9. The decision aid is dependable:

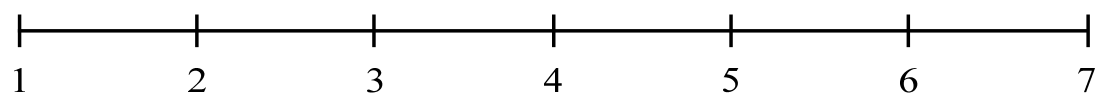

10. The decision aid is reliable:

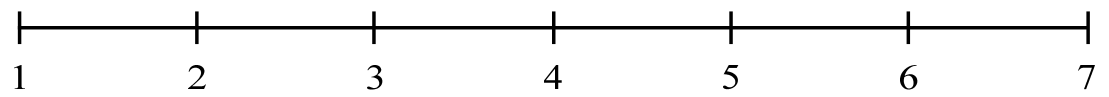

11. I can trust the decision aid:

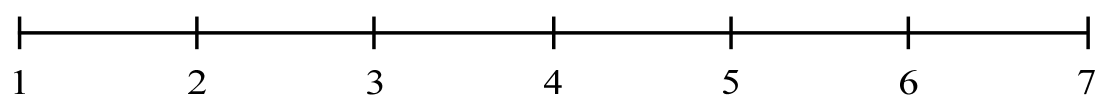

12. I am familiar with the decision aid:

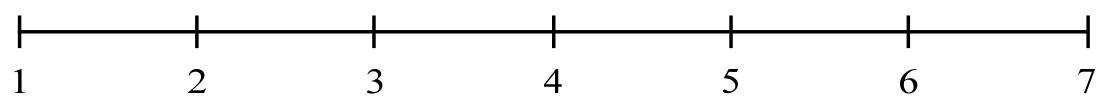

